\title{
Stay connected
}

Citation for published version (APA):

Domen, P. A. E. (2017). Stay connected: a family-based diffusion imaging study in psychotic disorder.

[Doctoral Thesis, Maastricht University]. Maastricht University. https://doi.org/10.26481/dis.20170412pd

\section{Document status and date:}

Published: 01/01/2017

DOI:

10.26481/dis.20170412pd

Document Version:

Publisher's PDF, also known as Version of record

\section{Please check the document version of this publication:}

- A submitted manuscript is the version of the article upon submission and before peer-review. There can be important differences between the submitted version and the official published version of record.

People interested in the research are advised to contact the author for the final version of the publication, or visit the DOI to the publisher's website.

- The final author version and the galley proof are versions of the publication after peer review.

- The final published version features the final layout of the paper including the volume, issue and page numbers.

Link to publication

\footnotetext{
General rights rights.

- You may freely distribute the URL identifying the publication in the public portal. please follow below link for the End User Agreement:

www.umlib.nl/taverne-license

Take down policy

If you believe that this document breaches copyright please contact us at:

repository@maastrichtuniversity.nl

providing details and we will investigate your claim.
}

Copyright and moral rights for the publications made accessible in the public portal are retained by the authors and/or other copyright owners and it is a condition of accessing publications that users recognise and abide by the legal requirements associated with these

- Users may download and print one copy of any publication from the public portal for the purpose of private study or research.

- You may not further distribute the material or use it for any profit-making activity or commercial gain

If the publication is distributed under the terms of Article $25 \mathrm{fa}$ of the Dutch Copyright Act, indicated by the "Taverne" license above, 


\section{Stay connected:}

A family-based diffusion imaging study in psychotic disorder 
Layout and Printing Ridderprint BV - www.ridderprint.nl Cover design G. Henderix - Hendrickx

ISBN 978-94-6299-572-7

(C) 2017 by Patrick Domen, Maastricht, the Netherlands

All rights reserved. No part of this publication may be reproduced or used in any manner whatsoever without prior written permission from the author. 


\title{
Stay connected:
}

\section{A family-based diffusion imaging study in psychotic disorder}

\author{
PROEFSCHRIFT
}

Ter verkrijging van de graad van doctor aan de Universiteit Maastricht, op gezag van de Rector Magnificus, Prof. dr. Rianne M. Letschert, volgens het besluit van het College van Decanen, in het openbaar te verdedigen op woensdag 12 april 2017 om 10:00 uur

door

Patrick Adrianus Elisabeth Domen 


\section{Promotor}

Prof. dr. J.J. van Os

\section{Copromotor}

Dr. M.C. Marcelis

\section{Beoordelingscommissie}

Prof. dr. Ph. Delespaul (voorzitter)

Prof. dr. T. van Amelsvoort

Dr. H. Knegtering (Universitair Medisch Centrum Groningen)

Dr. V. van de Ven

Prof. dr. R. Van Winkel (Katholieke Universiteit Leuven)

The research presented in this thesis was performed at the Department of Psychiatry and Neuropsychology, School for Mental Health and Neuroscience, Maastricht University, Maastricht, the Netherlands.

The research described in chapter 2-4 was funded by the Dutch organization for scientific research NWO (Genetic Risk and Outcome of Psychosis [G.R.O.U.P]) and the European Community's Seventh Framework Programme under Grant Agreement No. HEALTH-F2-2009-241909 (European Network of National Schizophrenia Networks Studying Gene-Environment Interactions Consortium). Both funding sources were not involved in the content of this thesis.

Publication of this thesis was financially supported by:

Lunbeck B.V.

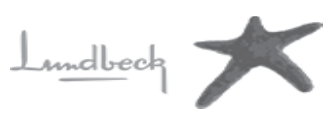


"Keep up with the force, don't stop. Don't stop 'til you get enough." (Michael J. Jackson) 


\section{Paranimfen}

Stijn Michielse

Jan Willem Plug 


\section{Contents}

$\begin{array}{lll}\text { Chapter } 1 & \text { General introduction } & 9\end{array}$

Chapter 2 Microstructural white matter alterations in psychotic disorder: 29

A family-based diffusion tensor imaging study

Chapter 3 Differential time-course of microstructural white matter in

patients with psychotic disorder and individuals at risk: $A$

3-year follow-up study

Chapter 4 Trauma- and cannabis-associated microstructural white matter changes in patients with psychotic disorder: A longitudinal family-based diffusion imaging study

Chapter 5 Discussion 109

Chapter 6 Epilogue

Summary 133

Samenvatting 137

Valorisation 143

Dankwoord 151

Curriculum Vitae 155

List of Publications 159 



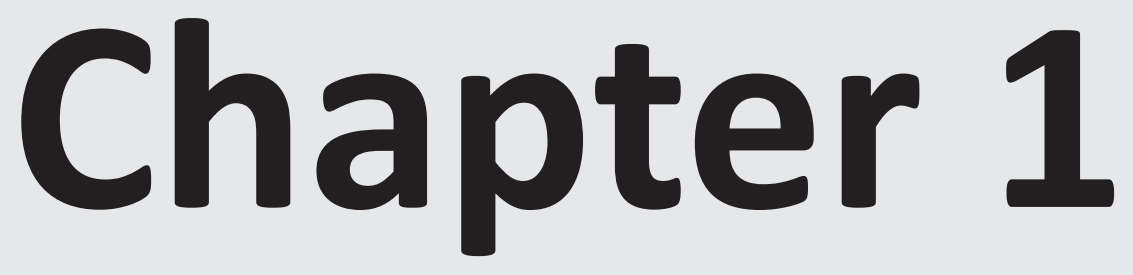

General introduction 



\section{Psychotic disorder}

The terms psychotic disorder or schizophrenia are often used interchangeably, whereas the latter is generally regarded as the more severe outcome on a psychosis continuum, with a lifetime prevalence of around $0.5-1.0 \%$ in the general population. Psychotic symptoms may occur in episodes or follow a more semi-continuous course and comprise hallucinations and delusions, frequently accompanied by affective, behavioural (avolition) and cognitive symptoms ${ }^{1}$. Symptoms need to be treated expeditiously, since a longer duration of untreated psychosis (DUP) is significantly associated with a worse symptomatic outcome, a lower risk to achieve complete remission, and a decrease in social functioning ${ }^{2}$. If diagnosed in time and treated with antipsychotic medication, symptomatic remission rates are $50-78 \%$. Importantly, nonpharmacological therapies play a central role in the treatment of psychotic disorder. Recovery (including good social and work functioning) rates still lag behind ${ }^{3}$, and stress the importance of non-pharmacological ingredients even more.

\section{The psychosis continuum}

Psychotic experiences are a common phenomenon ranging from subclinical symptoms in the general population (especially in young adults, fluctuating in time and severity, rarely inducing nuisance) with a lifetime prevalence of $10-20 \%$, to clinically disturbing symptoms or complaints (lifetime prevalence of $2-3 \%)^{4}$. Most of the brief or minor (pre)psychotic symptoms in adolescence never transgress to the clinical disorder and disappear without a need for care ${ }^{5}$. The prediction about who will make the conversion to psychosis is a challenge, as the prodromal phase appears to be rather non-specific, with a broader spectrum of symptoms, including anxiety and depressive symptoms ${ }^{6}$. For this purpose, the so-called at-risk-mental state (ARMS) or ultra-high risk (UHR) criteria were defined as either: $i)$ the presence of attenuated (subthreshold) psychotic symptoms, ii) a history of brief self limited psychotic symptoms, or iii) presumed genetic vulnerability to psychosis, in combination with persistent low functioning ${ }^{7}$. Recent studies have shown 12 -month transition rates of $10-15 \%$, and around $76 \%$ of the UHR subjects will not experience a psychotic episode during follow-up ${ }^{8,9}$. Once a psychotic episode has emerged, a proportion of individuals with the broader psychotic phenotype will attain good clinical and functional outcome, whereas the proportion of patients with the more narrowly defined phenotype (schizophrenia) who meet the recovery criteria, is only $13.5 \%$ and has not improved over the years

${ }^{10}$. Self-stigma, inducing lowered self-esteem and self-efficacy as well as social stigma, causing reduced access to employment and depleted social networks, may hamper recovery and functional remission ${ }^{11}$. 


\section{Etiology}

\section{Genetic risk factors}

The exact pathophysiology for schizophrenia is still unknown. Genetic contribution for the individual's risk for schizophrenia is estimated to be high, around $64-81 \%$. Candidate genes that have been described include the neuregulin1-tyrosine kinase receptor ErbB4 (NRG1-ErbB4), involved in the cortico-cortical myelination process during neurodevelopment ${ }^{13}$, the brain-derived neurotropic factor (BDNF) Val66Met variant, implicated in neuronal growth and brain plasticity, and the Catechol-O-methyl transferase (COMT) Val158met single nucleotide polymorphism, which has been associated with the inactivation of extra-neuronal dopamine ${ }^{14}$. A recent multi-stage genome-wide association study (GWAS) for schizophrenia identified 108 loci that met genome-wide significance. Associations at DRD2 and genes involved in glutamatergic neurotransmission comply with the leading pathophysiological (dopamine, glutamate) hypotheses ${ }^{12}$. Indeed, the dopamine hypothesis of psychosis is still the most prevailing one, suggesting that presynaptic striatal hyperdopaminergia is the final common pathway through which multiple environmental and genetic risk factors exert their effects. Within this theory, abnormal release of dopamine leads to an aberrant assignment of salience to otherwise neutral stimuli. This model may also explain the so-called negative symptoms of neglect and withdrawal, as this hyperdopaminergic state overrules dopaminergic reward signals to normal stimuli, resulting in reduced motivational drive ${ }^{15}$.

\section{Environmental risk factors}

The etiology of schizophrenia cannot be fully attributed to a genetic cause. Twin studies have shown that approximately $80 \%$ of the phenotypic variance may be explained by genetic variation ${ }^{16}$. Genetic risk variants may interact with environmental factors such as obstetric complications ${ }^{17,18}$, exposure to childhood trauma ${ }^{19}$, adolescent cannabis use ${ }^{20,21}$ and growing up in an urban environment ${ }^{22,23}$. Some of these risk factors are supposed to cause increased stress at a vulnerable age period (childhood, young adulthood) during the brains' most critical developmental phase. Repeated exposure to these stressors may lead to altered stress-signalling pathways resulting in disturbed neuronal responsiveness and symptoms of profound prefrontal cortical dysfunction ${ }^{24}$, as well as sensitization of mesolimbic dopamine neurons ${ }^{25}$, inducing psychotic symptoms. In addition, associations between childhood trauma and volume reductions in the corpus callosum ${ }^{26}$ and hippocampus ${ }^{27}$ have been reported, as well as impaired synaptic plasticity in relation to cannabis use ${ }^{28}$. In the above-mentioned studies, various neuroimaging techniques were used to link neuroanatomical abnormalities with psychological or biological models of psychotic disorder. 


\section{Structural brain imaging in psychotic disorder}

Magnetic resonance imaging (MRI) was introduced in the early 1980s as the successor of the X-ray computed tomography (CT). The CT scan was one of the earliest imaging techniques applied to psychiatric patients, providing the most replicated finding in patients with schizophrenia; enlargement of the lateral ventricles ${ }^{29}$. MRI research can be categorized in structural and functional neuroimaging. The focus in this thesis is primarily on structural imaging, as this is currently the best method for examining the effects of psychiatric disorders on brain volume and morphometry (size, shape) over time ${ }^{30}$. Since the first MRI study of schizophrenia in 1984 by Smith and colleagues (1984) ${ }^{31}$, numerous structural imaging results have been published. The main volumetric findings in patients with schizophrenia compared to healthy controls are: i) similar brain size, ii) enlarged lateral ventricles, iii) grey matter (GM) and white matter (WM) volume reductions in the (left) medial temporal lobe (hippocampus), the (left) superior temporal gyrus and less consistent abnormalities in the parietal lobe and subcortical regions (basal ganglia, cerebellum, thalamus and corpus callosum) ${ }^{32,33}$. Studies measuring cortical thickness, a proxy measure of cyto-architectural integrity, have found widespread cortical thinning in mainly fronto-temporo-parietal regions in patients with chronic ${ }^{34}$ and subacute ${ }^{35}$ psychotic disorder, as well as in patients with first-episode psychosis ${ }^{36}$ compared to healthy controls.

The exact timing of onset and course of the brain alterations in schizophrenia is unresolved. Schizophrenia has been described as a neurodevelopmental disorder with brain alterations caused by susceptibility genes and environmental factors occurring in early life before disease onset ${ }^{37}$, as a progressive or a neurodegenerative disorder with brain alterations emerging around disease onset and worsening over time ${ }^{38}$, and as a combination of both. Evidence for a neurodevelopmental origin have been obtained from studies reporting structural brain volume differences ${ }^{39}$, for instance in the anterior cingulate gyrus ${ }^{40}$, in clinical high risk samples before the onset of psychotic symptoms. Progressive brain alterations were found in a large systematic review and meta-analysis by Olabi and colleagues (2011), showing statistically significant progressive reductions in whole brain, whole brain GM and frontal lobe volumes, as well as frontal, parietal, and temporal lobe white matter decrements and lateral ventricular volume enlargement over time in patients with schizophrenia compared to healthy controls ${ }^{41}$. These results seem to support both models, suggesting that the cerebral alterations in psychosis involve an on-going process of genetic and environmental as well as epistatic and epigenetic interactions ${ }^{37,42}$, but also illness- or treatment related factors. As stated before, multiple brain areas have been associated with schizophrenia, pointing to altered networks / neuronal connectivity, as demonstrated in several neurophysiological and functional imaging studies ${ }^{43,44}$. The dyscon- 
nectivity hypothesis as formulated by Friston (1998) implies that the core pathology of schizophrenia is the result of faults in abnormal epigenetic, activity- and experience dependent $\mathrm{N}$-methyl-D-aspartate receptor (NMDAR) mediated synaptic plasticity by modulatory neurotransmitters like dopamine. This aberrant wiring may cause longterm changes in connection strength of functionally specialized interconnected brain systems, leading to a failure in self-monitoring and eventually the characteristic psychotic symptoms ${ }^{45,46}$. A structural imaging modality named diffusion tensor imaging (DTI) and functional MRI (fMRI) are commonly used to study respectively structural and functional inter-regional connectivity.

\section{Diffusion tensor imaging}

For the last twenty years, DTI has been used to investigate neurological disorders, especially in patients with acute stroke. As an indirect measure for the connectivity of white matter axons in the central nervous system, it has also rapidly become a standard for disorders that are associated with white matter abnormalities. DTI uses the principle of molecular diffusion, a random translational motion of molecules (also called Brownian motion or isotropic diffusion, if not hindered by any boundaries). However, in the brain diffusion patterns are different, as water molecules bounce, cross and interact with various tissue components as cell membranes, and (myelinated) axonal fibres. Water molecules tend to move along the axis of the neuron, parallel to the direction of the fibres, which is called anisotropic diffusion. This diffusion data can be analysed for information on tissue microstructure and fibre architecture, using the diffusion tensor in three different ways and calculating the: i) mean diffusivity, ii) fractional anisotropy (FA), relating to the presence and coherence of oriented structures and iii) main direction of diffusivities ${ }^{47,48}$. In this thesis, FA will be the most discussed diffusion coefficient, set out between 0 and 1 ; high in white matter, low in grey matter and close to zero in cerebrospinal fluid. Regional FA changes may point to white matter abnormalities, but do not specify the nature of the change. WM alterations may be multi-causal, referring to a distorted demyelination process, fibre reduction or incoherence, or differences in the proportion of axonal, oligodendrocyte and glial cellular components ${ }^{49}$. In addition, FA is not a steady value, but follows a tract-specific curvilinear maturation pattern, with an increase of FA from newborn to adolescence, decelerated maturation until mid-adulthood and then a gradual decline at old age ${ }^{50}$. 


\section{Diffusion studies in psychotic disorder}

\section{Patient - control differences}

Over three hundred diffusion studies have been performed since the late 1990s with patients with a psychotic disorder in various stages of the illness. Numerous crosssectional studies reported widespread white matter alterations in patient samples compared to healthy controls, whereby the findings in first-episode patients seem less robust than in later stage patients. Several WM regions seem to be affected, whereas the most frequently studied and mentioned major WM tract is the corpus callosum, followed by the arcuate fasciculus, fronto-occipital fasciculus, but also more broader defined frontal, temporal, and parietal regions ${ }^{51}$. A meta-analysis by Ellison-Wright \& Bullmore (2009), based on 15 studies, showed two regions with significant FA reductions: one cluster in the left frontal deep white matter (anterior thalamic radiation and corticobulbar tracts, inter-hemispheric fibres running through the genu of the corpus callosum, the inferior fronto-occipital fasciculus and the cingulum bundle) and the left temporal deep white matter (interhemispheric fibers running through the splenium of the corpus callosum, the inferior fronto-occipital fasciculus, the inferior longitudinal fasciculus and the fornix/stria terminalis) ${ }^{52}$. Differences in methodology and sample characteristics (e.g. clinical heterogeneity, smoking history, exercise, body weight, lipid levels) as well as systematic confounders (motion artefacts, medication) may explain the varying patterns between studies ${ }^{53}$.

Overall, psychotic disorder has been related to widespread FA decreases in the literature. In contrast, some studies reported FA increases in fibres connecting temporal regions (such as in the left medial temporal lobe) in clinical high risk subjects who made the transition to psychosis compared to the non-transition group ${ }^{54}$. The same applies to the arcuate fasciculus ${ }^{55,56}$ and superior longitudinal fasciculus ${ }^{57}$, in patients with psychotic disorder experiencing more severe hallucinations.

Notwithstanding the fact that associations between WM alterations and clinical symptoms have yielded inconclusive results (showing significant associations in half of the studies), positive associations between FA and positive symptoms (e.g. inferior longitudinal fasciculus, cingulum bundle) and negative associations between FA and negative symptoms (uncinate fasciculus, superior longitudinal fasciculus) have been reported $^{58}$.

\section{Antipsychotic medication}

The potential influence of illness-related factors in patients with psychotic disorder, such as a disadvantageous life style and health issues (e.g. lack of exercise, social deprivation, smoking), on brain tissue is not well understood. The same applies to the potential effect of antipsychotic (AP) medication on brain structure ${ }^{59}$. Here, it 
has to be kept in mind that accounting for AP use in imaging studies with a patient - control design is complex, as it may imply controlling for patient-status. In recent years, increasing attention has been given to the examination of AP effects on WM integrity in cross-sectional and longitudinal studies with contrasting results. A negative (trend) significant association between FA and duration of AP use in certain brain regions (arcuate fasciculus, superior longitudinal fasciculus) ${ }^{58}$, FA reductions within the parietal and occipital WM after 12 weeks of AP treatment ${ }^{60}$ as well as FA increases in widespread brain regions after 12 weeks op clozapine treatment ${ }^{61}$.

As microstructural WM alterations can also be detected in medication-naïve patients 62,63 , AP use is most likely one of the contributing factors to brain alteration. Besides the clinical high-risk approach, another way to contribute to the medication debate is to investigate WM structure in individuals with a higher-than-average genetic risk for psychosis (e.g. unaffected relatives of patients with psychotic disorder).

\section{Intermediate phenotype}

Examining brain alterations in unaffected relatives of patients with a psychotic disorder is also a precondition for identifying a diffusion intermediate phenotype. An intermediate phenotype is a measurable biological marker, in this case a microstructural WM alteration, related to the underlying disease mechanism with a genetic foundation. A candidate intermediate phenotype should at least e.g. be heritable, be present in absence of certain symptoms and be present in unaffected relatives of patients at a higher rate than the general population ${ }^{64}$. A recent qualitative review by Arat and colleagues (2015) provided an overview of 13 studies measuring diffusion parameters in unaffected siblings of patients with schizophrenia compared to healthy controls. Although based on a relatively small number of studies with varying methodological and sample characteristics, it was concluded that most studies show WM abnormalities in unaffected siblings in similar regions (e.g. frontal and temporal WM tracts) as identified in patients with a psychotic disorder. Especially in the genu of the corpus callosum significant differences in diffusion parameters were found ${ }^{65,66}$, leading the authors to state that disrupted WM integrity in this region may be an intermediate phenotype for schizophrenia ${ }^{67}$.

\section{FA course over time}

Conform the volumetric findings, neurodevelopmental decreased WM integrity in clinical high-risk populations ${ }^{68,69}$, as well as post-onset progression of widespread WM alterations in patients with late stage psychotic disorder ${ }^{70}$ has been reported. Longitudinal diffusion studies are scarce and have focused on changes in WM integrity during transition from UHR status to first-episode psychosis status ${ }^{71}$, or before and after short periods of AP treatment in first-episode ${ }^{72}$ and late stage patients ${ }^{73}$. 
Only one study examined the course of WM development over a longer period of time in patients with chronic schizophrenia $(n=49)$ and found a stronger FA decline over 4 years in patients compared to controls in the left anterior cingulate and in the bilateral orbitofrontal region ${ }^{74}$, suggesting a differential WM integrity course over time. Clearly, there is a gap in the literature with regard to longitudinal studies of microstructural WM alterations over the course of illness.

\section{Genes, environment and microstructural white matter}

WM volume in healthy twin-samples has shown to be a highly heritable phenotype. It is estimated that genetic factors account for around $85-88 \%$ of the individual difference in whole brain WM volume ${ }^{75,76}$. White matter FA shows moderate to high heritability $(0.4-0.7)$ depending on the tract, and with the exception of the corticospinal tract (lower values). The heritability indices are slightly higher during neonatal period and childhood ${ }^{77,78}$, but on the whole seem rather stable. Recently, Bohlken and colleagues (2016) reported that $83.4 \%$ of the association between global FA and schizophrenia liability can be explained by common genes ${ }^{79}$. In other words, the white matter alterations can, to a great extent, be seen as a genetic liability to schizophrenia.

Candidate genes for WM heritability (in health and mental disorder) that have been reported indeed are involved in oligodendrocyte, myelin and axonal development and maintenance. Over the years, the neuregulin1-tyrosine kinase receptor ErbB4 (NRG1ErbB4) gene system (because of its apparent role in cortico-cortical myelination process during neurodevelopment ${ }^{80}$ ), the disrupted-in-schizophrenia 1 (DISC1) gene ${ }^{81}$, and a val66met BDNF polymorphism ${ }^{82}$ have been mentioned in this respect. In more recent imaging-genetic studies, the focus has shifted to the GWAS risk genes for schizophrenia and their impact on brain structure. For example, associations between white matter integrity and ANK3 and ZNF804A ${ }^{83}$ were reported. In healthy young adults, a NTRK3 gene variant associated with WM integrity of the corpus callosum has been described ${ }^{84}$.

In contrast to the ample number of genetic studies on the etiology of WM pathology in psychotic disorder, studies examining and integrating environmental risk factors in a design are scarce. There are some studies examining the effect of cannabis exposure on WM structure in patients with schizophrenia, showing FA increases as well as decreases in cannabis using versus non-using patients ${ }^{51,85}$. The effect of childhood trauma exposure on microstructural WM development has only been investigated in non-psychotic samples. For example, reduced FA in the inferior longitudinal fasciculus of the left lateral occipital lobe in twenty young adults witnessing domestic violence in childhood ${ }^{86}$, and reduced FA in the medial and posterior corpus callosum in traumatized children with posttraumatic stress disorder ${ }^{87}$ have been reported. Except for a 
few volumetric studies, there has been no investigation on associations of obstetrical complications and WM integrity and developmental urbanicity and WM integrity. A study by Cannon and colleagues (2002) found that foetal hypoxia was not related to WM volume, but predicted reduced GM volume and increased CSF bilaterally throughout the cortex, especially in the temporal lobe, in patients with schizophrenia and their unaffected siblings compared to healthy controls ${ }^{88}$. A voxel-based morphometry study reported an inverse correlation between early-life urbanicity and GM volume in the right dorsolateral prefrontal cortex and a negative correlation with the perigenual anterior cingulate cortex GM volume in healthy males ${ }^{89}$. Thus, there is room for integrating the environment/context in diffusion studies in order to better understand cerebral microstructural white matter alterations in the psychosis syndrome.

\section{Outline and aim of the thesis}

The aim of this thesis is to examine whole-brain white matter integrity, cross-sectionally as well as a longitudinally, in patients with a psychotic disorder with a relatively short-moderate illness duration, in individuals at higher-than-average genetic risk (unaffected siblings of patients with a psychotic disorder) and in individuals at average genetic risk (healthy controls). Therefore, diffusion tensor imaging scans were collected to: i) determine whether a microstructural WM endophenotype for psychotic disorder can be identified, ii) investigate the pattern/course of microstructural WM connectivity alterations over time, iii) examine whether microstructural WM connectivity alterations can be appraised as the outcome of differential sensitivity to environmental risk factors (i.e. cannabis use and childhood trauma).

\section{Study design}

Participants were recruited in the context of a large multi-site (the academic psychiatric centres of Amsterdam, Groningen, Maastricht, Utrecht and their affiliated mental health care institutions) longitudinal, observational, cohort study: Genetic Risk and Outcome of Psychosis (G.R.O.U.P.) in the Netherlands. This study was held from April 2004 till December 2013, examining the vulnerability and protective factors for psychotic disorder by assessing genetic, biological, psychological and social factors. During the baseline measurement, data of 1120 patients with a non-affective psychotic disorder, 1057 of their healthy siblings, 919 of their parents and 590 unrelated healthy controls have been collected, including symptomatology measures, various risk and protective factors, blood and urine samples (DNA analysis and drug screenings) ${ }^{90}$. In addition, structural (including diffusion imaging) and functional MRI scans were obtained locally at Maastricht University using a 3T Siemens scanner. Participants from 
a selected representative geographical area around Maastricht and in Belgium were included. At baseline, the total MRI sample comprised 274 participants: 89 patients with a psychotic disorder, 98 unaffected siblings and 87 healthy controls. At follow up (on average three years later), roughly two-third of this sample was scanned for a second time. Studies described in this thesis were conducted with this G.R.O.U.P. MRI add-on sample.

\section{Thesis content}

The search for a WM intermediate phenotype is discussed in chapter 2. To date, numerous DTI studies in patients with psychotic disorder have undisputedly shown a decrease in FA in fronto-temporal and fronto-occipital connections, indicative of white matter integrity loss. Fewer DTI studies, with smaller sample sizes, have been performed on individuals at higher-than-average genetic risk for psychotic disorder. Nonetheless, the available evidence suggests that white matter alterations may also be present in first-degree relatives without psychotic symptoms, pointing to a possible WM intermediate phenotype. Group differences in white matter integrity were analysed, using a whole-brain DTI approach to examine patients with a psychotic disorder, their non-psychotic siblings, and a healthy control group $(n=258)$. Based on previous literature, we hypothesized that patients with psychotic disorder would show reduced white matter integrity compared to healthy controls, particularly in the corpus callosum, fronto-temporal, and fronto-parietal connections, with siblings showing a intermediate pattern of alterations. Confounding by medication or illicit drugs and sex differences were investigated as part of the main analyses.

Chapter $\mathbf{3}$ addresses the topic of white matter progression over time in patients with a psychotic disorder as well as their healthy siblings. Evidence for WM alterations in clinical high-risk populations and first episode patients derive mainly from crosssectional DTI studies. Longitudinal diffusion studies are scarce and, as a consequence, the clinical evidence for post-onset microstructural with matter progression is minimal. The aim of this study was to investigate longitudinally the course of microstructural WM change over time in a family study design. Individuals were scanned twice $(n=159)$ with an average of a 3-year interval. We hypothesized that in individuals with (liability for) psychosis, whole-brain FA decrease would be stronger and earlier than expected by age as compared to controls, indicating differential trajectories over time as a function of level of psychosis liability.

The same longitudinal design was used to examine the impact of environmental risk factors of psychotic disorder on white matter alterations. That study is presented in chapter 4. Although epidemiological studies have pointed to environmental exposures such as pre- or postnatal birth complications, cannabis use, developmental trauma and urbanicity as risk factors for psychotic disorder, their association with cerebral 
alteration has hardly been studied. In this study we investigated whether microstructural white matter alterations in individuals with (liability for) psychotic disorder were conditional on the exposure to cannabis or childhood trauma. More specifically, we hypothesized that individuals at increased genetic risk (patients and siblings) with higher exposure levels to cannabis and childhood trauma would show reduced white matter integrity at baseline and increased WM deterioration over time.

In chapter 5, the findings of this thesis are integrated and discussed in more detail, providing directions for future research. 


\section{References}

1. van Os J, Kenis G, Rutten BP. The environment and schizophrenia. Nature Nov 11 2010;468(7321):203-212.

2. Penttila $M$, Jaaskelainen $E$, Hirvonen $N$, Isohanni $M$, Miettunen J. Duration of untreated psychosis as predictor of long-term outcome in schizophrenia: systematic review and metaanalysis. The British journal of psychiatry : the journal of mental science Aug 2014;205(2):8894.

3. Ventura J, Subotnik KL, Guzik LH, Hellemann GS, Gitlin MJ, Wood RC, Nuechterlein KH. Remission and recovery during the first outpatient year of the early course of schizophrenia. Schizophrenia research Oct 2011;132(1):18-23.

4. van Os J, Linscott RJ, Myin-Germeys I, Delespaul P, Krabbendam L. A systematic review and meta-analysis of the psychosis continuum: evidence for a psychosis proneness-persistenceimpairment model of psychotic disorder. Psychological medicine Feb 2009;39(2):179-195.

5. Kaymaz N, Drukker M, Lieb R, Wittchen HU, Werbeloff N, Weiser M, Lataster T, van Os J. Do subthreshold psychotic experiences predict clinical outcomes in unselected non-help-seeking population-based samples? A systematic review and meta-analysis, enriched with new results. Psychological medicine Nov 2012;42(11):2239-2253.

6. Buckley PF, Miller BJ, Lehrer DS, Castle DJ. Psychiatric comorbidities and schizophrenia. Schizophrenia bulletin Mar 2009;35(2):383-402.

7. Yung AR, Stanford C, Cosgrave E, Killackey E, Phillips L, Nelson B, McGorry PD. Testing the Ultra High Risk (prodromal) criteria for the prediction of psychosis in a clinical sample of young people. Schizophrenia research May 2006;84(1):57-66.

8. Fusar-Poli P, Bonoldi I, Yung AR, et al. Predicting psychosis: meta-analysis of transition outcomes in individuals at high clinical risk. Archives of general psychiatry Mar 2012;69(3):220-229.

9. Simon AE, Velthorst E, Nieman DH, Linszen D, Umbricht D, de Haan L. Ultra high-risk state for psychosis and non-transition: a systematic review. Schizophrenia research Oct 2011;132(1):817.

10. Jaaskelainen E, Juola P, Hirvonen N, McGrath JJ, Saha S, Isohanni M, Veijola J, Miettunen J. A systematic review and meta-analysis of recovery in schizophrenia. Schizophrenia bulletin Nov 2013;39(6):1296-1306.

11. Karidi MV, Stefanis CN, Theleritis C, Tzedaki M, Rabavilas AD, Stefanis NC. Perceived social stigma, self-concept, and self-stigmatization of patient with schizophrenia. Comprehensive psychiatry Jan-Feb 2010;51(1):19-30.

12. Ripke $\mathrm{S}$, et al. Biological insights from 108 schizophrenia-associated genetic loci. Nature 2014;511 (7510):421-427.

13. Norton N, Moskvina V, Morris DW, et al. Evidence that interaction between neuregulin 1 and its receptor erbB4 increases susceptibility to schizophrenia. American journal of medical genetics Part B, Neuropsychiatric genetics : the official publication of the International Society of Psychiatric Genetics Jan 5 2006;141B(1):96-101.

14. Gatt JM, Burton KL, Williams LM, Schofield PR. Specific and common genes implicated across major mental disorders: A review of meta-analysis studies. Journal of psychiatric research Jan 2015;60C:1-13.

15. Howes OD, Kapur S. The dopamine hypothesis of schizophrenia: version III--the final common pathway. Schizophrenia bulletin May 2009;35(3):549-562. 
16. Sullivan PF, Kendler KS, Neale MC. Schizophrenia as a complex trait: evidence from a metaanalysis of twin studies. Archives of general psychiatry Dec 2003;60(12):1187-1192.

17. Cannon M, Jones PB, Murray RM. Obstetric complications and schizophrenia: historical and meta-analytic review. Am J Psychiatry Jul 2002;159(7):1080-1092.

18. Marcelis M, van Os J, Sham P, Jones P, Gilvarry C, Cannon M, McKenzie K, Murray R. Obstetric complications and familial morbid risk of psychiatric disorders. Am J Med Genet Feb 7 1998;81(1):29-36.

19. Varese F, Smeets F, Drukker M, et al. Childhood Adversities Increase the Risk of Psychosis: A Meta-analysis of Patient-Control, Prospective- and Cross-sectional Cohort Studies. Schizophrenia bulletin Mar 29 2012:661-671.

20. Arseneault L, Cannon M, Witton J, Murray RM. Causal association between cannabis and psychosis: examination of the evidence. The British journal of psychiatry : the journal of mental science Feb 2004;184:110-117.

21. Henquet C, Krabbendam L, Spauwen J, Kaplan C, Lieb R, Wittchen HU, van Os J. Prospective cohort study of cannabis use, predisposition for psychosis, and psychotic symptoms in young people. Bmj Jan 1 2005;330(7481):11.

22. Marcelis M, Takei N, van Os J. Urbanization and risk for schizophrenia: does the effect operate before or around the time of illness onset? Psychological medicine Sep 1999;29(5):11971203.

23. Pedersen CB, Mortensen PB. Evidence of a dose-response relationship between urbanicity during upbringing and schizophrenia risk. Archives of general psychiatry Nov 2001;58(11):10391046.

24. Arnsten AF. Stress signalling pathways that impair prefrontal cortex structure and function. Nature reviews Neuroscience Jun 2009;10(6):410-422.

25. Lieberman JA, Sheitman BB, Kinon BJ. Neurochemical sensitization in the pathophysiology of schizophrenia: deficits and dysfunction in neuronal regulation and plasticity. Neuropsychopharmacology : official publication of the American College of Neuropsychopharmacology Oct 1997;17(4):205-229.

26. Teicher $\mathrm{MH}$, Dumont NL, Ito $\mathrm{Y}$, Vaituzis $\mathrm{C}$, Giedd JN, Andersen SL. Childhood neglect is associated with reduced corpus callosum area. Biol Psychiatry Jul 15 2004;56(2):80-85.

27. Andersen SL, Tomada A, Vincow ES, Valente E, Polcari A, Teicher MH. Preliminary evidence for sensitive periods in the effect of childhood sexual abuse on regional brain development. The Journal of neuropsychiatry and clinical neurosciences Summer 2008;20(3):292-301.

28. Robbe D, Alonso G, Manzoni OJ. Exogenous and endogenous cannabinoids control synaptic transmission in mice nucleus accumbens. Annals of the New York Academy of Sciences Nov 2003;1003:212-225.

29. Andreasen NC. Evaluation of brain imaging techniques in mental illness. Annual review of medicine 1988;39:335-345.

30. Gur RE, Keshavan MS, Lawrie SM. Deconstructing psychosis with human brain imaging. Schizophrenia bulletin Jul 2007;33(4):921-931.

31. Smith RC, Calderon M, Ravichandran GK, Largen J, Vroulis G, Shvartsburd A, Gordon J, Schoolar JC. Nuclear magnetic resonance in schizophrenia: a preliminary study. Psychiatry research Jun 1984;12(2):137-147.

32. Honea R, Crow TJ, Passingham D, Mackay CE. Regional deficits in brain volume in schizophrenia: a meta-analysis of voxel-based morphometry studies. Am J Psychiatry Dec 2005;162(12):2233-2245. 
33. Shenton ME, Dickey CC, Frumin M, McCarley RW. A review of MRI findings in schizophrenia. Schizophrenia research Apr 15 2001;49(1-2):1-52.

34. Nesvag R, Lawyer G, Varnas K, Fjell AM, Walhovd KB, Frigessi A, Jonsson EG, Agartz I. Regional thinning of the cerebral cortex in schizophrenia: effects of diagnosis, age and antipsychotic medication. Schizophrenia research Jan 2008;98(1-3):16-28.

35. Schultz CC, Koch K, Wagner G, et al. Complex pattern of cortical thinning in schizophrenia: results from an automated surface based analysis of cortical thickness. Psychiatry research May 30 2010;182(2):134-140.

36. Schultz CC, Koch K, Wagner G, et al. Reduced cortical thickness in first episode schizophrenia. Schizophrenia research Feb 2010;116(2-3):204-209.

37. Rapoport JL, Giedd JN, Gogtay N. Neurodevelopmental model of schizophrenia: update 2012. Molecular psychiatry Dec 2012;17(12):1228-1238.

38. Meyer-Lindenberg A. Neuroimaging and the question of neurodegeneration in schizophrenia. Progress in neurobiology Dec 2011;95(4):514-516.

39. Lawrie SM, Mclntosh AM, Hall J, Owens DG, Johnstone EC. Brain structure and function changes during the development of schizophrenia: the evidence from studies of subjects at increased genetic risk. Schizophrenia bulletin Mar 2008;34(2):330-340.

40. Fornito A, Yung AR, Wood SJ, et al. Anatomic abnormalities of the anterior cingulate cortex before psychosis onset: an MRI study of ultra-high-risk individuals. Biol Psychiatry Nov 1 2008;64(9):758-765.

41. Olabi B, Ellison-Wright I, McIntosh AM, Wood SJ, Bullmore E, Lawrie SM. Are there progressive brain changes in schizophrenia? A meta-analysis of structural magnetic resonance imaging studies. Biol Psychiatry Jul 1 2011;70(1):88-96.

42. Harrison PJ, Weinberger DR. Schizophrenia genes, gene expression, and neuropathology: on the matter of their convergence. Molecular psychiatry Jan 2005;10(1):40-68; image 45.

43. Andreasen NC, Paradiso S, O' Leary DS. "Cognitive dysmetria" as an integrative theory of schizophrenia; a dysfunction in cortical - subcortical - cerebellar circuitry. Schizophrenia bulletin 1998;24(2):203-218.

44. Friston KJ, Frith CD. Schizophrenia: a disconnection syndrome? Clin Neurosci 1995;3(2):89-97.

45. Friston KJ. The Disconnection hypothesis. Schizophrenia research 1998;30:115-125.

46. Stephan KE, Friston KJ, Frith CD. Dysconnection in schizophrenia: from abnormal synaptic plasticity to failures of self-monitoring. Schizophrenia bulletin May 2009;35(3):509-527.

47. Le Bihan D, Johansen-Berg H. Diffusion MRI at 25: exploring brain tissue structure and function. Neuroimage Jun 2012;61(2):324-341.

48. Le Bihan D, Poupon C, Amadon A, Lethimonnier F. Artifacts and pitfalls in diffusion MRI. J Magn Reson Imaging Sep 2006;24(3):478-488.

49. Assaf $Y$, Pasternak O. Diffusion tensor imaging (DTI)-based white matter mapping in brain research: a review. Journal of molecular neuroscience : MN 2008;34(1):51-61.

50. Imperati D, Colcombe S, Kelly C, Di Martino A, Zhou J, Castellanos FX, Milham MP. Differential development of human brain white matter tracts. PloS one 2011;6(8):e23437.

51. Peters BD, Blaas J, de Haan L. Diffusion tensor imaging in the early phase of schizophrenia: what have we learned? Journal of psychiatric research Nov 2010;44(15):993-1004.

52. Ellison-Wright I, Bullmore E. Meta-analysis of diffusion tensor imaging studies in schizophrenia. Schizophrenia research Mar 2009;108(1-3):3-10.

53. Weinberger DR, Radulescu E. Finding the Elusive Psychiatric "Lesion" With 21st-Century Neuroanatomy: A Note of Caution. Am J Psychiatry Jan 1 2016;173(1):27-33. 
54. Bloemen OJ, de Koning MB, Schmitz N, et al. White-matter markers for psychosis in a prospective ultra-high-risk cohort. Psychological medicine Aug 2010;40(8):1297-1304.

55. Hubl D, Koenig T, Strik W, et al. Pathways that make voices: white matter changes in auditory hallucinations. Archives of general psychiatry Jul 2004;61(7):658-668.

56. Rotarska-Jagiela A, Oertel-Knoechel V, DeMartino F, et al. Anatomical brain connectivity and positive symptoms of schizophrenia: a diffusion tensor imaging study. Psychiatry research Oct 30 2009;174(1):9-16

57. Shergill SS, Kanaan RA, Chitnis XA, et al. A diffusion tensor imaging study of fasciculi in schizophrenia. Am J Psychiatry Mar 2007;164(3):467-473.

58. Samartzis L, Dima D, Fusar-Poli P, Kyriakopoulos M. White matter alterations in early stages of schizophrenia: a systematic review of diffusion tensor imaging studies. Journal of neuroimaging : official journal of the American Society of Neuroimaging Mar-Apr 2014;24(2):101-110.

59. Navari S, Dazzan P. Do antipsychotic drugs affect brain structure? A systematic and critical review of MRI findings. Psychological medicine Nov 2009;39(11):1763-1777.

60. Szeszko PR, Robinson DG, Ikuta T, Peters BD, Gallego JA, Kane J, Malhotra AK. White matter changes associated with antipsychotic treatment in first-episode psychosis. Neuropsychopharmacology : official publication of the American College of Neuropsychopharmacology May 2014;39(6):1324-1331.

61. Ozcelik-Eroglu E, Ertugrul A, Oguz KK, Has AC, Karahan S, Yazici MK. Effect of clozapine on white matter integrity in patients with schizophrenia: a diffusion tensor imaging study. Psychiatry research Sep 30 2014;223(3):226-235.

62. Cheung V, Chiu CP, Law CW, et al. Positive symptoms and white matter microstructure in never-medicated first episode schizophrenia. Psychological medicine Aug 2011;41(8):17091719.

63. Gasparotti R, Valsecchi P, Carletti F, Galluzzo A, Liserre R, Cesana B, Sacchetti E. Reduced fractional anisotropy of corpus callosum in first-contact, antipsychotic drug-naive patients with schizophrenia. Schizophrenia research Mar 2009;108(1-3):41-48.

64. Gottesman, II, Gould TD. The endophenotype concept in psychiatry: etymology and strategic intentions. Am J Psychiatry Apr 2003;160(4):636-645.

65. Camchong J, Lim KO, Sponheim SR, Macdonald AW. Frontal white matter integrity as an endophenotype for schizophrenia: diffusion tensor imaging in monozygotic twins and patients' nonpsychotic relatives. Front Hum Neurosci 2009;3:35.

66. Knochel C, Oertel-Knochel V, Schonmeyer R, et al. Interhemispheric hypoconnectivity in schizophrenia: Fiber integrity and volume differences of the corpus callosum in patients and unaffected relatives. Neurolmage Jan 16 2012;59(2):926-934.

67. Arat HE, Chouinard VA, Cohen BM, Lewandowski KE, Ongur D. Diffusion tensor imaging in first degree relatives of schizophrenia and bipolar disorder patients. Schizophrenia research Feb 2015;161(2-3):329-339.

68. Clemm von Hohenberg C, Pasternak O, Kubicki M, et al. White Matter Microstructure in Individuals at Clinical High Risk of Psychosis: A Whole-Brain Diffusion Tensor Imaging Study. Schizophrenia bulletin 2014 Jul;40(4):895-903.

69. Karlsgodt KH, Niendam TA, Bearden CE, Cannon TD. White matter integrity and prediction of social and role functioning in subjects at ultra-high risk for psychosis. Biol Psychiatry Sep 15 2009;66(6):562-569. 
70. Mori T, Ohnishi T, Hashimoto R, et al. Progressive changes of white matter integrity in schizophrenia revealed by diffusion tensor imaging. Psychiatry research Feb 28 2007;154(2):133145.

71. Carletti F, Woolley JB, Bhattacharyya S, et al. Alterations in white matter evident before the onset of psychosis. Schizophrenia bulletin Nov 2012;38(6):1170-1179.

72. Reis Marques $\mathrm{T}$, Taylor $\mathrm{H}$, Chaddock $\mathrm{C}$, et al. White matter integrity as a predictor of response to treatment in first episode psychosis. Brain : a journal of neurology Jan 2014;137(Pt 1):172182.

73. Garver DL, Holcomb JA, Christensen JD. Compromised myelin integrity during psychosis with repair during remission in drug-responding schizophrenia. The international journal of neuropsychopharmacology / official scientific journal of the Collegium Internationale Neuropsychopharmacologicum Feb 2008;11(1):49-61.

74. Mitelman SA, Canfield EL, Newmark RE, et al. Longitudinal Assessment of Gray and White Matter in Chronic Schizophrenia: A Combined Diffusion-Tensor and Structural Magnetic Resonance Imaging Study. The open neuroimaging journal 2009;3:31-47.

75. Baare WF, Hulshoff Pol HE, Boomsma DI, et al. Quantitative genetic modeling of variation in human brain morphology. Cerebral cortex Sep 2001;11(9):816-824.

76. Gilmore JH, Schmitt JE, Knickmeyer RC, Smith JK, Lin W, Styner M, Gerig G, Neale MC. Genetic and environmental contributions to neonatal brain structure: A twin study. Human brain mapping Aug 2010;31(8):1174-1182.

77. Blokland GA, de Zubicaray GI, McMahon KL, Wright MJ. Genetic and environmental influences on neuroimaging phenotypes: a meta-analytical perspective on twin imaging studies. Twin research and human genetics : the official journal of the International Society for Twin Studies Jun 2012;15(3):351-371.

78. Voineskos AN. Genetic underpinnings of white matter 'connectivity': Heritability, risk, and heterogeneity in schizophrenia. Schizophrenia research Jan 2015;161(1):50-60.

79. Bohlken MM, Brouwer RM, Mandl RC, et al. Structural Brain Connectivity as a Genetic Marker for Schizophrenia. JAMA Psychiatry Jan 2016;73(1):11-19.

80. Wang F, Jiang T, Sun Z, et al. Neuregulin 1 genetic variation and anterior cingulum integrity in patients with schizophrenia and healthy controls. Journal of psychiatry \& neuroscience : JPN May 2009;34(3):181-186.

81. Hashimoto R, Numakawa T, Ohnishi T, et al. Impact of the DISC1 Ser704Cys polymorphism on risk for major depression, brain morphology and ERK signaling. Human molecular genetics Oct 15 2006;15(20):3024-3033.

82. Kennedy KM, Rodrigue KM, Land SJ, Raz N. BDNF Val66Met polymorphism influences age differences in microstructure of the Corpus Callosum. Front Hum Neurosci 2009;3:19.

83. Gurung R, Prata DP. What is the impact of genome-wide supported risk variants for schizophrenia and bipolar disorder on brain structure and function? A systematic review. Psychological medicine 2015;45(12):2461-2480.

84. Braskie MN, Kohannim O, Jahanshad N, et al. Relation between variants in the neurotrophin receptor gene, NTRK3, and white matter integrity in healthy young adults. Neuroimage Nov 15 2013;82:146-153.

85. DeLisi LE. The effect of cannabis on the brain: can it cause brain anomalies that lead to increased risk for schizophrenia? Curr Opin Psychiatry Mar 2008;21(2):140-150. 
86. Choi J, Jeong B, Polcari A, Rohan ML, Teicher MH. Reduced fractional anisotropy in the visual limbic pathway of young adults witnessing domestic violence in childhood. Neurolmage Jan 16 2012;59(2):1071-1079.

87. Jackowski AP, Douglas-Palumberi H, Jackowski M, Win L, Schultz RT, Staib LW, Krystal JH, Kaufman J. Corpus callosum in maltreated children with posttraumatic stress disorder: a diffusion tensor imaging study. Psychiatry research Apr 15 2008;162(3):256-261.

88. Cannon TD, van Erp TG, Rosso IM, et al. Fetal hypoxia and structural brain abnormalities in schizophrenic patients, their siblings, and controls. Archives of general psychiatry Jan 2002;59(1):35-41.

89. Haddad L, Schafer A, Streit F, et al. Brain structure correlates of urban upbringing, an environmental risk factor for schizophrenia. Schizophrenia bulletin Jan 2015;41(1):115-122.

90. Korver N, Quee PJ, Boos HB, Simons CJ, de Haan L, investigators G. Genetic Risk and Outcome of Psychosis (GROUP), a multi-site longitudinal cohort study focused on gene-environment interaction: objectives, sample characteristics, recruitment and assessment methods. International journal of methods in psychiatric research Sep 2012;21(3):205-221. 




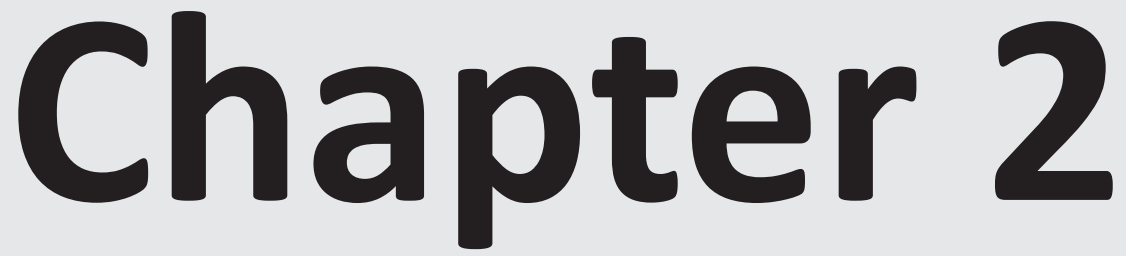

Microstructural white matter alterations in psychotic disorder: A family-based diffusion tensor imaging study

\author{
Patrick A.E. Domen ${ }^{1}$ \\ Stijn Michielse ${ }^{1^{*}}$ \\ Ed Gronenschild ${ }^{1^{*}}$ \\ Petra Habets ${ }^{1}$ \\ Alard Roebroeck ${ }^{3}$ \\ Koen Schruers ${ }^{1}$ \\ Jim van $\mathrm{Os}^{1,2}$ \\ Machteld Marcelis ${ }^{1}$
}

for G.R.O.U.P. 
${ }^{1}$ Department of Psychiatry and Neuropsychology, South Limburg Mental Health Research and Teaching Network, EURON, Maastricht University Medical Centre, Maastricht, The Netherlands.

${ }^{2}$ King's College London, King's Health Partners, Department of Psychosis Studies, Institute of Psychiatry, London, United Kingdom.

${ }^{3}$ Department of Cognitive Neuroscience, Maastricht University, Maastricht, The Netherlands

* Contributed equally

Published as:

Domen PA, Michielse S, Gronenschild E, et al. Microstructural white matter alterations in psychotic disorder: a family-based diffusion tensor imaging study. Schizophrenia Research. 2013; 146: 291-300. 


\section{ABSTRACT}

Background. There is evidence for microstructural white matter alterations in patients with psychotic disorder, suggesting altered interregional connectivity. Less is known about the presence and role of white matter alterations in well individuals at higher than average genetic risk for psychotic disorder.

Methods. 85 patients with psychotic disorder, 93 non-psychotic siblings of patients with psychotic disorder and 80 healthy controls underwent a diffusion tensor imaging (DTI) scanning protocol. In a whole brain voxel-based analysis using Tract Based Spatial Statistics (TBSS), fractional anisotropy (FA) values were compared between the three groups. Effects of antipsychotic medication and drug use were examined.

Results. The patients displayed significantly lower mean FA than the controls in the following regions: corpus callosum (genu, body, splenium), forceps major and minor, external capsule bilaterally, corona radiata (anterior, posterior) bilaterally, left superior corona radiata and posterior thalamic radiation bilaterally. Similar FA differences existed between the patients and the siblings; the siblings did not differ from the controls.

Conclusion. Profound microstructural white matter alterations were found in the corpus callosum and other tracti and fasciculi in the patients with psychotic disorder, but not in the siblings and the controls. These alterations may reflect brain pathology associated with the illness, illness-related environmental risk factors, or its treatment, rather than genetic risk. 


\section{Introduction}

There is growing evidence that cerebral vulnerability in schizophrenia may be mediated by altered connectivity between brain regions, rather than focal brain alterations. Indeed, neurophysiological and functional neuroimaging studies have demonstrated pathological functional connectivity ${ }^{1-3}$. Dysconnectivity, in terms of impaired axonal mechanisms and/or abnormal control of synaptic plasticity, may form the core pathology of schizophrenia ${ }^{4}$, and may be based in structural alterations. In support of this, volumetric MRI studies have shown decreased white matter volumes of the frontal lobes and temporo-parietal regions and a decreased corpus callosum volume (genu and/or truncus) in patients with schizophrenia ${ }^{5-7}$. Since the late nineties, numerous DTIstudies in patients with a diagnosis of schizophrenia have been published, showing a decrease in FA, indicative of white matter integrity loss, in several brain tracts, including the fronto-temporal connections, such as the arcuate fasciculus, anterior cingulum bundle, uncinate fasciculus ${ }^{8-10}$ and fronto-occipital tracts ${ }^{11,12}$. A meta-analysis in 2009 concluded that significant reductions were present in frontal deep white matter (genu corpus callosum, cingulum bundle, left anterior thalamic radiation, left corticobulbar tract and left inferior fronto-occipital fasciculus) and temporal deep white matter (splenium corpus callosum, fornix/stria terminalis, left inferior longitudinal fasciculus and left inferior fronto-occipital fasciculus) ${ }^{13}$. Although most, but not all studies ${ }^{14-16}$ report FA decreases associated with schizophrenia, there is inconsistency regarding the location of the affected brain regions, which, in part, may be related to differences in scanning protocol, study design (eg. characteristics of the participants) and analytical techniques. Structural dysconnectivity in patients with schizophrenia may reflect disease-related pathology, but may also represent expression of genetic risk for the disorder ${ }^{17}$. Thus, volumetric MRI studies in first-degree relatives ${ }^{18,19}$ and twins ${ }^{20}$ have shown indirect genetic effects on global grey and white matter volume reduction in schizophrenia. The number of DTI studies examining individuals at higher than average genetic risk for psychotic disorder is scant and sample sizes are, in general, small (number of high-risk individuals ranging from $n=16$ to $n=34$ ), except for one study ${ }^{21}$. The available evidence suggests that white matter alterations may be present in first-degree relatives without symptoms ${ }^{21-27}$. The results for so-called "ultra-high risk" samples with (pre)clinical symptoms are conflicting ${ }^{28}$, which likely is related to lack of consistency in ultra-high risk sample enrichment procedures across studies ${ }^{29}$ as well as to differences in brain regions studied and methodological approaches, precluding definite conclusions.

In the present large, DTI study $(n=258)$, whole-brain, voxel-based analytic techniques were used to examine patients with a psychotic disorder (highest genetic risk group), non-psychotic siblings (higher than average genetic risk group) of patients with a psychotic disorder, and healthy controls (average genetic risk group). We hypothesized 
that the patients with psychotic disorder would show reduced white matter integrity compared to the healthy controls, particularly in the corpus callosum, fronto-temporal, and fronto-parietal connections, with the siblings showing a pattern of alterations of intermediate severity.

\section{Methods}

\section{Participants}

The patients were recruited from an on-going longitudinal MRI study in Maastricht, the Netherlands. In selected representative geographical areas in the Netherlands and Belgium, the patients were identified through representative clinicians providing health care for patients with psychotic disorder. The siblings were contacted through the participating patients. Mailings and advertisements were effectuated in local newspapers of the same geographical area in order to recruit control participants. The total sample consisted of 258 participants: 85 patients with a psychotic disorder, 93 siblings without a psychotic disorder and 80 healthy controls. The sample included 56 families, of which 35 families contributed one patient and one healthy sibling, three families contributed one patient and two healthy siblings, and one family contributed one patient and three healthy siblings. One family contributed two patients, six families contributed two healthy siblings, and one family contributed three healthy siblings. In the control group, 9 families contributed two siblings. In addition, 44 families contributed a single patient, 34 families contributed a single sibling, and 62 families contributed a single control.

Inclusion criteria were: age range 16-50 years, a good command of Dutch language and for patients: a diagnosis of non-affective psychotic disorder with an illness duration of $<10$ years. The siblings and the controls did not have a lifetime diagnosis of any non-affective psychotic disorder. In addition, the controls had no first-degree relative with a lifetime diagnosis of any psychotic disorder, assessed using the Family Interview for Genetic Studies (FIGS) ${ }^{30}$.

Diagnosis was based on the Diagnostic and Statistical Manual of Mental Disorder-IV (DSM-IV) criteria ${ }^{31}$, measured with the Comprehensive Assessment of Symptoms and History (CASH) interview ${ }^{32}$. The patients were diagnosed as follows: schizophrenia $(n=59)$, schizoaffective disorder $(n=9)$, schizophreniform disorder $(n=4)$, brief psychotic disorder $(n=2)$, and psychotic disorder not otherwise specified $(n=11)$. Psychopathology in the siblings and controls was also assessed and respectively 18 and 12 participants had a history of a major depressive disorder. None of these met the criteria for a current depressive episode. 
All the participants were screened before MRI acquisition for the following exclusion criteria: brain injury with unconsciousness of greater than $1 \mathrm{~h}$, meningitis or other neurological diseases with possible impact on brain structure or function, cardiac arrhythmia requiring medical treatment and severe claustrophobia. In addition, subjects with metal corpora aliena were excluded from the study, as were women with intrauterine device status and (suspected) pregnancy.

The standing ethics committee approved the study protocol, and all the participants gave written informed consent in accordance with the committee's guidelines.

\section{Measures}

Level of psychotic symptomatology at the time of scanning was assessed with the Positive and Negative Symptom Scale (PANSS) ${ }^{33}$ in all three groups. The five factor model by van der Gaag and colleagues (2006), was used dividing the PANSS in positive symptoms, negative symptoms, disorganization symptoms, excitement and emotional distress ${ }^{34}$. The scores of the individual items of the 5 symptom dimensions were summed.

Educational level was defined as highest accomplished level of education. Handedness was assessed using the Annett Handedness Scale ${ }^{35}$.

In the patient group, antipsychotic (AP) medication use was determined by the patient's report and verified with the treating consultant psychiatrist. Best estimate lifetime (cumulative) AP use was determined by multiplying the number of days of AP use with the corresponding haloperidol equivalents and summing these scores for all periods of AP use (including the exposure period between baseline assessment for the G.R.O.U.P. study and the moment of baseline MRI scanning), using the recently published converting formulas for AP dose equivalents described by Andreasen and colleagues $^{36}$.

Substance use was measured with the Composite International Diagnostic Interview (CIDI) sections B-J-L ${ }^{37}$. Use of cannabis and other drugs was assessed as reported frequency of use during the last 12 months, as well as lifetime use. CIDI frequency data on lifetime cannabis and other drug use was available for respectively 250 participants (3\% missing data) and 256 participants (1\% missing data).

Alcohol use was defined as the reported number of weekly consumptions during the last 12 months.

\section{Image acquisition}

Magnetic resonance imaging scans were obtained at Maastricht University, the Netherlands, using an Allegra syngo MR A30 (Siemens, Erlangen, Germany) operating at $3.0 \mathrm{~T}$. The following anatomical scan parameters were used: Modified Driven Equilibrium Fourier Transform (MDEFT) sequence; 176 slices, $1 \mathrm{~mm}$ isotropic voxel 
size, echo time $2.4 \mathrm{~ms}$, repetition time $7.92 \mathrm{~ms}$, inversion time $910 \mathrm{~ms}$, flip angle $15^{\circ}$, total acquisition time $12 \mathrm{~min} 51 \mathrm{sec}$; Magnetization Prepared Rapid Acquisition Gradient-Echo (MPRAGE; Alzheimer's Disease Neuroimaging Initiative) sequence 192 slices, $1 \mathrm{~mm}$ isotropic voxel size, echo time $2.6 \mathrm{~ms}$, repetition time $2250 \mathrm{~ms}$, inversion time $900 \mathrm{~ms}$, flip angle $9^{\circ}$, total acquisition time $7 \mathrm{~min} 23 \mathrm{~s}$. The matrix size was 256 $\times 256$ and field of view was $256 \times 256 \mathrm{~mm}^{2}$. The number of excitations was one. Two sequences were used because of a scanner update during data collection.

Microstructural anatomy was examined using diffusion tensor imaging with an echoplanar-imaging sequence (field of view $230 \times 230 \mathrm{~mm}^{2}$, TR $10800 \mathrm{~ms}$, TE $84 \mathrm{~ms}$, voxel size $1.8 \times 1.8 \times 1.8 \mathrm{~mm}^{3}$, b-value $1000 \mathrm{~s} / \mathrm{mm}^{2}$, noise level 40,85 slices, no overlap). As a result of the scanner update, two DTI sequences were used: one with 76 directions (of which 4 T2-weighted (BO) and 72 diffusion-weighted (B)), and one with 81 directions ( $8 \times \mathrm{BO}$ and $73 \times \mathrm{B})$. The proportion of scans with 76 directions was balanced between the groups $(78 \%$ in the controls, $75 \%$ in the siblings and $69 \%$ in the patients $\left(\chi^{2}=1.52, P=0.468\right)$, preventing any systematic bias. Total acquisition time of the DTI sequence was $15 \mathrm{~min}$.

\section{Diffusion tensor imaging analysis}

Processing of DTI data was effectuated using tract-based spatial statistics (TBSS) v1.2 in FSL 4.1.6 (FMRIB Analysis Group, Oxford, UK, http://www.fmrib.ox.ac.uk/analysis/ research/tbss). First, standard Siemens DICOM files were transformed into compressed NIFTI format using a custom built in-house software named GIANT (General Image ANalysis Tools developed by EHBMG). Raw data were corrected for head movement and eddy currents invoked during scanning. The BO volume was skull-stripped using FSL's Brain Extraction Tool ${ }^{38}$ and this served as a brain mask for all B volumes.

The next step was fitting a diffusion tensor model at each voxel using data output from the brain extraction, diffusion weighted data and gradient directions following a general linear model (FreeSurfer v4.5.0, http://surfer.nmr.mgh.harvard.edu). After tensor fitting the process continued working on FA volumes, eroding them slightly. Nonlinear registration aligned each FA volume to $1 \times 1 \times 1 \mathrm{~mm}$ standard FMRIB58_FA space. The standard FMRIB58_FA contains a template derived from high-resolution images of 58 participants in a well-aligned population (both males and females ranging between 20 and 50 years of age) ${ }^{39}$.

After nonlinear transformation of the FA volumes into standard space, a mean FA skeleton from all the participants per group was derived. The mean FA skeleton follows the major white matter tracts in each individual participant (normalized in MNI152 space) and provides a way to compare between (groups of) the participants. The final step of the processing was setting the FA threshold using visual inspection of the FA skeleton, in the present study at a level of 0.25 , to include major white matter tracts while removing small peripheral tracts that would cause excess inter-participant vari- 
ability. In addition, this threshold setting avoided inclusion of regions that are likely to be composed of multiple tissue types or fiber orientations.

\section{Statistical analyses}

Voxel-wise statistical analysis was performed on the mean FA skeleton using a general linear model and applying FSL's randomize (v2.1) permutation-testing script ${ }^{40}$. The comparisons involved the statistical tests of group differences in FA, yielding a total of six contrasts: i) mean FA is lower (or higher) in the patients compared to the controls, ii) mean FA is lower (or higher) in the siblings compared to the controls, and iii) mean FA is lower (or higher) in the patients compared to the siblings. The a priori hypothesized confounding variables age, sex, handedness, level of education, lifetime cannabis use and lifetime other drug use were used in the statistical model. In addition to tests for regional group differences, stratified gender analyses were carried out.

In addition, associations between AP medication and FA were analyzed in two different ways. First, cumulative AP exposure was added as an additional covariate. Second, in the patients only, cumulative AP exposure was entered as independent (dummy) variable representing the distribution of scores divided by its tertiles (low, medium and high exposure) to examine the effect on FA within the patients.

Lastly, planned sensitivity analyses were performed excluding the siblings and controls with a history of affective disorder.

For the main analysis permutation tests were performed using 50,000 permutations in order to reduce the margin of error to acceptable uncertainty levels ( $P=0.05 \pm 0.00195$, ie. $3.9 \%$ of the nominal alpha). With regard to the additional analyses (effects of AP medication and gender, exclusion of affective disorder) 10,000 - 50,000 permutations were performed. The threshold free cluster enhancement (TFCE) option was enabled to find clusters without setting an initial cluster level ${ }^{41}$. Statistical maps (thresholded at $\mathrm{P}<0.05$ ) were used for assessing differences between groups (corrected for multiple comparisons).

The Johns Hopkins University International Consortium for Brain Mapping (JHU ICBM)-DTI-81 white-matter atlas labels ${ }^{42}$ were used to label significant voxels and assign a specific tract name. If the voxels did not match with the JHU ICBM labels, they were identified using the $\mathrm{JHU}$ white-matter tractography atlas ${ }^{43}$. A binary mask was created containing the significant clusters in order to extract FA values of the individual participants.

For visualization purposes (see Fig.1) and to extend the TBSS analyses, from all $38 \mathrm{JHU}$ labeled white matter tracts, skeleton mean FA values were extracted and exported to Stata version $12^{44}$. Regional group differences were tested with multilevel random regression procedures using the Stata XTREG command with adjustment for the a priori hypothesized confounding variables age, sex, handedness, and level of education. 
In addition, the data set was transformed from a wide to a long format, resulting in a hierarchically structured data set, with 38 regional FA measures (Level 1) nested in subjects (Level 2) who were part of the same families (Level 3). Because of the threelevel grouping structure of the data, compromising statistical independence of the observations, multilevel random regression models were fitted using the XTMIXED command in Stata with FA measures as the dependent variable and subject number and family number modelled as random effects. Mixed models contain both fixed and random effects, the fixed effects being analogous to standard regression coefficients (B).

Main effects of group and group $\times$ sex interactions in the model of FA were examined, as well as associations between cumulative AP dose and FA in the patient group. The same covariates were used as described above.

\section{Results}

\section{Descriptive analyses}

There were more women than men in the control group, whereas the opposite held for the patient group. The study comprised a relatively stable patient group as reflected by the low PANSS scores. The patients were more frequent cannabis- and other drug users than the siblings and controls (Table 1). At the time of scanning, seventy patients were receiving AP medication (second generation: $n=67$; first generation: $\mathrm{n}=3$ ). The mean current dosage of AP medication in terms of standard haloperidol equivalents was 5.5 milligrams $(\mathrm{mg})(\mathrm{SD}=4.6)$. Furthermore, 16 patients used antidepressants, 6 used benzodiazepines, 5 used anticonvulsants, and 2 used lithium. Three siblings and 3 control participants used antidepressants, and one control participant used benzodiazepines.

\section{Whole-brain group differences in FA}

Voxel-wise analysis in TBSS revealed significant differences in mean FA in two out of six comparisons, indicating that the patients had a lower mean FA than the controls and siblings. The number of voxels for each white matter tract with a significant higher FA $(P<0.05)$ in the controls and siblings, compared to patients, were derived and shown in Table 2. 

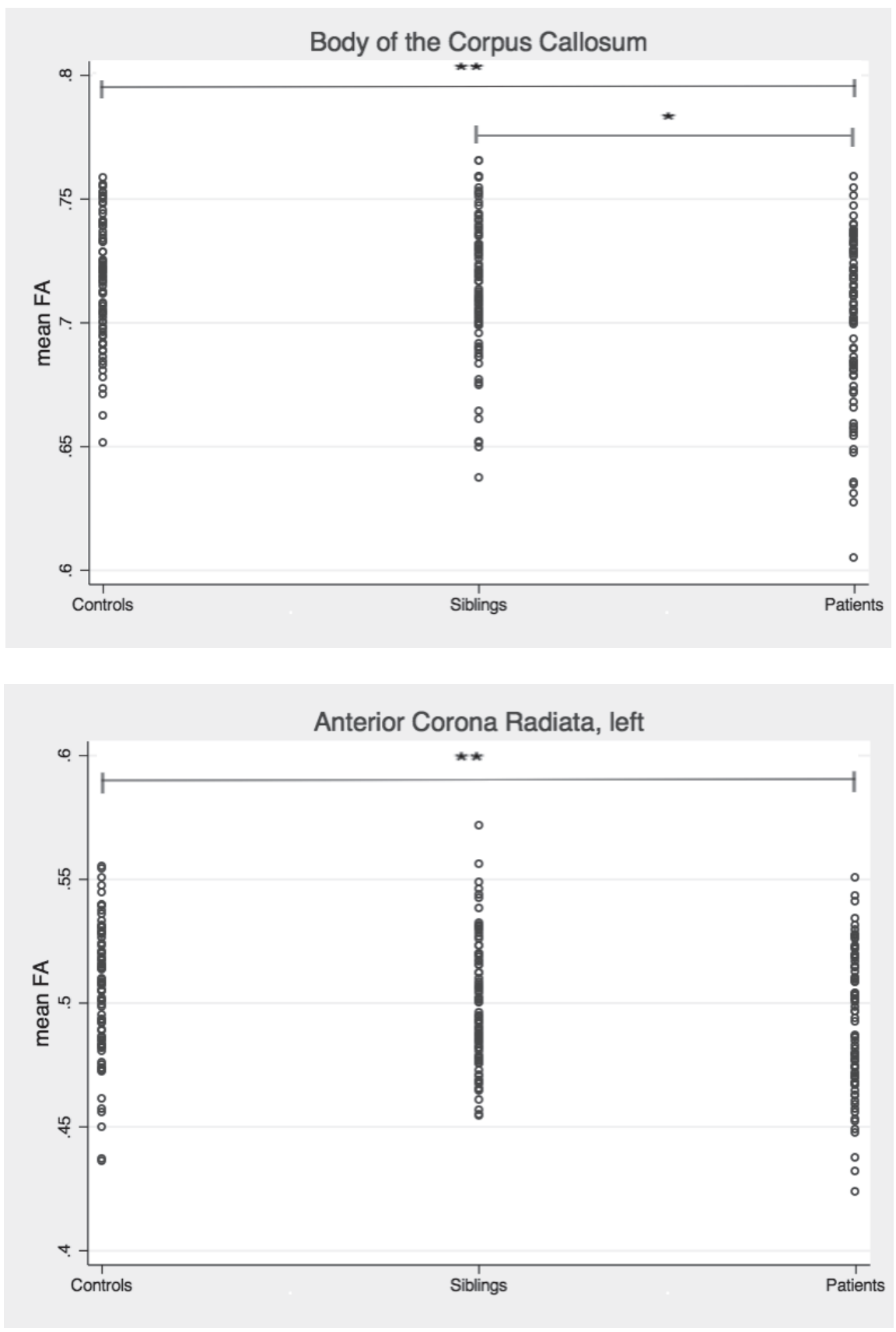

Fig. 1. Two white matter regions with a significantly lower mean FA value in the patients vs. the controls (**body of the corpus callosum: $\mathrm{B}=-.016, \mathrm{P}=0.001$, anterior corona radiata, left: $\mathrm{B}=$ $-.013, P=0.004$ ) and in the patients vs. the siblings (*body of the corpus callosum: $B=.013, P=$ $0.002)$; controlled for age, sex, handedness, highest level of education. 
A

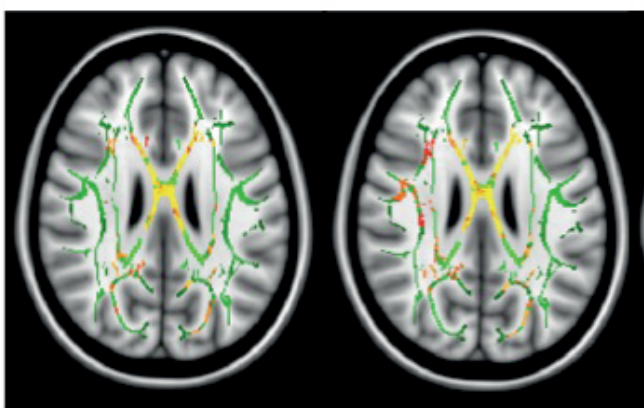

C

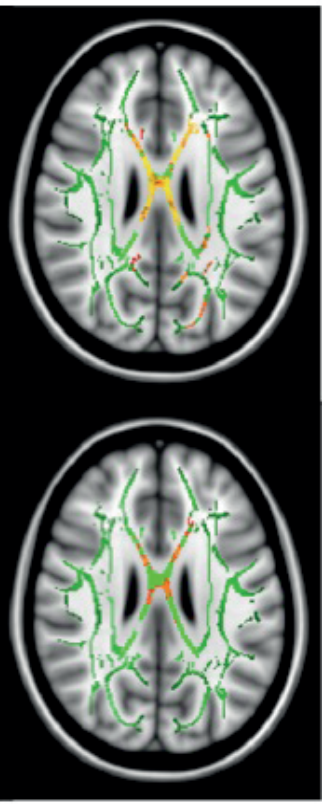

F

Fig. 2. Axial view of white matter regions, mainly the Corpus Callosum, in MNI space ( $x: 89$, $y: 107, z: 98)$, where the patients have significant lower mean FA values compared to the controls $(A, B, C)$ and the siblings $(D, E, F)$. All results were controlled for age, sex, handedness, highest level of education, and additionally for cannabis use (B, E) and cannabis and other drug use (C, F). Statistical maps were thresholded at $\mathrm{P}<0.05$, with the colormap reflecting the $\mathrm{P}$-values, ranging from 0.05 (red) to 0.00 (yellow). 
Table 1. Demographic characteristics of the participants

\begin{tabular}{|c|c|c|c|}
\hline & Controls $(n=80)$ & Siblings ( $n=93$ ) & Patients $(n=85)$ \\
\hline Sex Male (\%) & $29(36 \%)$ & $49(53 \%)$ & $58(68 \%)$ \\
\hline Handedness & 76.3 & 73.9 & 72.8 \\
\hline Age at scan (years) & $30.8 \pm 10.8$ & $29.4 \pm 8.8$ & $28.3 \pm 7.0$ \\
\hline Level of education & $5.4 \pm 1.8$ & $5.1 \pm 2.1$ & $4.1 \pm 2.0$ \\
\hline Age of onset (years) & & & $22.8 \pm 6.4$ \\
\hline Illness duration (years) & - & - & $5.4 \pm 3.6$ \\
\hline Antipsychotics ${ }^{a}$ & - & - & $6692.71 \pm 6254.18$ \\
\hline Cannabis $^{b}$ & $7.8 \pm 21.9$ & $19.3 \pm 37.2$ & $44.0 \pm 47.0$ \\
\hline Other drugs ${ }^{\mathrm{b}}$ & $0.90 \pm 4.7$ & $6.2 \pm 31.4$ & $42.4 \pm 90.8$ \\
\hline Alcohol $^{c}$ & $5.0 \pm 7.0$ & $9.8 \pm 17.3$ & $5.0 \pm 9.1$ \\
\hline \multicolumn{4}{|l|}{ PANSS } \\
\hline Positive symptoms & $7.3 \pm 1.1$ & $7.3 \pm 0.9$ & $10.4 \pm 5.0$ \\
\hline Negative symptoms & $8.2 \pm 1.0$ & $8.4 \pm 2.0$ & $12.0 \pm 5.9$ \\
\hline Disorganization & $10.2 \pm 1.2$ & $10.3 \pm 0.7$ & $12.5 \pm 4.1$ \\
\hline Excitement & $8.3 \pm 1.1$ & $8.6 \pm 1.4$ & $9.7 \pm 2.7$ \\
\hline Emotional Distress & $9.2 \pm 2.1$ & $9.9 \pm 2.6$ & $13.2 \pm 5.2$ \\
\hline
\end{tabular}

Means \pm SDs are reported

Abbreviations: PANSS, Positive and Negative Syndrome Scale

a Lifetime exposure in haloperidol equivalents

${ }^{\mathrm{b}}$ Mean number of times; life time

'Weekly consumptions of the last 12 months

Table 2. Group differences in FA: results from TBSS

\begin{tabular}{lccccc}
\hline \multicolumn{1}{c}{ Brain Area } & \multicolumn{2}{c}{$\begin{array}{c}\text { Siblings vs. } \\
\text { Patients }\end{array}$} & \multicolumn{2}{c}{$\begin{array}{c}\text { Controls vs. } \\
\text { Patients }\end{array}$} \\
\cline { 2 - 6 } & A & & B & C \\
\hline Genu of corpus callosum & 826 & 841 & 928 & 800 \\
Body of corpus callosum & 1983 & 2421 & 2581 & 2380 \\
Splenium of corpus callosum & 92 & 346 & 412 & 165 \\
Forceps major & 94 & 276 & 326 & 122 \\
Forceps minor & 1068 & 1038 & 1149 & 984 \\
Fornix (column and body of fornix) & 0 & 0 & 0 & 0 \\
Anterior limb of internal capsule, right & 0 & 49 & 398 & 0 \\
Anterior limb of internal capsule, left & 0 & 213 & 48 & 0 \\
Posterior limb of internal capsule, right & 0 & 0 & 22 & 0 \\
Posterior limb of internal capsule, left & 0 & 1 & 0 & 0 \\
\hline
\end{tabular}


Table 2. Group differences in FA: results from TBSS (continued)

\begin{tabular}{|c|c|c|c|c|}
\hline Brain Area & $\begin{array}{l}\text { Siblings vs. } \\
\text { Patients }\end{array}$ & & $\begin{array}{l}\text { ontrols v } \\
\text { Patients }\end{array}$ & \\
\hline & A & & B & $\mathrm{C}$ \\
\hline Retrolenticular part of internal capsule, right & 0 & 4 & 2 & 0 \\
\hline Retrolenticular part of internal capsule, left & 0 & 0 & 1 & 1 \\
\hline Anterior corona radiata, right & 0 & 1037 & 1049 & 750 \\
\hline Anterior corona radiata, left & 654 & 1157 & 1233 & 1120 \\
\hline Superior corona radiata, right & 395 & 241 & 394 & 175 \\
\hline Superior corona radiata, left & 221 & 468 & 470 & 382 \\
\hline Posterior corona radiata, right & 339 & 373 & 406 & 0 \\
\hline Posterior corona radiata, left & 13 & 442 & 443 & 416 \\
\hline Posterior thalamic radiation (incl. optic radiation), right & 253 & 244 & 255 & 0 \\
\hline Posterior thalamic radiation (incl. optic radiation), left & 0 & 313 & 352 & 260 \\
\hline $\begin{array}{l}\text { Sagittal stratum (incl. inf. longitudinal fasc. and inferior } \\
\text { fronto-occ. fasc), right }\end{array}$ & 149 & 3 & 8 & 0 \\
\hline $\begin{array}{l}\text { Sagittal stratum (incl. inf. longitudinal fasc. and inferior } \\
\text { fronto-occ. fasc), left }\end{array}$ & 0 & 5 & 46 & 35 \\
\hline External capsule, right & 0 & 255 & 394 & 0 \\
\hline External capsule, left & 0 & 371 & 425 & 400 \\
\hline Cingulum (cingulate gyrus), right & 0 & 57 & 140 & 127 \\
\hline Cingulum (cingulate gyrus), left & 23 & 0 & 0 & 0 \\
\hline Cingulum (hippocampus), right & 0 & 0 & 0 & 0 \\
\hline Cingulum (hippocampus), left & 0 & 0 & 0 & 0 \\
\hline Fornix, stria terminalis, right & 0 & 19 & 27 & 0 \\
\hline Fornix, stria terminalis, left & 0 & 4 & 18 & 16 \\
\hline Superior longitudinal fasciculus, right & 0 & 0 & 269 & 0 \\
\hline Superior longitudinal fasciculus, left & 0 & 184 & 198 & 173 \\
\hline Superior fronto-occipital fasciculus, right & 103 & 65 & 69 & 0 \\
\hline Superior fronto-occipital fasciculus, left & 0 & 64 & 62 & 0 \\
\hline Uncinate fasciculus, right & 0 & 50 & 57 & 0 \\
\hline Uncinate fasciculus, left & 0 & 53 & 58 & 56 \\
\hline Tapetum, right & 0 & 35 & 35 & 0 \\
\hline Tapetum, left & 0 & 5 & 5 & 5 \\
\hline
\end{tabular}

The number of voxels with a significant higher FA $(p<0.05)$ in the controls vs. the patients and the siblings vs. the patients. There were no regions with a significant higher FA in the siblings compared to the controls. Results were controlled (A) for age, sex, handedness, highest level of education, (B) age, sex, handedness, highest level of education and cannabis use, (C) age, sex, handedness, highest level of education, cannabis and other drug use. 
The patients showed a significantly lower FA compared to the control group in the following regions with $>250$ voxels and thresholded at $\mathrm{P}<0.05$ : corpus callosum (genu, body, splenium), forceps major and minor, external capsule bilaterally, corona radiata (anterior, posterior) bilaterally, left superior corona radiata and posterior thalamic radiation bilaterally. At a more conservative threshold $(P<0.01)$, the corpus callosum (genu, body), left external capsule en anterior corona radiata bilaterally remained significant (Fig. 2).

The mean FA values in the brain areas mentioned above showed a gradual decline from the controls to the siblings to patients (see Fig.1, results from multilevel random regression procedures in Stata). Although mean FA values in the siblings were generally lower than FA values in the controls, these differences were neither large nor conclusive statistically.

Compared to the siblings, the patients showed a significantly lower FA in the following regions with $>250$ voxels and thresholded at $\mathrm{P}<0.05$ : corpus callosum (genu, body), forceps minor, left anterior corona radiata, right superior and posterior corona radiata and the right posterior thalamic radiation. Only a few regions remained significant using a more conservative threshold, ie $\mathrm{P}<0.01$ : the right body of the corpus callosum and the right superior corona radiata (Fig. 2). All these regions overlapped with those standing out in the comparison between patients and healthy controls.

There were no regions with a higher mean FA in the patients compared to controls or with a higher mean FA in the siblings compared to the controls.

\section{Additional covariates: cannabis and other drug use}

The results described above were controlled for age, sex, handedness, and highest level of education. The analyses were repeated using the additional covariates of cannabis and other drug use. When only cannabis use was added as an additional covariate, the number of significant brain regions showing FA difference between the patients and the controls, as well the number of voxels per region was comparable to the analysis without cannabis use as a covariate. The right superior longitudinal fasciculus became apparent as an additional area with a significant higher FA in controls than in the patients (see Table 2).

When both cannabis and other drugs were added as covariates, the number of white matter areas with significant FA differences between the patients and the controls slightly decreased, particularly on the right side of the brain. Effect sizes of the genu and body of the corpus callosum held, whereas the effect size of the splenium was decreased (Table 2). Overall, the effect of drug use on white matter tissue appeared to be minor in this sample. 


\section{AP medication}

When cumulative AP exposure was added in TBSS as an additional covariate, the number of significant voxels decreased markedly, but significantly lower FA in the patients compared to the controls remained present in the body of the corpus callosum and the right posterior corona radiata (> 250 voxels). The patient-sibling comparison did not show significant regional differences.

Cumulative AP exposure was also entered as an independent (dummy) variable (low exposure: $n=22$, medium exposure: $n=22$ and high exposure: $n=21$ ) to examine the effect on FA within the patients. No significant differences in mean FA values were found when high or medium AP exposure levels were compared to low AP exposure levels.

In addition, analysis in Stata showed that there was no significant association between lifetime AP use and $F A\left(B=1.4 \times 10^{-7}, P=0.68\right)$.

\section{Sex differences}

Stratified TBSS analyses were done separating the male ( $n=29$ controls, 49 siblings, 58 patients) and the female subjects ( $n=51$ controls, 44 siblings, 27 patients). This led to $50 \%$ reduction in sample size and to an absence of significant regional differences in mean FA in the patient-control, patient- sibling and control-sibling comparisons.

Since the number of the participants varied considerably between the groups after separating them by sex (eg. far more male patients than controls, and vice versa for females), the analyses were repeated in equally sized groups, ie. three groups of 29 males and three groups of 27 females. The total number of significant voxels markedly decreased: $8 \%$ (=1291 voxels) left in the female patient-control comparison, and $28 \%$ (=1845 voxels) left in the male patient-sibling comparison. In females, the patient group showed significantly lower FA values compared to the controls, but not compared to the siblings, in the genu and body of the corpus callosum and the forceps minor (> 250 voxels). In males, the patient group showed significantly lower mean FA values compared to the siblings, but not compared to the controls, following a similar regional pattern as in the whole group analysis, with only the body of the corpus callosum exceeding 250 voxels. There were no significant mean FA differences in the sibling-control comparisons.

In addition, although there was a significant main effect of group in the model of FA (group, linear trend: $B=-0.004, P=0.02$; patients compared to controls: $B=-0.007$, $\mathrm{P}=0.02$; siblings compared to controls: $\mathrm{B}=-0.002, \mathrm{P}=0.45$ ), the group $\times$ sex interaction was not significant $\left(\mathrm{chi}^{2}: 1.04, \mathrm{P}=0.60\right)$, suggesting that the effect of group is not conditional on sex. 


\section{Exclusion of individuals with a history of affective disorder}

Repeating the original analyses excluding siblings ( 8 males and 10 females) and controls ( 1 male and 11 females) with a history of affective disorder resulted in an overall increase $(+15 \%)$ of significant voxels in the patient-control comparison, and an overall decrease $(-76 \%)$ of significant voxels in the patient-sibling comparison. The regional pattern of patient-related decreased FA was not changed, except for the absence of the left anterior, superior and posterior corona radiata in the patient-sibling comparison. No significant differences in mean FA values were found between the controls and the siblings.

\section{Discussion}

In this whole-brain DTI study, patients with a psychotic disorder, non-psychotic siblings and healthy controls were examined in order to identify microstructural white matter alterations that may be associated with the familial risk for schizophrenia. The results showed a gradual decline of mean FA from the controls to the siblings to the patients. Mean FA values were significantly lower in the patients compared to the controls in several major white matter tracts, while differences between the siblings and the controls did not reach statistical significance. Thus, microstructural white matter alterations may reflect brain pathology associated with the illness, illness related environmental factors, or its treatment, rather than genetic risk.

\section{Findings in the patients}

The results support the hypothesis that the patients with psychotic disorder compared to the healthy controls have reduced connectivity in several brain regions, while no regions with increased FA were identified. This is in line with the majority

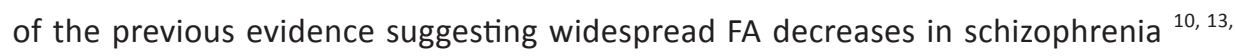
${ }^{28}$. A significantly lower mean FA in the patients in certain brain fasciculi and tracts may indicate dysfunctional axonal connectivity, which has been related to psychotic symptomatology. For example, smaller FA in the left superior longitudinal fasciculus has been associated with positive symptoms ${ }^{45}$, in particular hallucinations ${ }^{46}$ and smaller FA in posterior callosal fibers has been associated with negative symptoms ${ }^{46}$. In the present study, the most prominent FA decreases were found in the corpus callosum, in particular the anterior part (genu) of the corpus callosum. The corpus callosum and its postulated role in the dysconnectivity hypothesis in schizophrenia are highlighted in several studies, but there is no consensus about which part of the corpus callosum is most affected; the anterior part ${ }^{47}$ or the more posterior interhemispheric connections ${ }^{48}$. The results from the present study showed that 
the anterior-middle part was most affected, but also suggest that the entire corpus callosum may in fact be involved. These FA decreases in the corpus callosum provide additional evidence for aberrant cerebral interhemispheric activity in callosal fibers, further strengthening the theory that dysconnection in white matter connectional architecture in this part of the brain plays an important role in the core pathology of schizophrenia ${ }^{49,50}$. Indeed, aberrant connectivity in the corpus callosum has been associated with social cognition and interhemispheric information transfer deficits ${ }^{51}$, 52 , as well as positive and negative symptoms ${ }^{14,53}$.

\section{Findings in the siblings}

Only a few studies with relatively small samples sizes, have examined FA in first-degree relatives of patients with psychotic disorder. Clark et al. (2011) found decreased FA in both patients and non-psychotic first-degree relatives in the left and right inferior longitudinal fasciculus, the left inferior fronto-occipital fasciculus and the temporal component of the superior longitudinal fasciculus, using a ROI approach ${ }^{23}$. The effect sizes for the relatives were intermediate to those for the patients and controls. Other studies have reported significant white matter abnormalities in first-degree relatives compared to controls in the prefrontal cortex and hippocampus ${ }^{24}$, medial frontal regions ${ }^{22}$, the anterior limb of the internal capsule ${ }^{26}$ and the corpus callosum ${ }^{25}$. One recent family study, comprising larger samples than hitherto, reported absence of FA alterations in most white matter tracts in siblings as compared to patients with schizophrenia and controls, except for an increased FA in the right arcuate fasciculus ${ }^{21}$. Although in the present study, the mean FA in the significant areas between the controls and the patients reflected a dose response effect of familial risk in that the values were highest in controls and lowest in the patients, with intermediate values for the siblings, there were no significant differences between the sibling and the control group. In addition, there were significant changes in the patient versus sibling comparison, suggesting that the siblings are more similar to the controls than the patients. In other words, the present study does not provide strong evidence for a microstructural white matter intermediate phenotype, but rather suggests that the microstructural white matter alterations are contingent on the expression of psychotic illness. As cross-sectional studies do not allow conclusions on direction of effect, fiber dysconnectivity could in fact arise from the disease process or disease-related differential exposure to environmental risk factors or medication use.

\section{Antipsychotic medication}

Qualitative reviews have suggested that AP use may affect brain structure ${ }^{54,55}$. A recent, fairly large longitudinal study found that white matter volume loss was associated with more extensive use of antipsychotics even after adjustment for illness 
duration, illness severity, and substance abuse ${ }^{56}$. However, cross-sectional DTI studies with patients who were either medication-naïve or briefly medicated did not find evidence for a significant association between FA and antipsychotic exposure ${ }^{57,58}$. In the present study, extensive TBSS and Stata analyses both supported absence of significant effects of cumulative AP exposure on FA, except for one specific analysis in TBSS, in which AP exposure was added as a covariate and, consequently, the number of significant voxels between the patients and the controls markedly decreased (by 79\%). This, however, likely is an effect of the randomize procedure in FSL, where all controls and siblings receive a zero value for AP exposure leading to skewed distributions in the equation and distortion of the results (i.e. controlling for AP is like controlling for patient-status itself). Moreover, despite the voxel reduction in this analysis, significantly lower FA in the patients compared to the controls remained present in the body of the corpus callosum and the right posterior corona radiate (> 250 voxels), showing the robustness of FA differences in these areas. Lastly, altered FA values have been detected in AP-naïve patients ${ }^{59-61}$, suggesting that AP exposure may at best contribute to, but not fully explain, the white matter alterations.

\section{Environmental factors}

Specific (epi)genetic or environmental effects and/or gene-environment interplay may have contributed to the emergence of structural dysconnectivity in patients. Evidence for gene effects has come from studies with healthy subjects and studies with patients showing associations between polymorphisms of myelin-associated genes, such as NRG1, ErbB4 and PIK4CA on the one hand, and white matter integrity in the anterior cingulum, the anterior limb of the internal capsule and frontotemporal fibers on the other $^{17,62-65}$.

Certain environmental factors, such as childhood trauma ${ }^{66}$ and urban upbringing ${ }^{67}$ have been associated with cerebral alterations in respectively traumatized and non-psychiatric populations. Although these environments are risk factors for schizophrenia ${ }^{68}$ they have never been examined in relation to cerebral white matter in this patient population. The influence of cannabis on white matter volume in schizophrenia is weak ${ }^{69,70}$ but DTI studies have shown both FA increases and decreases in cannabis-using patients with schizophrenia compared to non-using patients ${ }^{28,}$ ${ }^{71}$. Previous analyses on the present study sample have demonstrated that familial predisposition for psychotic disorder is associated with greater cannabis-induced reduction in cortical thickness ${ }^{72}$. The current DTI results suggest that there is no marked effect of cannabis on microstructural white matter, as the controlling TBSS analyses for cannabis use did not change the results. However, the number of white matter areas that were significantly different between the patients and the controls slightly decreased in the model that included other drug use as a covariate. This may indi- 
cate that non-cannabis drug use may have a subtle, though measurable, effect on microstructural white matter in patients with psychotic disorder. To date, there are no other DTI studies reporting on an association between FA and non-cannabis drug use in individuals with psychotic disorder. In healthy (non-psychotic) individuals, other drug use (cocaine, heroin) has been negatively associated with FA in several brain areas, including the corpus callosum ${ }^{73-75}$. Thus, other drugs may affect the integrity of white matter brain tracts in individuals with psychotic disorder as well as in healthy (non-psychotic) individuals.

The possible influence of environmental risk factors on white matter tissue warrants further investigation.

\section{Sex differences}

Several studies have addressed sex differences in FA in healthy individuals. For the corpus callosum, both higher FA values in women compared to men ${ }^{76}$, higher FA values in men compared to women ${ }^{77}$, as well as no sex differences ${ }^{78}$ have been reported. In patients with schizophrenia, the literature on sex differences in FA is limited, but suggests lower values in women compared to men in the genu ${ }^{79}$ and the whole corpus callosum ${ }^{80}$. In the present study, the absence of significant group differences in regional FA in males and females separately could be the result of lack of power and markedly unequal group sizes after stratification by sex. Indeed, when stratified analyses were done in equally sized groups, there were patient-control differences in females, but not in males, and sibling-patient differences in males, but not in females. Multilevel analyses in Stata, however, did not yield support for a group $\times$ sex interaction. The contrasting results between TBSS and Stata analyses could be the result of methodological differences (voxelwise analyses of aligned FA data projected on the mean FA skeleton, versus multilevel regression analyses on the skeleton mean FA's of 38 regions).

In conclusion, the significant FA reductions in the patients may be more female than male driven, which needs replication in larger samples. The absence of reduced FA in the siblings compared to the controls, however, was consistent in all analyses, thus consistently providing evidence for the absence of an intermediated phenotype.

\section{Methodological considerations}

Since FA decreases in cortical and subcortical areas have been found in patients with major depression ${ }^{81}$, a priori planned sensitivity analyses were done excluding all individuals with a history of affective disorder. Although the number of significant voxels varied, which may be due to a different sex distribution between the groups as the majority of the excluded participants were females, it did not alter the regional pattern of significant white matter tracts. 
Structural neuroimaging studies often differ in scanning acquisition and imaging processing. These differences could have contributed to discrepancies in outcome. The present study was the second family study using a 3T scanner. The signal-to-noise ratio using this field strength is better, as is the visibility of certain details compared to a 1.5T scanner. Also, the sequence alteration due to a scanner update could in theory have influenced our results. However, we repeated the TBSS analyses, with the number of scan directions as additional covariate. The number and pattern of significant findings was not affected (results available on request).

Other methodological differences are reflected in the type of analyses: hypothesis-generating whole-brain voxel-wise analysis versus the hypothesis-testing ROI approach. It is known that due to image misregistration, smoothing and partial volume effects, whole brain voxel-wise analysis often yields different results compared to the region of interest (ROI) studies ${ }^{82,83}$.

In DTI studies, different atlases are used in establishing the exact regions of white matter alterations. These atlases differ considerably in the definitions of the boundaries of particular white matter tracts and of the labeling of certain brain regions, in an attempt to account for overlap and crossing fibers. We used two versions of the white matter JHU atlas ${ }^{42,43}$ to include most major white matter tracts. The differences in atlases among the DTI studies hamper between-study comparisons.

\section{Conclusion}

This large cross-sectional DTI study examined white matter integrity in relation to different levels of familial risk for psychotic disorder. There was evidence for patientspecific alterations in microstructural white matter, most profoundly present in the corpus callosum, with no evidence for an intermediate phenotype. The widespread disruptions in white matter in patients with schizophrenia may reflect disease-related dysconnectivity, or disease-related differential exposure to environmental risk factors contributing to the symptoms of schizophrenia. 


\section{References}

1. Andreasen NC, Paradiso S, O' Leary DS. "Cognitive dysmetria" as an integrative theory of schizophrenia; a dysfunction in cortical - subcortical - cerebellar circuitry. Schizophrenia Bulletin 1998;24(2):203-218.

2. Friston KJ, Frith CD. Schizophrenia: a disconnection syndrome? Clin Neurosci 1995;3(2):89-97.

3. Konrad A, Winterer G. Disturbed structural connectivity in schizophrenia primary factor in pathology or epiphenomenon? Schizophrenia Bulletin 2008;34(1):72-92.

4. Friston KJ. The Disconnection hypothesis. Schizophrenia research 1998;30:115-125.

5. Walterfang M, Wood SJ, Velakoulis D, Pantelis C. Neuropathological, neurogenetic and neuroimaging evidence for white matter pathology in schizophrenia. Neurosci Biobehav Rev 2006;30(7):918-948.

6. Olabi B, Ellison-Wright I, McIntosh AM, Wood SJ, Bullmore E, Lawrie SM. Are there progressive brain changes in schizophrenia? A meta-analysis of structural magnetic resonance imaging studies. Biol Psychiatry Jul 1 2011;70(1):88-96.

7. Makris N, Seidman LJ, Ahern T, Kennedy DN, Caviness VS, Tsuang MT, Goldstein JM. White matter volume abnormalities and associations with symptomatology in schizophrenia. Psychiatry Res Jul 30 2010;183(1):21-29.

8. Burns J, Job D, Bastin ME, Whalley $H$, Macgillivray $T$, Johnstone EC, Lawrie SM. Structural disconnectivity in schizophrenia: a diffusion tensor magnetic resonance imaging study. $\mathrm{Br} J$ Psychiatry May 2003;182:439-443.

9. Kanaan RA, Kim JS, Kaufmann WE, Pearlson GD, Barker GJ, McGuire PK. Diffusion tensor imaging in schizophrenia. Biol Psychiatry Dec 15 2005;58(12):921-929.

10. Kubicki M, McCarley R, Westin CF, Park HJ, Maier S, Kikinis R, Jolesz FA, Shenton ME. A review of diffusion tensor imaging studies in schizophrenia. J Psychiatr Res Jan-Feb 2007;41(1-2):1530.

11. Ardekani BA, Nierenberg J, Hoptman MJ, Javitt DC, Lim KO. MRI study of white matter diffusion anisotropy in schizophrenia. Neuroreport Nov 14 2003;14(16):2025-2029.

12. Mitelman SA, Torosjan $\mathrm{Y}$, Newmark RE, et al. Internal capsule, corpus callosum and long associative fibers in good and poor outcome schizophrenia: a diffusion tensor imaging survey. Schizophr Res May 2007;92(1-3):211-224.

13. Ellison-Wright I, Bullmore E. Meta-analysis of diffusion tensor imaging studies in schizophrenia. Schizophrenia research Mar 2009;108(1-3):3-10.

14. Hubl D, Koenig T, Strik W, et al. Pathways that make voices: white matter changes in auditory hallucinations. Arch Gen Psychiatry Jul 2004;61(7):658-668.

15. Price G, Bagary MS, Cercignani M, Altmann DR, Ron MA. The corpus callosum in first episode schizophrenia: a diffusion tensor imaging study. J Neurol Neurosurg Psychiatry Apr 2005;76(4):585-587.

16. Steel RM, Bastin ME, McConnell S, Marshall I, Cunningham-Owens DG, Lawrie SM, Johnstone EC, Best JJ. Diffusion tensor imaging (DTI) and proton magnetic resonance spectroscopy $(1 \mathrm{H}$ MRS) in schizophrenic subjects and normal controls. Psychiatry Res May 30 2001;106(3):161170.

17. Marenco S, Radulescu E. Imaging genetics of structural brain connectivity and neural integrity markers. Neurolmage Nov 15 2010;53(3):848-856. 
18. Goghari VM, Rehm K, Carter CS, MacDonald AW, 3rd. Regionally specific cortical thinning and gray matter abnormalities in the healthy relatives of schizophrenia patients. Cereb Cortex Feb 2007;17(2):415-424.

19. McDonald C, Bullmore ET, Sham PC, Chitnis X, Wickham H, Bramon E, Murray RM. Association of genetic risks for schizophrenia and bipolar disorder with specific and generic brain structural endophenotypes. Arch Gen Psychiatry Oct 2004;61(10):974-984.

20. Hulshoff Pol HE, Schnack HG, Mandl RC, et al. Gray and white matter density changes in monozygotic and same-sex dizygotic twins discordant for schizophrenia using voxel-based morphometry. Neurolmage Jun 2006;31(2):482-488.

21. Boos HB, Mandl RC, van Haren NE, Cahn W, van Baal GC, Kahn RS, Hulshoff Pol HE. Tract-based diffusion tensor imaging in patients with schizophrenia and their non-psychotic siblings. Eur Neuropsychopharmacol 2013 Apr;23(4):295-304.

22. Camchong J, Lim KO, Sponheim SR, Macdonald AW. Frontal white matter integrity as an endophenotype for schizophrenia: diffusion tensor imaging in monozygotic twins and patients' nonpsychotic relatives. Front Hum Neurosci 2009;3:35.

23. Clark KA, Nuechterlein KH, Asarnow RF, et al. Mean diffusivity and fractional anisotropy as indicators of disease and genetic liability to schizophrenia. J Psychiatr Res Jul 2011;45(7):980988.

24. Hao Y, Yan Q, Liu H, et al. Schizophrenia patients and their healthy siblings share disruption of white matter integrity in the left prefrontal cortex and the hippocampus but not the anterior cingulate cortex. Schizophrenia research Oct 2009;114(1-3):128-135.

25. Knochel C, Oertel-Knochel V, Schonmeyer R, et al. Interhemispheric hypoconnectivity in schizophrenia: fiber integrity and volume differences of the corpus callosum in patients and unaffected relatives. Neurolmage Jan 16 2012;59(2):926-934.

26. Munoz Maniega S, Lymer GK, Bastin ME, et al. A diffusion tensor MRI study of white matter integrity in subjects at high genetic risk of schizophrenia. Schizophrenia research Dec 2008;106(2-3):132-139.

27. Narr KL, Hageman N, Woods RP, et al. Mean diffusivity: a biomarker for CSF-related disease and genetic liability effects in schizophrenia. Psychiatry Res Jan 30 2009;171(1):20-32.

28. Peters BD, Blaas J, de Haan L. Diffusion tensor imaging in the early phase of schizophrenia: what have we learned? J Psychiatr Res Nov 2010;44(15):993-1004.

29. van Os J, Linscott RJ. Introduction: the extended psychosis phenotype--relationship with schizophrenia and with ultrahigh risk status for psychosis. Schizophrenia Bulletin Mar 2012;38(2):227-230.

30. Maxwell M. Family Interview for Genetic Studies (FIGS): Manual For FIGS. Clinical Neurogenetics Branch, Intramural Research Program, National Institute of Mental Health, Bethesda, MD 1992.

31. APA. Diagnostic and statistical manual of mental disorders, 4th ed. Washington, DC; American Psychiatric Association 2000.

32. Andreasen NC, Flaum M, Arndt S. The Comprehensive Assessment of Symptoms and History (CASH). An instrument for assessing diagnosis and psychopathology. Arch Gen Psychiatry Aug 1992;49(8):615-623.

33. Kay SR, Fiszbein A, Opler LA. The positive and negative syndrome scale (PANSS) for schizophrenia. Schizophr Bull 1987;13(2):261-276. 
34. van der Gaag $M$, Hoffman $T$, Remijsen $M$, et al. The five-factor model of the Positive and Negative Syndrome Scale II: a ten-fold cross-validation of a revised model. Schizophrenia Research Jul 2006;85(1-3):280-287.

35. Annett M. A classification of hand preference by association analysis. British Journal of Psychology 1970;61(3):303-321.

36. Andreasen NC, Pressler M, Nopoulos P, Miller D, Ho BC. Antipsychotic dose equivalents and dose-years: a standardized method for comparing exposure to different drugs. Biological Psychiatry Feb 1 2010;67(3):255-262.

37. WHO. Composite International Diagnostic Interview (CIDI). 1990(Geneva: World Health Organization).

38. Smith SM. Fast robust automated brain extraction. Human brain mapping Nov 2002;17(3):143155.

39. Smith SM, Jenkinson $M$, Johansen-Berg $H$, et al. Tract-based spatial statistics: voxelwise anaIysis of multi-subject diffusion data. Neurolmage Jul 15 2006;31(4):1487-1505.

40. Smith SM, Jenkinson M, Woolrich MW, et al. Advances in functional and structural MR image analysis and implementation as FSL. Neurolmage 2004;23 Suppl 1:S208-219.

41. Smith SM, Nichols TE. Threshold-free cluster enhancement: addressing problems of smoothing, threshold dependence and localisation in cluster inference. Neurolmage Jan 1 2009;44(1):83-98.

42. Mori $\mathrm{S}$, Oishi $\mathrm{K}$, Jiang $\mathrm{H}$, et al. Stereotaxic white matter atlas based on diffusion tensor imaging in an ICBM template. Neurolmage Apr 1 2008;40(2):570-582.

43. Hua K, Zhang J, Wakana S, et al. Tract probability maps in stereotaxic spaces: analyses of white matter anatomy and tract-specific quantification. Neurolmage Jan 1 2008;39(1):336-347.

44. StataCorp. Stata Statistical Software: Release 12. College Station, TX: StataCorp LP 2009.

45. Skelly LR, Calhoun V, Meda SA, Kim J, Mathalon DH, Pearlson GD. Diffusion tensor imaging in schizophrenia: relationship to symptoms. Schizophr Res Jan 2008;98(1-3):157-162.

46. Seok JH, Park HJ, Chun JW, Lee SK, Cho HS, Kwon JS, Kim JJ. White matter abnormalities associated with auditory hallucinations in schizophrenia: a combined study of voxel-based analyses of diffusion tensor imaging and structural magnetic resonance imaging. Psychiatry Research Nov 15 2007;156(2):93-104.

47. Kubicki M, Styner M, Bouix S, et al. Reduced interhemispheric connectivity in schizophreniatractography based segmentation of the corpus callosum. Schizophr Res Dec 2008;106(23):125-131.

48. Patel S, Mahon K, Wellington R, Zhang J, Chaplin W, Szeszko PR. A meta-analysis of diffusion tensor imaging studies of the corpus callosum in schizophrenia. Schizophr Res Jul 2011;129(23):149-155.

49. Zalesky A, Fornito A, Seal ML, Cocchi L, Westin CF, Bullmore ET, Egan GF, Pantelis C. Disrupted axonal fiber connectivity in schizophrenia. Biological Psychiatry Jan 1 2011;69(1):80-89.

50. Woodruff PW, Phillips ML, Rushe T, Wright IC, Murray RM, David AS. Corpus callosum size and inter-hemispheric function in schizophrenia. Schizophrenia Research Feb 28 1997;23(3):189196.

51. Chaim TM, Schaufelberger MS, Ferreira LK, et al. Volume reduction of the corpus callosum and its relationship with deficits in interhemispheric transfer of information in recent-onset psychosis. Psychiatry Research Oct 30 2010;184(1):1-9.

52. Miyata J, Yamada M, Namiki C, et al. Reduced white matter integrity as a neural correlate of social cognition deficits in schizophrenia. Schizophrenia Research Jun 2010;119(1-3):232-239. 
53. Rotarska-Jagiela A, Schonmeyer R, Oertel V, Haenschel C, Vogeley K, Linden DE. The corpus callosum in schizophrenia-volume and connectivity changes affect specific regions. Neuroimage 2008;39(4):1522-1532.

54. Navari S, Dazzan P. Do antipsychotic drugs affect brain structure? A systematic and critical review of MRI findings. Psychol Med Nov 2009;39(11):1763-1777.

55. Smieskova R, Fusar-Poli $P$, Allen $P$, et al. The effects of antipsychotics on the brain: what have we learnt from structural imaging of schizophrenia?--a systematic review. Curr Pharm Des 2009;15(22):2535-2549.

56. Ho BC, Andreasen NC, Ziebell S, Pierson R, Magnotta V. Long-term antipsychotic treatment and brain volumes: a longitudinal study of first-episode schizophrenia. Archives of General Psychiatry Feb 2011;68(2):128-137.

57. Peters BD, de Haan L, Dekker N, et al. White matter fibertracking in first-episode schizophrenia, schizoaffective patients and subjects at ultra-high risk of psychosis. Neuropsychobiology 2008;58(1):19-28.

58. Szeszko PR, Robinson DG, Ashtari M, et al. Clinical and neuropsychological correlates of white matter abnormalities in recent onset schizophrenia. Neuropsychopharmacology : official publication of the American College of Neuropsychopharmacology Apr 2008;33(5):976-984.

59. Cheung V, Cheung C, McAlonan GM, et al. A diffusion tensor imaging study of structural dysconnectivity in never-medicated, first-episode schizophrenia. Psychol Med Jun 2008;38(6):877-885.

60. Gasparotti R, Valsecchi P, Carletti F, Galluzzo A, Liserre R, Cesana B, Sacchetti E. Reduced fractional anisotropy of corpus callosum in first-contact, antipsychotic drug-naive patients with schizophrenia. Schizophr Res Mar 2009;108(1-3):41-48.

61. Zou LQ, Xie JX, Yuan HS, Pei XL, Dong WT, Liu PC. Diffusion tensor imaging study of the anterior limb of internal capsules in neuroleptic-naive schizophrenia. Acad Radiol Mar 2008;15(3):285289.

62. Mclntosh AM, Moorhead TW, Job D, et al. The effects of a neuregulin 1 variant on white matter density and integrity. Molecular psychiatry Nov 2008;13(11):1054-1059.

63. Zuliani R, Moorhead TW, Bastin ME, et al. Genetic variants in the ErbB4 gene are associated with white matter integrity. Psychiatry research Feb 28 2011;191(2):133-137.

64. Konrad A, Vucurevic G, Musso F, Stoeter P, Dahmen N, Winterer G. ErbB4 genotype predicts left frontotemporal structural connectivity in human brain. Neuropsychopharmacology Feb 2009;34(3):641-650.

65. Wang F, Jiang T, Sun Z, et al. Neuregulin 1 genetic variation and anterior cingulum integrity in patients with schizophrenia and healthy controls. Journal of psychiatry \& neuroscience : JPN May 2009;34(3):181-186.

66. Andersen SL, Tomada A, Vincow ES, Valente E, Polcari A, Teicher MH. Preliminary evidence for sensitive periods in the effect of childhood sexual abuse on regional brain development. $J$ Neuropsychiatry Clin Neurosci Summer 2008;20(3):292-301.

67. Lederbogen F, Kirsch P, Haddad L, et al. City living and urban upbringing affect neural social stress processing in humans. Nature Jun 23 2011;474(7352):498-501.

68. van Os J, Kenis G, Rutten BP. The environment and schizophrenia. Nature Nov 11 2010;468(7321):203-212.

69. Cahn W, Hulshoff Pol HE, Caspers E, van Haren NE, Schnack HG, Kahn RS. Cannabis and brain morphology in recent-onset schizophrenia. Schizophrenia research Apr 1 2004;67(2-3):305307. 
70. Solowij N, Yucel M, Respondek C, Whittle S, Lindsay E, Pantelis C, Lubman DI. Cerebellar white-matter changes in cannabis users with and without schizophrenia. Psychological medicine Nov 2011;41(11):2349-2359.

71. DeLisi LE. The effect of cannabis on the brain: can it cause brain anomalies that lead to increased risk for schizophrenia? Curr Opin Psychiatry Mar 2008;21(2):140-150.

72. Habets $P$, Marcelis $M$, Gronenschild E, Drukker M, van Os J. Reduced cortical thickness as an outcome of differential sensitivity to environmental risks in schizophrenia. Biological psychiatry Mar 1 2010;69(5):487-494.

73. Bell RP, Foxe JJ, Nierenberg J, Hoptman MJ, Garavan H. Assessing white matter integrity as a function of abstinence duration in former cocaine-dependent individuals. Drug Alcohol Depend Apr 1 2011;114(2-3):159-168.

74. Bora E, Yucel M, Fornito A, Pantelis C, Harrison BJ, Cocchi L, Pell G, Lubman DI. White matter microstructure in opiate addiction. Addict Biol Jan 2012;17(1):141-148.

75. Lane SD, Steinberg JL, Ma L, Hasan KM, Kramer LA, Zuniga EA, Narayana PA, Moeller FG. Diffusion tensor imaging and decision making in cocaine dependence. PLoS One 2010;5(7):e11591.

76. Kanaan RA, Allin M, Picchioni M, Barker GJ, Daly E, Shergill SS, Woolley J, McGuire PK. Gender differences in white matter microstructure. PLoS One 2012;7(6):e38272.

77. Menzler K, Belke M, Wehrmann E, et al. Men and women are different: diffusion tensor imaging reveals sexual dimorphism in the microstructure of the thalamus, corpus callosum and cingulum. Neurolmage Feb 14 2011;54(4):2557-2562.

78. Sullivan EV, Adalsteinsson E, Hedehus M, Ju C, Moseley M, Lim KO, Pfefferbaum A. Equivalent disruption of regional white matter microstructure in ageing healthy men and women. Neuroreport Jan 22 2001;12(1):99-104.

79. Price G, Cercignani M, Parker GJ, Altmann DR, Barnes TR, Barker GJ, Joyce EM, Ron MA. Abnormal brain connectivity in first-episode psychosis: a diffusion MRI tractography study of the corpus callosum. Neurolmage Apr 1 2007;35(2):458-466.

80. Rametti G, Junque C, Falcon C, Bargallo N, Catalan R, Penades R, Garzon B, Bernardo M. A voxel-based diffusion tensor imaging study of temporal white matter in patients with schizophrenia. Psychiatry Research Mar 31 2009;171(3):166-176.

81. Liao $Y$, Huang $X, W u Q$, et al. Is depression a disconnection syndrome? Meta-analysis of diffusion tensor imaging studies in patients with MDD. Journal of psychiatry \& neuroscience : JPN Jun 12 2012;37(4):110180.

82. Jones DK, Cercignani M. Twenty-five pitfalls in the analysis of diffusion MRI data. NMR in biomedicine Aug 2010;23(7):803-820.

83. Kyriakopoulos M, Bargiotas T, Barker GJ, Frangou S. Diffusion tensor imaging in schizophrenia. European Psychiatry: the Journal of the Association of European Psychiatrists 2008;23(4):255273. 



\title{
Chapter 3
}

Differential time-course of microstructural white matter in patients with psychotic disorder and individuals at risk: A 3-year follow-up study

\author{
Patrick Domen ${ }^{1}$ \\ Sanne Peeters ${ }^{1,5}$ \\ Stijn Michielse ${ }^{1}$ \\ Ed Gronenschild ${ }^{1}$ \\ Wolfgang Viechtbauer ${ }^{1}$ \\ Alard Roebroeck ${ }^{3}$ \\ Jim van $\mathrm{Os}^{1,2}$ \\ Machteld Marcelis ${ }^{1,4}$
}

for Genetic Risk and Outcome of Psychosis (G.R.O.U.P). 
${ }^{1}$ Department of Psychiatry and Neuropsychology, School for Mental Health and Neuroscience, Maastricht University, Maastricht, The Netherlands.

${ }^{2}$ King's College London, King's Health Partners, Department of Psychosis Studies, Institute of Psychiatry, London, United Kingdom.

${ }^{3}$ Department of Cognitive Neuroscience, Maastricht University, Maastricht, The Netherlands.

${ }^{4}$ Institute for Mental Health Care Eindhoven (GGzE), Eindhoven, The Netherlands.

${ }^{5}$ Faculty of Psychology and Educational Sciences, Open University of the Netherlands, Heerlen, the Netherlands.

Published as:

Domen P, Peeters S, Michielse S, et al. Differential Time Course of Microstructural White Matter in Patients With Psychotic Disorder and Individuals at Risk: A 3-Year Follow-up Study. Schizophrenia Bulletin.2017;43:160-170. 


\section{ABSTRACT}

Background. Although widespread reduced white matter integrity is a consistent finding in cross-sectional DTI studies of schizophrenia, little is known about the course of these alterations. This study examined to what degree microstructural white matter alterations display differential trajectories over time as a function of level of psychosis liability.

Methods. Two DTI scans with a 3-year time interval were acquired from 159 participants ( 55 patients with a psychotic disorder, 55 non-psychotic siblings and 49 healthy controls) and processed with tract-based spatial statistics (TBSS). The mean FA change over time was calculated. Main effects of group, as well as group $\times$ region interactions in the model of FA change were examined with multilevel (mixed-effects) models.

Results. Siblings revealed a significant mean FA decrease over time compared to controls $(B=-0.004, p=0.04)$, resulting in a significant sibling-control difference at followup $(B=-0.007, p=0.03)$. Patients did not show a significant change over time, but their mean FA was lower than controls both at baseline and at follow-up. A significant group $\times$ region interaction $\left(\chi^{2}: 105.4, p=0.01\right)$ revealed group differences in FA change in the right cingulum, left posterior thalamic radiation, right retrolenticular part of the internal capsule and the right posterior corona radiata.

Conclusion. Whole brain mean FA remained stable over a 3-year period in patients with psychotic disorder and declined over time in non-affected siblings, so that at follow-up both groups had lower FA with respect to controls. The results suggest that liability for psychosis may involve a process of white matter alterations. 


\section{Introduction}

The functional dysconnectivity hypothesis of schizophrenia suggests that symptoms originate as a result of miscommunication between different brain areas ${ }^{1,2}$, associated with microstructural white matter (WM) alterations. Diffusion tensor imaging (DTI) is used to identify potential WM microstructural correlates. Fractional anisotropy (FA) combines information on myelination, fiber density and number of axons in one measure, whereas additional diffusion parameters (axial-, radial-, and mean diffusivity) may provide more specific information on white matter integrity ${ }^{3}$. A large number of cross-sectional DTI studies of patients in early and later stages of schizophrenia have shown decreased FA with respect to controls in several white matter tracts throughout the brain ${ }^{4,5}$. However, it is not clear when these WM alterations occur and how they develop over time ${ }^{6}$. Both a neurodevelopmental model ${ }^{7,8}$, implying that white matter alterations are present before disease onset and a post-onset progression model, indicating that structural brain abnormalities progress over time after disease onset, have been proposed ${ }^{9,10}$. In support of the neurodevelopmental model, several cross-sectional DTI studies have revealed microstructural WM alterations in frontotemporal and -parietal connections in first-degree relatives without symptoms ${ }^{11,12}$, in clinical high-risk populations before onset ${ }^{13,14}$, and in patients with early onset psychosis ${ }^{15}$. Indeed, the lifetime trajectory of WM alterations in schizophrenia suggests higher percentages of WM loss in the first years of the illness, implicating altered neurodevelopment ${ }^{6,16}$. The literature on longitudinal WM changes, however, is too limited to draw firm conclusions on neurodevelopmental or progressive changes, as well as the role of medication ${ }^{16}$. A study by Carletti and colleagues (2012) showed a significant progressive reduction in FA in the left frontal white matter in individuals at 'Ultra-High Risk' (UHR) who developed psychosis compared to UHR subjects who did not make a 'transition' ${ }^{17}$. Reis Marques and colleagues (2014) found an increase in FA in first episode patients (both responders and non-responders to AP medication) over a 12 -week period ${ }^{18}$, and Garver and colleagues (2008) reported reduced mean diffusivity after 28 days of treatment in drug-responding patients with schizophrenia, which was not found in non-responders ${ }^{19}$. A fourth study compared WM FA changes between individuals during a more chronic course of schizophrenia $(n=49)$ and healthy controls $(n=16)$, reporting a stronger FA decline over 4 years in frontal, temporal, and parietal WM in the controls. A subgroup of patients with poor outcome could be differentiated from a group with good outcome by regional progression of WM alterations in an adjacent precentral/postcentral area ${ }^{20}$.

Not only DTI, but also longitudinal volumetric studies are scarce. Decreases over time have been described in recent onset and first-episode patients in frontal lobe white matter volume ${ }^{21}$ and temporal lobe WM volume, respectively ${ }^{22}$. However, in patients 
aged up to 51 years, van Haren and colleagues (2008) described an abnormal curved trajectory of volume change, with normalization of age-related volume change later in life ${ }^{23}$.

In our recent cross-sectional diffusion analyses of patients with a psychotic disorder, siblings and controls $(n=258)$, patient-specific microstructural WM alterations were found in the corpus callosum and other WM tracts ${ }^{24}$. This sample was re-scanned approximately 3 years later to examine whether the evolution of FA over time varies as a function of familial risk for psychotic disorder.

\section{Methods}

\section{Participants}

Participants were recruited in the context of a multicentre longitudinal study (Genetic Risk and Outcome of Psychosis, G.R.O.U.P.) in the Netherlands ${ }^{25}$. The MRI add-on study was conducted in Maastricht, the Netherlands (see Domen and colleagues ${ }^{24}$ or the Supplemental Method section for full information on in- and exclusion criteria of the participants and diagnostic assessments). For the baseline MRI study, 300 participants were included of which 258 provided a valid DTI scan: 85 patients with a psychotic disorder, 93 siblings without a psychotic disorder, and 80 healthy controls. At follow-up, approximately three years later (mean: 3.3 year), a second DTI scan was acquired from 180 participants (loss to follow-up of $40 \%$ ). The final sample comprised 159 participants (55 patients with a psychotic disorder, 55 siblings without a psychotic disorder, and 49 healthy controls), for which a pair of DTI scans was available for longitudinal analysis (Table 1). 
Table 1. Demographic Characteristics of the Participants ( $n=159)$

\begin{tabular}{|c|c|c|c|c|c|c|}
\hline \multirow[b]{2}{*}{ Time point } & \multicolumn{2}{|c|}{ Controls ( $n=49$ ) } & \multicolumn{2}{|c|}{ Siblings $(n=55)$} & \multicolumn{2}{|c|}{ Patients $(n=55)$} \\
\hline & 0 & 1 & 0 & 1 & 0 & 1 \\
\hline Scan interval (days) & \multicolumn{2}{|c|}{$1222 \pm 198$} & \multicolumn{2}{|c|}{$1177 \pm 101$} & \multicolumn{2}{|c|}{$1196 \pm 121$} \\
\hline Age at scan (years) & $31.0 \pm 11.0$ & $34.4 \pm 10.9$ & $30.9 \pm 8.5$ & $34.1 \pm 8.5$ & $28.7 \pm 6.3$ & $32.0 \pm 6.2$ \\
\hline Sex Male (\%) & \multicolumn{2}{|c|}{$19(39 \%)$} & \multicolumn{2}{|c|}{$29(53 \%)$} & \multicolumn{2}{|c|}{$40(73 \%)$} \\
\hline Handedness & \multicolumn{2}{|c|}{83.2} & \multicolumn{2}{|c|}{78.0} & \multicolumn{2}{|c|}{72.2} \\
\hline Level of education & \multicolumn{2}{|c|}{$5.4 \pm 2.0$} & \multicolumn{2}{|c|}{$5.4 \pm 2.1$} & \multicolumn{2}{|c|}{$4.5 \pm 1.9$} \\
\hline Age of onset (years) & \multicolumn{2}{|c|}{-} & \multicolumn{2}{|c|}{-} & \multicolumn{2}{|c|}{$21.8 \pm 6.3$} \\
\hline Illness duration (years) & \multicolumn{2}{|c|}{-} & \multicolumn{2}{|c|}{-} & $5.7 \pm 3.5$ & $10.3 \pm 4.0$ \\
\hline \multirow[t]{2}{*}{ AP medication } & \multirow{2}{*}{\multicolumn{2}{|c|}{-}} & \multirow{2}{*}{\multicolumn{2}{|c|}{-}} & $6693 \pm$ & $5335 \pm$ \\
\hline & & & & & $6254^{\mathrm{a}}$ & $5715^{b}$ \\
\hline \multicolumn{7}{|l|}{ Diagnosis } \\
\hline Schizophrenia & \multicolumn{2}{|c|}{-} & \multicolumn{2}{|c|}{-} & \multicolumn{2}{|r|}{33} \\
\hline Schizoaffective disorder & \multicolumn{2}{|c|}{-} & \multicolumn{2}{|c|}{-} & \multicolumn{2}{|r|}{16} \\
\hline Psychotic disorder NOS & \multicolumn{2}{|c|}{-} & & 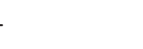 & & 6 \\
\hline $\begin{array}{l}\text { Major depressive } \\
\text { disorder }^{c}\end{array}$ & & 1 & & 5 & & - \\
\hline Substance use & & & & & & \\
\hline Cannabis & & $4.8 \pm 32.0^{d}$ & & $7.1 \pm 49.1^{d}$ & & $36.5 \pm 105.2^{d}$ \\
\hline Other drugs & & 0.0 & & 0.0 & & $15.2 \pm 57.0^{d}$ \\
\hline Alcohol & & $6.3 \pm 10.8^{\mathrm{e}}$ & & $6.0 \pm 5.4^{\mathrm{e}}$ & & $4.9 \pm 7.6^{\mathrm{e}}$ \\
\hline PANSS scores & & & & & & \\
\hline Positive symptoms & $7.3 \pm 1.2$ & $7.4 \pm 0.8$ & $7.4 \pm 0.9$ & $7.4 \pm 0.7$ & $9.6 \pm 3.7$ & $11.6 \pm 5.7$ \\
\hline Negative symptoms & $8.2 \pm 1.2$ & $8.0 \pm 0.2$ & $8.2 \pm 1.3$ & $8.0 \pm 0.3$ & $11.4 \pm 5.2$ & $11.1 \pm 4.2$ \\
\hline Disorganization & $10.3 \pm 1.5$ & $10.1 \pm 0.3$ & $10.3 \pm 0.6$ & $10.1 \pm 0.4$ & $11.7 \pm 2.6$ & $11.8 \pm 2.5$ \\
\hline Excitement & $8.4 \pm 1.3$ & $8.3 \pm 0.5$ & $8.5 \pm 1.2$ & $8.3 \pm 0.6$ & $9.4 \pm 1.9$ & $9.7 \pm 2.5$ \\
\hline Emotional Distress & $9.3 \pm 2.1$ & $9.4 \pm 1.7$ & $9.7 \pm 2.2$ & $9.7 \pm 2.2$ & $12.9 \pm 5.2$ & $14.1 \pm 5.0$ \\
\hline Remission (percentage) & - & - & - & - & $61 \%$ & $62 \%$ \\
\hline
\end{tabular}

Means \pm SDs are reported. AP, Antipsychotics; NOS, not otherwise specified; PANSS, Positive and Negative Syndrome Scale.

${ }^{\text {a }}$ Cumulative exposure (in haloperidol equivalents), lifetime until baseline assessment

${ }^{\mathrm{b}}$ Exposure (in haloperidol equivalents) over last 3 years

${ }^{\mathrm{C}}$ History of major depressive disorder, no current episodes at baseline or in last three years

${ }^{\mathrm{d}}$ Mean number of times; last 12 months

e Weekly consumptions last 12 months 
The sample included 129 families of which 16 families contributed one patient and one healthy sibling and 1 family contributed one patient and two healthy siblings. Six families contributed two healthy siblings, one family contributed three healthy siblings and four families contributed two healthy controls. In addition, 38 families contributed a single patient, 22 families contributed a single sibling, and 41 families contributed a single control.

The standing ethics committee approved the study protocol, and all participants gave written informed consent in accordance with the committee's guidelines.

\section{Measures}

\section{Symptoms}

At both time points, symptoms were assessed with the Positive and Negative Syndrome Scale (PANSS) ${ }^{26}$. The five factor model by van der Gaag and colleagues (2006) was used, dividing the PANSS in positive symptoms, negative symptoms, disorganization symptoms, excitement, and emotional distress ${ }^{27}$. The scores of the individual items of the 5 symptom dimensions were summed. To assess clinical remission, the operationalized criteria described by Andreasen and colleagues (2005) ${ }^{28}$ were applied.

Educational level (at baseline) was defined as the highest accomplished level of education. Handedness was assessed using the Annett Handedness Scale ${ }^{29}$.

\section{Medication}

In the patient group, antipsychotic (AP) medication use was determined by patient report and verified with the treating consultant psychiatrist. Best estimate lifetime (cumulative) AP use at baseline was determined by multiplying the number of days of AP use with the corresponding haloperidol equivalents and summing these scores for all periods of AP use (including the exposure period between baseline assessment for the G.R.O.U.P. study and the moment of baseline MRI scanning), using the published converting formulas for AP dose equivalents described by Andreasen and colleagues ${ }^{30}$. The same procedure was used for calculating cumulative AP exposure during the 3-year follow-up period.

\section{Substance use}

Substance use was measured at both time points with the Composite International Diagnostic Interview (CIDI) sections B-J-L ${ }^{31}$. Alcohol use was defined as the reported number of weekly consumptions during the last 12 months. As data on drug use of the last 3 years was not available, cannabis and other drugs was assessed as reported frequency of use during the last 12 months, as well as lifetime use. CIDI frequency 
data on alcohol, lifetime cannabis and other drug use was available at baseline for respectively 158 participants ( $1 \%$ missing data), 155 participants ( $3 \%$ missing data), and 157 participants ( $1 \%$ missing data).

\section{Image acquisition}

Magnetic resonance imaging scans were obtained at Maastricht University, the Netherlands, using an Allegra Magnetom MR (Siemens, Erlangen, Germany) operating at 3.0 Tesla. At both measurement points, microstructural anatomy was examined using diffusion tensor imaging with an echo-planar-imaging sequence (field of view 230 $\times 230 \mathrm{~mm}^{2}$, TR $10800 \mathrm{~ms}$, TE $84 \mathrm{~ms}$, voxel size $1.8 \times 1.8 \times 1.8 \mathrm{~mm}^{3}$, b-value $1000 \mathrm{~s} /$ $\mathrm{mm}^{2}, 85$ slices, no overlap). As a result of a scanner update at the baseline measurement, two DTI sequences were used: one with 76 directions (of which four diffusionunweighted (B0) and 72 diffusion-weighted (B1000)) and one with 81 directions ( $8 \times \mathrm{B} 0$ and $73 \times \mathrm{B} 1000)$. A potential association between the proportion of baseline scans and group was investigated using a Pearson chi-square test. At follow-up, the DTI sequence comprised 81 directions ( $8 \times B 0$ and $73 \times B 1000)$. Total acquisition time of the DTI sequence was 15 minutes.

\section{Diffusion tensor imaging analysis}

DTI data were processed using tract-based spatial statistics (TBSS) v1.2 in FSL 4.1.6 (FMRIB Analysis Group, Oxford, UK, http://www.fmrib.ox.ac.uk/analysis/research/ tbss). First, standard Siemens DICOM files were transformed into compressed NIFTI format using a custom built in-house software named GIANT (General Image ANalysis Tools developed by EG). Raw data were corrected for head movement and eddy currents invoked during scanning. The BO volume was skull-stripped using FSL's Brain Extraction Tool ${ }^{32}$ and this served as a brain mask for all B volumes.

The next step was fitting a diffusion tensor model at each voxel using data output from the brain extraction, diffusion weighted data and gradient directions following a general linear model (FreeSurfer v4.5.0, http://www.freesurfer.net). After tensor fitting (using the DT-Recon script) the process continued working on FA volumes, eroding them slightly. Nonlinear registration aligned each FA volume to $1 \times 1 \times 1 \mathrm{~mm}$ standard FMRIB58_FA space. The standard FMRIB58_FA contains a template derived from high-resolution images of 58 participants in a well-aligned population (both males and females ranging between 20 and 50 years of age) ${ }^{33}$.

After nonlinear transformation of the FA volumes into standard space, two mean FA skeletons were created; i) one based on three groups ( $n=159$ : controls, siblings, patients) for the cross-sectional analysis at follow-up and ii) one based on six groups ( 3 groups $\times 2$ time-points) for the longitudinal analysis. The mean FA skeleton follows the major WM tracts in each individual participant (normalized in MNI152 space) and 
provides a way to compare between (groups of) participants. The FA threshold was set, using visual inspection of the FA skeleton, at a level of 0.25 , to include major WM tracts whilst removing small peripheral tracts that would cause excess interparticipant variability. In addition, this threshold setting avoided inclusion of regions that are likely to be composed of multiple tissue types or fiber orientations. In the final step, a binary skeleton mask was created and used to extract FA values of the individual participants. The Johns Hopkins University International Consortium for Brain Mapping (JHU ICBM)-DTI-81 white-matter atlas labels ${ }^{34}$ were used to label all voxels and assign a specific tract name. If the voxels did not match with the JHU ICBM labels, they were identified using the JHU white-matter tractography atlas ${ }^{35}$.

\section{Statistical analyses}

From the $38 \mathrm{JHU}$ labelled white matter tracts, skeleton mean FA values per participant per time point were extracted and exported to $R$ (version 3.2.0), a free software environment for statistical computing and graphics ${ }^{36}$.

\section{Longitudinal analyses}

Within-group paired t-tests were done to examine the difference in regional mean FA between baseline and follow-up. Subsequently, a mean FA "change" (delta, $\Delta$ per participant per region) was calculated by subtracting mean FA (baseline) from mean FA (follow-up). The data set was transformed from a wide to a hierarchically structured data set, with 38 regional $\triangle F A$ measures (Level 1) nested in subjects (Level 2) who were part of the same families (Level 3). A mixed- effects model was used to examine the model with $\triangle F A$ measures as the dependent variable and scan-interval as additional covariate. In addition, since the outcome represents means based on varying number of voxels (depending on the region), we used a model in which the error variance for a particular observation was inversely weighted by the number of voxels within the corresponding region (i.e., since the variance of a mean is equal to the variance of a single observation - in this case voxel - divided by the number of values used for the averaging). Main effects of group, corrected for age, sex, handedness, level of education and scan-interval, as well as group $\times$ sex and group $\times$ region interactions in the model of $\triangle F A$ were examined. In each of the 38 regions, between-group factor significance was tested. Regions with a significant group effect were examined with pairwise comparisons (i.e., it was tested whether $\Delta F A$ differed between patients and controls, between siblings and controls, and between patients and siblings).

Due to the large number of regions (and hence tests), Simes' procedure ${ }^{37,38}$ was used to control the false discovery rate when testing the regional within-group mean FA differences between baseline and follow-up, the between-group effects within the 38 regions and the pairwise comparisons. 
Sensitivity analyses were performed with last year cannabis use, lifetime cannabis use and scan type (76 or 81 directions) as additional covariates in three separate models. Since a subgroup of participants in the control $(n=11)$ and sibling group $(n=15)$ had a history of a depressive disorder (and a number of DTI studies with patients with a major depressive disorder have shown decreased FA in several cortical and subcortical WM tracts ${ }^{39}$ ), additional sensitivity analyses were conducted controlling for history of depression.

\section{AP medication}

In patients only, the association between $\triangle \mathrm{FA}$ and respectively last 3-year and lifetime AP use was examined. These variables were entered both as linear and as factored variables (ie, representing the distribution of scores divided by its tertiles: low, moderate or high AP exposure), allowing visualization of dose-response. Sensitivity analyses were done using patient subgroups (with low, moderate, high AP exposure), ie, the association between group and $\triangle F A$ was examined per AP subgroup to examine whether patients with differential AP exposure would have differential FA change over time in comparison to controls and siblings.

\section{Cross-sectional analyses at follow-up}

To examine group differences at follow-up $(n=159)$, a whole-brain mean FA was computed with the $38 \mathrm{WM}$ regions conform the above-described procedure. Whole-brain FA was the dependent variable and random effects (intercepts) were added for each subject and family, including a priori hypothesized confounding variables age, sex, handedness, and level of education as fixed effects.

\section{Results}

\section{Participant characteristics}

The characteristics of the 159 individuals with a baseline and follow-up scan are displayed in Table 1. The majority of the patients were not in need of inpatient care or intensive treatment, as reflected by the low PANSS scores at both time points and the proportion of patients in remission. At baseline, 54 patients were receiving AP medication with a mean dosage in terms of standard haloperidol equivalents of 5.4 milligrams $(\mathrm{mg})(\mathrm{SD}=3.4)$. At follow-up, 45 patients used AP medication (second generation: $n=42$; first generation: $n=3$ ), with a mean dosage of 4.7 milligrams ( $m g$ ) $(S D=5.1)$. The lifetime cumulative AP exposure was $13079.7 \mathrm{mg}$ (SD = 10977.2).

The proportion of baseline scans with 76 directions did not differ between the groups ( $84 \%$ in controls, $82 \%$ in siblings, and $71 \%$ in patients: $\chi^{2}=3.02, d f=2, P=0.22$ ). 


\section{Whole-brain group differences in FA change over time ( $\triangle \mathrm{FA})$}

The mean $\triangle F A$ revealed a slight increase over time in the controls $(0.001)$ and a decrease over time in the siblings $(-0.003)$ and patients $(-0.0002)$, with the mean FA $(0.504)$ of the siblings at follow-up in between that of the controls (0.509) and the patients (0.502) (Fig. 1). At follow-up, there were more white matter tracts showing a decrease than an increase in patients and siblings ( 27 and 23 of the 38 tracts respectively), whereas in controls the proportion of decreases and increases was balanced (Table 2).

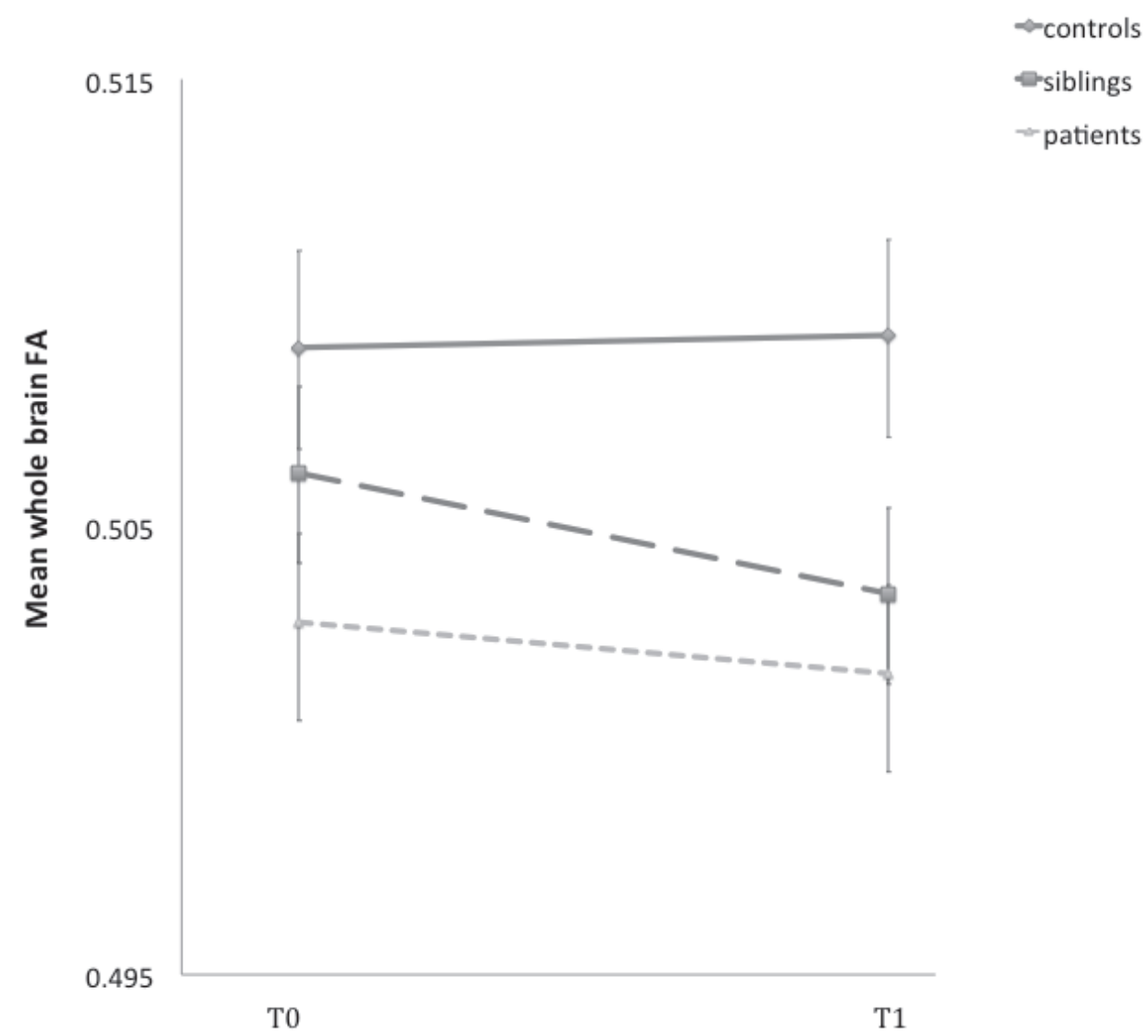

Fig. 1. Group differences in whole brain FA at follow-up. Error bars represent the SE of the mean $\triangle F A$ at baseline and at follow-up. FA, fractional anisotropy 
Table 2. Mean FA per Group at Baseline and at Follow-up

\begin{tabular}{|c|c|c|c|c|c|c|c|c|c|}
\hline \multirow[t]{3}{*}{ Brain region } & \multicolumn{9}{|c|}{ Mean FA } \\
\hline & \multicolumn{3}{|c|}{ Controls } & \multicolumn{3}{|c|}{ Siblings } & \multicolumn{3}{|c|}{ Patients } \\
\hline & TO & $\mathrm{T} 1$ & $\Delta$ & TO & $\mathrm{T} 1$ & $\Delta$ & TO & $\mathrm{T} 1$ & $\Delta$ \\
\hline & .7570 & .7557 & -.0012 & .7551 & .7515 & -.0036 & .7466 & .7446 & -.0020 \\
\hline & .7214 & .7216 & .0003 & .7175 & .7155 & -.0020 & .7035 & .7007 & -.0028 \\
\hline sum & .7877 & .7915 & .0037 & .7891 & .7873 & -.0018 & .7880 & .7897 & .0017 \\
\hline Forceps major & .7004 & .7042 & $.0038^{*}$ & .6974 & .6956 & -.0018 & .6904 & .6896 & -.0009 \\
\hline & .5850 & .5848 & -.0002 & .5827 & .5800 & -.0026 & .5785 & .5779 & -.0006 \\
\hline dy) & .5278 & .5199 & -.0078 & .5305 & .5208 & -.0097 & .4812 & .4855 & .0043 \\
\hline Ant. limb of int. capsule, right & .6311 & .6213 & $-.0098^{*}$ & .6242 & .6154 & $-.0088^{*}$ & .6215 & .6129 & $-.0086^{*}$ \\
\hline Ant. limb of int. capsule, left & .6083 & .6194 & $.0111^{*}$ & .6024 & .6102 & $.0079^{*}$ & .6003 & .6116 & $.0113^{*}$ \\
\hline ost. limb of int. capsule, right & 6949 & .6903 & -.0046 & .6924 & .6859 & $-.0065^{*}$ & .6954 & .6906 & -.0048 \\
\hline le, left & .6934 & .6942 & .0008 & .6917 & .6903 & -.0014 & .6977 & .6953 & -.0024 \\
\hline Retro. part of int. capsule, right & .5807 & .5993 & $.0186^{*}$ & .5819 & .5901 & .0082 & .5834 & .5901 & .0066 \\
\hline Retro. part of int. capsule, left & .5985 & & $-.0102^{*}$ & .5951 & .5859 & $-.0092^{*}$ & .5935 & .5902 & -.0033 \\
\hline nterior corona radiata, right & .5192 & .5119 & -.0073 & .5150 & .5043 & $-.0108^{*}$ & .5056 & .4947 & $-.0110^{*}$ \\
\hline Antorior corona moliata left & .5037 & .5084 & .0047 & .4979 & .4980 & .0002 & .4885 & .4942 & .0057 \\
\hline & .5267 & .5297 & .0030 & .5239 & .5240 & .0001 & .5186 & .5191 & .0006 \\
\hline left & .5319 & .5207 & $-.0111^{*}$ & .5261 & .5169 & $-.0091^{*}$ & .5235 & .5121 & $-.0114^{*}$ \\
\hline Posterior corona radiata, right & .5172 & .5115 & -.0057 & .5094 & .4945 & $-.0150^{*}$ & .5062 & .4873 & $-.0189^{*}$ \\
\hline Deterior coron m riats left & .5010 & .5134 & $.0125^{*}$ & .4892 & .5018 & $.0125^{*}$ & .4835 & .4999 & $.0163^{*}$ \\
\hline ght & .6269 & .6260 & -.0009 & .6207 & .6162 & -.0045 & .6180 & .6081 & -.0099 \\
\hline Post. & 6179 & .6255 & .0076 & .6105 & .6094 & -.0011 & .6011 & .6123 & .0111 \\
\hline um $^{n}$, right & .5732 & .5744 & .0012 & .5687 & .5637 & -.0050 & .5656 & .5635 & -.0020 \\
\hline Sagittal s & .5706 & .5746 & .0040 & .5609 & .5625 & .0016 & .5617 & .5641 & .0024 \\
\hline Exter & .4769 & .4770 & .0001 & .4741 & .4690 & -.0050 & .4711 & .4679 & -.0032 \\
\hline Exterr & .4731 & .4755 & .0024 & .4679 & .4698 & .0020 & .4652 & .4715 & .0063 \\
\hline 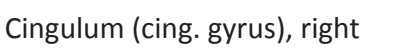 & .6104 & .6403 & $.0299^{*}$ & .6099 & .6333 & $.0233^{*}$ & .6072 & .6378 & $.0305^{*}$ \\
\hline Cingulum (cing. gyrus), left & .6391 & .6048 & $-.0343^{*}$ & .6344 & .6016 & $-.0328^{*}$ & .6344 & .6023 & $-.0320^{*}$ \\
\hline Cingulum (hippoc), right & .5556 & .5585 & .0029 & .5602 & .5393 & $-.0210^{*}$ & .5535 & .5445 & -.0090 \\
\hline Cingulum (hippoc), left & .5486 & .5702 & $.0216^{*}$ & .5421 & .5552 & .0131 & .5417 & .5599 & $.0182^{*}$ \\
\hline terminalis, right & .5581 & .5806 & $.0225^{*}$ & .5547 & .5701 & $.0153^{*}$ & .5454 & .5671 & $.0217^{*}$ \\
\hline Fornix, stria terminalis, left & .5625 & .5562 & -.0063 & .5590 & .5433 & $-.0156^{*}$ & .5509 & .5426 & -.0084 \\
\hline Sup. longitudinal fasc., right & .5298 & .5313 & .0015 & .5325 & .5297 & -.0028 & .5320 & .5241 & -.0079 \\
\hline Sup. longitudinal fasc., left & .5303 & .5243 & -.0060 & .5305 & .5258 & -.0047 & .5271 & .5233 & -.0038 \\
\hline Sup. fronto-occipital fasc.,right & .5380 & .5308 & -.0072 & .5329 & .5281 & -.0048 & .5214 & .5155 & -.0060 \\
\hline
\end{tabular}


Table 2. Mean FA per Group at Baseline and at Follow-up (continued)

\begin{tabular}{lcccccccccc}
\hline Brain region & \multicolumn{1}{c}{ Mean FA } \\
\cline { 2 - 11 } & \multicolumn{3}{c}{ Controls } & \multicolumn{3}{c}{ Siblings } & \multicolumn{3}{c}{ Patients } \\
\cline { 2 - 11 } & T0 & T1 & $\Delta$ & T0 & T1 & $\Delta$ & T0 & T1 & $\Delta$ \\
\hline Sup. fronto-occipital fasc., left & .5276 & .5252 & -.0023 & .5189 & .5188 & -.0001 & .5097 & .5123 & .0026 \\
Uncinate fasciculus, right & .5523 & .5516 & -.0006 & .5393 & .5413 & .0019 & .5395 & .5387 & -.0008 \\
Uncinate fasciculus, left & .5263 & .5179 & -.0084 & .5109 & .5074 & -.0035 & .5071 & .5108 & .0037 \\
Tapetum, right & .6164 & .6121 & -.0042 & .5907 & .5824 & -.0083 & .5910 & .5714 & $-.0196^{*}$ \\
Tapetum, left & .6345 & .6343 & -.0002 & .6106 & .5983 & -.0124 & .5942 & .5812 & -.0130 \\
\hline
\end{tabular}

Note: $\Delta=$ mean FA difference (mean FA follow-up - mean FA baseline), with values in italics representing FA decreases and bold representing FA increases. FA, fractional anisotropy

${ }^{n}$ incl. inferior longitudinal fasciculus and inferior fronto-occipital fasciculus

* Results of a within-group paired t-test examining significant within group $\triangle F A$ differences per brain region $(\mathrm{P}<0.05)$, accounting for a false discovery rate with the Simes' procedure (38 regions $\times 3$ groups) $\left({ }^{*} \mathrm{P}_{\text {simes }}<.003\right)$.

There was no significant association between group and $\Delta \mathrm{FA}\left(\mathrm{x}^{2}: 4.9, \mathrm{df}=2, \mathrm{p}=0.09\right)$. Although the direction of effect for patients compared to controls indicated a decrease in $F A$ over time, the difference was not significant $(B=-0.002, p=0.19)$; additional correction for last year cannabis use, lifetime cannabis use and scan type did not change the results of the patient-control comparisons $(B=-0.002, p=0.22 ; B=-0.002$, $p=0.30$ and $B=-0.002, p=0.17$ respectively). In siblings compared to controls, there was a significant decrease in $F A(B=-0.004, p=0.04)$. This effect remained significant when controlled for scan type $(B=-0.004, p=0.04)$ and last year cannabis use $(B=-$ $0.004, p=0.04)$, and close to significant when controlled for lifetime cannabis use $(B=-0.003, p=0.07)$ and history of depression $(B=-0.003, p=0.06)$. No significant group $\times$ sex interaction in the model of $\Delta F A\left(\chi^{2}=0.9, d f=2, p=0.62\right)$ was found.

\section{Regional group differences in $\triangle \mathrm{FA}$}

There was a significant group $\times$ region interaction in the model of $\triangle F A\left(\chi^{2}: 105.4\right.$, $d f=74, p=0.01$ ), indicating that group differences varied as a function of region. The group factor was significant in 6 of the 38 regions, of which four regions survived after Simes' correction. Patients had a significant smaller FA increase in the right retrolenticular part of the internal capsule (RPIC), and a decrease in the right cingulum and the right posterior corona radiate (PCR) compared to controls. Siblings had a significant smaller FA increase in the right RPIC and a decrease in the right cingulum compared to controls. In comparison to patients and controls, siblings had a decrease in the left posterior thalamic radiation (PTR) (Table 3, Fig. 2). 


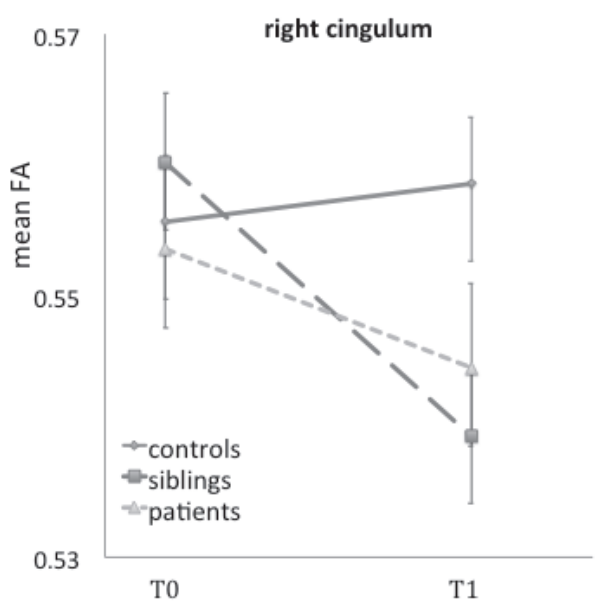

right posterior corona radiata

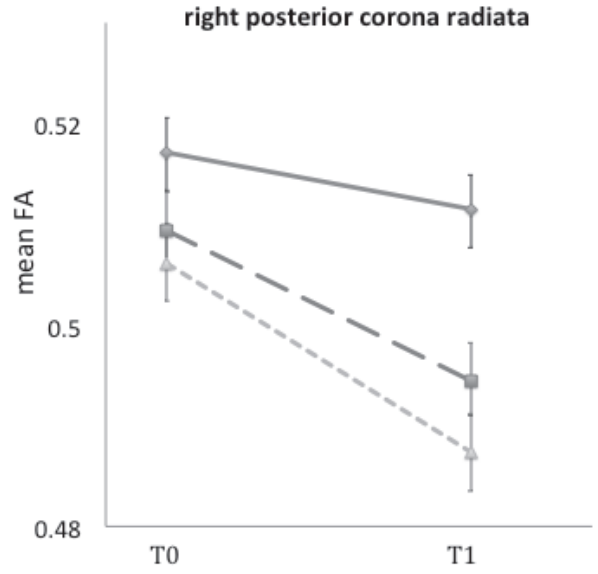

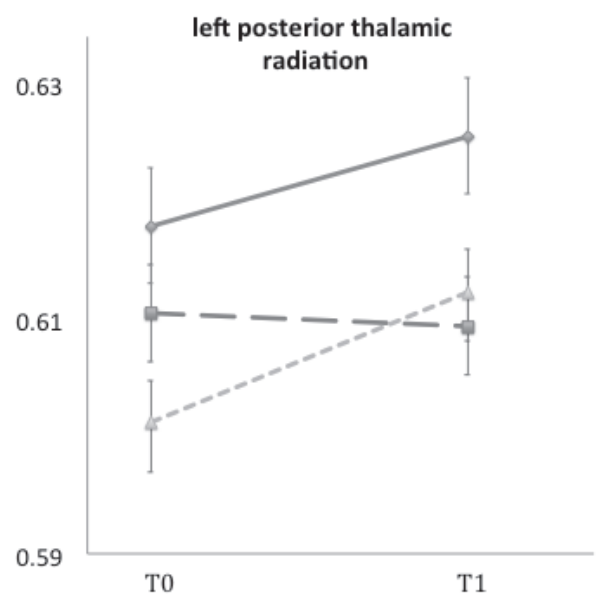

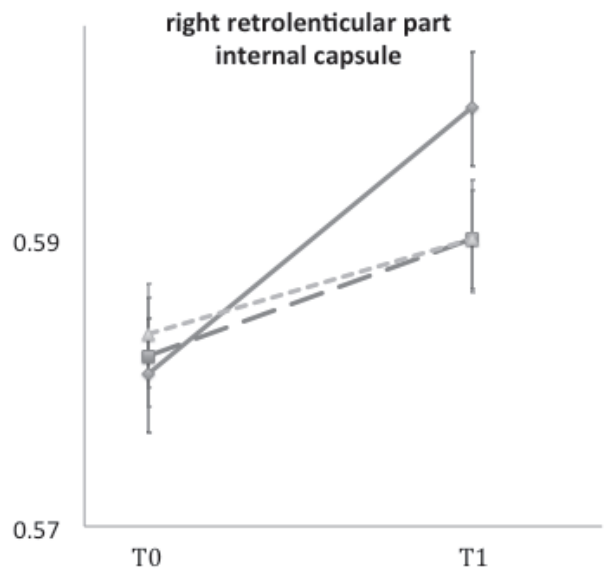

Fig. 2. Group $\times$ region interactions in the model of $\triangle F A$. Group differences in mean $\triangle F A$ in four WM tracts are displayed $\left(\mathrm{P}_{\text {Simes }}<.02\right)$, corresponding with table 3 . Error bars represent the SE of the mean $\triangle F A$ at baseline and at follow-up. FA, fractional anisotropy 
Table 3. Group Comparisons in Regions with a Significant Group Effect

\begin{tabular}{lcccc}
\hline Brain Region & $\begin{array}{c}\text { Between- } \\
\text { group effect }\end{array}$ & $\begin{array}{c}\text { Patient- } \\
\text { Control }\end{array}$ & $\begin{array}{c}\text { Sibling- } \\
\text { Control }\end{array}$ & $\begin{array}{c}\text { Patient- } \\
\text { Sibling }\end{array}$ \\
\cline { 2 - 5 }$\left(\chi^{2}, \mathrm{df}=2, \mathrm{P}\right)$ & $(\mathrm{B}, \mathrm{P})$ & $(\mathrm{B}, \mathrm{P})$ & $(\mathrm{B}, \mathrm{P})$ \\
\hline RPIC, right & $11.8,0.003^{\mathrm{a}}$ & $-0.01,0.001^{*}$ & $-0.01,0.008^{*}$ & $-0.003,0.49$ \\
Posterior corona radiata, right & $10.5,0.005^{\mathrm{a}}$ & $-0.01,0.001^{*}$ & $-0.009,0.03$ & $-0.004,0.29$ \\
Posterior thalamic radiation, right & $9.0,0.01$ & $-0.01,0.003$ & $-0.004,0.27$ & $-0.007,0.05$ \\
Posterior thalamic radiation, left & $14.2,0.0008^{\mathrm{a}}$ & $0.004,0.31$ & $-0.009,0.01^{*}$ & $0.01,0.0003^{*}$ \\
Cingulum, right & $19.6,0.0001^{\mathrm{a}}$ & $-0.01,0.01^{*}$ & $-0.02,9.7 \times 10^{-6^{*}}$ & $0.01,0.05$ \\
Superior longitudinal fasciculus, right & $9.4,0.009$ & $-0.01,0.002$ & $-0.004,0.15$ & $-0.005,0.08$ \\
\hline
\end{tabular}

Note: The $\chi^{2}$ and the P-values represent the significant results of the between-group factor analyses per region in multilevel modelling $\left({ }^{\mathrm{a}} \mathrm{P}_{\text {simes }}<.005\right)$. B and $\mathrm{P}$-values of the individual group comparisons are shown $\left({ }^{*} \mathrm{P}_{\text {simes }}<.02\right)$. Analyses are controlled for age, sex, handedness, level of education, and scan interval. RPIC, Retrolenticular part of internal capsule

\section{Antipsychotic (AP) medication}

Within-patients analyses showed that there was a close to significant association between lifetime AP use (linear) and whole-brain mean $\triangle F A\left(B=-2.5 \times 10^{-7}, p=0.08\right)$. Compared to low lifetime AP exposure, patients with high AP exposure showed significantly more FA decrease over time $(B=-0.008, p=0.04)$, which was not the case for patients with moderate exposure $(B=-0.005, p=0.15)$. With regard to AP exposure over the last three years, a significant negative association was found between cumulative AP exposure (linear) and whole brain mean $\triangle F A$ over the last three years $(B=-5.6 \times$ $10^{-7}, p=0.01$ ). Higher levels of AP medication over the last three years predicted a stronger decrease in FA: moderate vs. low exposure: $B=-0.006, p=0.04$; high vs. low exposure $(B=-0.009, p=0.004)$.

The group analyses based on AP medication subgroups (low, moderate, high cumulative AP medication exposure) showed that there was a significant decrease in FA over time in patients with the highest level of AP exposure (lifetime and over the last three years) compared to controls, but not in patients with moderate or low AP exposure. Siblings showed a significant stronger decrease in FA than patients with the lowest AP exposure (See supplemental Table 1).

\section{Whole-brain group differences in mean FA at follow-up}

There was a significant association between group and $F A$ at follow-up $\left(\chi^{2}: 10.0, d f=2\right.$, $p=0.007)$ : siblings $(B=-0.007, p=0.03)$ and patients $(B=-0.010, p=0.005)$ showed a significantly lower mean FA compared to the controls. The sibling - patient comparison was neither large nor significant ( $B=-0.001, p=0.46)$ (Fig. 1). 
Supplemental Table 1. Associations between group and $\triangle F A$ based on AP medication subgroups

\begin{tabular}{|c|c|c|c|c|}
\hline Antipsychotics & $\begin{array}{c}\text { Between-group } \\
\text { effect } \\
\left(\chi^{2}, d f=2, P\right)\end{array}$ & Patient-Control & Sibling-Control & Sibling-Patient \\
\hline \multicolumn{5}{|l|}{ Lifetime exposure } \\
\hline $\operatorname{Low}(n=15)$ & $6.1,0.05$ & $-0.0009,0.66$ & $-0.004,0.03^{*}$ & $-0.003,0.16$ \\
\hline Moderate $(n=15)$ & $5.2,0.08$ & $-0.003,0.22$ & $-0.003,0.04^{*}$ & $-0.001,0.66$ \\
\hline High ( $n=15)$ & $8.7,0.01$ & $-0.005,0.02^{*}$ & $-0.004,0.03^{*}$ & $0.001,0.46$ \\
\hline \multicolumn{5}{|l|}{ Last 3-years exposure } \\
\hline Low $(n=18)$ & $9.9,0.007$ & $0.002,0.34$ & $-0.004,0.04^{*}$ & $-0.006,0.007^{*}$ \\
\hline Moderate $(n=18)$ & $6.5,0.04$ & $-0.004,0.10$ & $-0.004,0.04^{*}$ & $0.0002,0.93$ \\
\hline High $(n=17)$ & $13.8,0.001$ & $-0.007,0.004^{*}$ & $-0.004,0.03^{*}$ & $0.004,0.07$ \\
\hline
\end{tabular}

Results from the association between group status and $\triangle \mathrm{FA}$, using patient subgroups (with low, moderate, high AP exposure). The $\chi^{2}$ and the P-values represent the significant results of the between-group factor analyses $(P<0.05)$. Individual group comparisons are shown $(B, P$-value, $P<0.05)$. Analyses are controlled for age, sex, handedness, level of education, and scan interval.

\section{Discussion}

This longitudinal DTI study showed a relatively stable whole-brain mean FA course in patients with schizophrenia after the critical phase (ie, $>5$ years of illness duration), whereas a significant decline in mean FA was observed in siblings. The effect of group varied as a function of region, as indicated by a significant group by region interaction. Overall, there were more tracts showing a decrease than an increase over time in both patients and siblings, with a significant group difference in four white matter tracts.

\section{Findings in patients}

The study showed that, over a three-year period, the overall mean FA remained stable in patients, being continuously lower than the mean FA of controls and siblings. This data is partly in line with the results of the study by Mitelman and colleagues (2009), examining an older (than the present) sample (of \pm 41 years) of patients with a diagnosis of schizophrenia and healthy controls, also showing a rather stable FA in patients, and a greater, probably normal age-dependent, FA decline over time in frontal, temporal and parietal white matter in healthy controls ${ }^{20}$.

The results suggest that major white matter alterations in patients may have occurred in the early stages of the illness, as described previously ${ }^{15}$, without ongoing progression. Alternatively, WM may have been modulated by AP medication, which was supported in the current study by the small but significant negative within-group 
effect of AP medication on $\triangle \mathrm{FA}$, with a dose-response effect for AP exposure over the last three years. Moreover, patients with the highest AP exposure levels (lifetime, as well as over the last three years) had a significant decrease in FA over time compared to controls, whereas this was not the case for patients with low or moderate AP exposure compared to controls.

Together with the widespread microstructural WM alterations presented in cross-sectional studies of at-risk populations and first episode psychotic patients ${ }^{5}$, the data add to the evidence for an early developmental origin of WM alterations in schizophrenia

${ }^{8}$. Indeed, a significant progressive reduction in FA of the left frontal white matter has been found in UHR subjects who later developed psychosis compared to UHR subjects who did not make the transition ${ }^{17}$.

Despite the overall mean FA being constant, most WM tracts in the three groups showed minor (non-significant) changes in individual FA trajectories (Table 2). White matter tracts develop at different ages in different curvilinear patterns (inverted U-shape), with FA increases from the newborn period to adolescence, shows decelerated maturation until mid-adulthood and subsequently displays more rapid decline during old age ${ }^{40,41}$. The WM tracts of the controls in this study may be at the top of the normal age trajectory curve because of the proportionally equal number of WM tracts with an FA increase and decrease. In contrast, patients showed a higher number of WM tracts with FA decreases, suggesting an altered developmental pattern, i.e., an earlier than expected age-related decrease of WM. Specifically, a smaller FA increase in the RPIC and decrease in the PCR and the cingulum (all right-sided) was found in patients. These tracts have face-validity, as they are frequently described in relation to fronto-temporal disconnection in schizophrenia ${ }^{4,42}$.

\section{Findings in siblings}

This is the first longitudinal study showing whole-brain white matter alterations over time in a sample of healthy participants at higher than average risk for psychotic disorder (siblings of patients with psychotic disorder). At baseline, there was no significant difference in whole-brain mean FA between siblings and controls, whereas such a difference was apparent between siblings and patients ${ }^{24}$. At follow-up, siblings showed a significant lower whole-brain mean FA with respect to the controls and the significant difference with patients was no longer apparent. The difference at followup was confirmed by a significant FA decrease over time in siblings compared to controls, which was not observed in patients. At the level of individual tracts, almost $2 / 3^{\text {rd }}$ of the WM tracts in siblings revealed a decrease in FA over time, compared to the more balanced ratio of FA increases and decreases in the controls. Specifically, the left PTR and the right cingulum revealed a significant decrease of mean FA over time in siblings, whereas controls showed an increase. The PTR has previously been 
associated with schizophrenia ${ }^{15,43}$, e.g. with impaired emotional self-awareness ${ }^{44}$. To our knowledge subtle FA decreases in the PTR have only been associated with bipolar ${ }^{45}$ but not psychosis liability. As mentioned before, all WM tracts have different maturational trajectories ${ }^{46}$. The cingulum has one of the most prolonged maturation periods and reaches its peak FA only after 40 years of age ${ }^{47}$. Given the mean age of 34 years in the siblings and of 32 years in the patients, the earlier than expected FA decline may reflect disturbed WM maturation from an early age, suggesting a neurodevelopmental origin. Alternatively, it may suggest progression associated with illness vulnerability, though the findings in the patients did not support this.

Decreased anisotropy in the cingulum has also been described in several crosssectional studies in patients with schizophrenia ${ }^{48,49}$ and in the longitudinal study of Mitelman and colleagues (2009), where the left anterior cingulate gyrus was one of the two areas that showed a greater decline in FA in patients with schizophrenia compared to healthy participants ${ }^{20}$. Regarding the present finding of a patient-control difference in this region, current and previous cross-sectional DTI findings ${ }^{50}$ in siblings may be suggestive of a WM intermediate phenotype.

Clinically, WM alterations in (sub-)regions of the cingulum have been related to impairments in impulsivity ${ }^{51}$ and executive functioning ${ }^{52}$ as well as to positive and negative symptoms ${ }^{53}$ in patients with schizophrenia. As mild cognitive alterations are present in non-affected relatives ${ }^{54,55}$, it may be hypothesized that subclinical expression of symptoms are associated with this white matter intermediate phenotype, which will be the topic of further investigation.

\section{Methodological considerations}

Although the present study has several strengths, such as the rather large sample size, the longitudinal design covering a three-year period, and the inclusion of both patients and their healthy siblings, there are some limitations that need to be taken into account when interpreting the results.

AP medication may have an effect on $\mathrm{WM}^{15}$. Until now, only a handful of longitudinal diffusion studies have been published, examining (short-term) effects of AP medication on microstructural white matter (pre-post treatment measurements) ${ }^{56-59}$. The results of the present study were supportive of an effect of (especially the highest) cumulative medication exposure levels on FA change over time, both in within-patients analyses and in between-group analyses based on AP exposure subgroups (with one-third of the patients in each subgroup). However, as the FA change in siblings, who were not using AP medication, was also significantly different from controls, AP exposure may be one of the contributing factors of microstructural white matter alteration in patients with psychotic disorder. 
The same applies to drug use. The present study sample was not drug-free which may have influenced our results. Study results differ with respect to the potential influence of cannabis use on white matter alterations in patients with schizophrenia ${ }^{60}$. Although the significant results in the control-sibling comparison remained stable after controlling for cannabis use (last year), lifetime cannabis use appeared to exert some influence, although the effect size was not affected much.

Given the absence of: i) differences in alcohol consumption across groups, ii) other drug use in the controls and siblings, and iii) effects of other drug use on microstructural white matter in the baseline study ${ }^{24}$, sensitivity analyses for these substances were not considered additional informative.

Inconsistencies in the results of the limited longitudinal studies conducted to date may be due to varying patient samples ${ }^{17,20}$, as well as varying acquisition and analysing techniques. Although Reis Marques and colleagues (2012) used TBSS, their procedures and analyses differed from the present study ${ }^{18}$. Furthermore, limited knowledge is available about the margins of across-session reproducibility errors ${ }^{61,62}$. As this was the first longitudinal DTI study including healthy siblings, we used a wholebrain, voxel-based analyses, given the fact that evidence for differential WM regional time-trajectories is missing to date. The results of the group by region interactions are thus hypothesis-generating and may be of use in future longitudinal studies that examine individual WM tracts within distinct development trajectories. 


\section{References}

1. Friston KJ, Frith CD. Schizophrenia: a disconnection syndrome? Clin Neurosci 1995;3(2):89-97.

2. Pettersson-Yeo W, Allen $P$, Benetti $S$, McGuire $P$, Mechelli A. Dysconnectivity in schizophrenia: where are we now? Neuroscience and biobehavioral reviews Apr 2011;35(5):1110-1124.

3. Pierpaoli C, Barnett A, Pajevic S, Chen R, Penix LR, Virta A, Basser P. Water diffusion changes in Wallerian degeneration and their dependence on white matter architecture. Neurolmage Jun 2001;13(6 Pt 1):1174-1185.

4. Ellison-Wright I, Bullmore E. Meta-analysis of diffusion tensor imaging studies in schizophrenia. Schizophr Res Mar 2009;108(1-3):3-10.

5. Samartzis L, Dima D, Fusar-Poli P, Kyriakopoulos M. White Matter Alterations in Early Stages of Schizophrenia: A Systematic Review of Diffusion Tensor Imaging Studies. J Neuroimaging Jan 14 2014; 24:101-10.

6. Kochunov P, Hong LE. Neurodevelopmental and neurodegenerative models of schizophrenia: white matter at the center stage. Schizophr Bull Jul 2014;40(4):721-728.

7. Pantelis C, Yucel M, Wood SJ, et al. Structural brain imaging evidence for multiple pathological processes at different stages of brain development in schizophrenia. Schizophr Bull Jul 2005;31(3):672-696.

8. Rapoport JL, Giedd JN, Gogtay N. Neurodevelopmental model of schizophrenia: update 2012. Molecular psychiatry Dec 2012;17(12):1228-1238.

9. Andreasen NC. The lifetime trajectory of schizophrenia and the concept of neurodevelopment. Dialogues in clinical neuroscience 2010;12(3):409-415.

10. Meyer-Lindenberg A. Neuroimaging and the question of neurodegeneration in schizophrenia. Progress in neurobiology Dec 2011;95(4):514-516.

11. Clark KA, Nuechterlein KH, Asarnow RF, et al. Mean diffusivity and fractional anisotropy as indicators of disease and genetic liability to schizophrenia. Journal of psychiatric research Jul 2011;45(7):980-988.

12. Knochel C, Oertel-Knochel V, Schonmeyer R, et al. Interhemispheric hypoconnectivity in schizophrenia: Fiber integrity and volume differences of the corpus callosum in patients and unaffected relatives. Neuroimage Jan 16 2012;59(2):926-934.

13. von Hohenberg C.C, Pasternak O, Kubicki M, et al. White Matter Microstructure in Individuals at Clinical High Risk of Psychosis: A Whole-Brain Diffusion Tensor Imaging Study. Schizophr Bull Jul 2014;40:895-903.

14. Karlsgodt KH, Niendam TA, Bearden CE, Cannon TD. White matter integrity and prediction of social and role functioning in subjects at ultra-high risk for psychosis. Biol Psychiatry Sep 15 2009;66(6):562-569.

15. Peters BD, Blaas J, de Haan L. Diffusion tensor imaging in the early phase of schizophrenia: what have we learned? Journal of psychiatric research Nov 2010;44(15):993-1004.

16. Canu E, Agosta F, Filippi M. A selective review of structural connectivity abnormalities of schizophrenic patients at different stages of the disease. Schizophr Res Jan 2015;161(1):19-28.

17. Carletti F, Woolley JB, Bhattacharyya S, et al. Alterations in white matter evident before the onset of psychosis. Schizophr Bull Nov 2012;38(6):1170-1179.

18. Reis Marques $\mathrm{T}$, Taylor $\mathrm{H}$, Chaddock $\mathrm{C}$, et al. White matter integrity as a predictor of response to treatment in first episode psychosis. Brain : a journal of neurology Jan 2014;137(Pt 1):172182. 
19. Garver DL, Holcomb JA, Christensen JD. Compromised myelin integrity during psychosis with repair during remission in drug-responding schizophrenia. The international journal of neuropsychopharmacology / official scientific journal of the Collegium Internationale Neuropsychopharmacologicum Feb 2008;11(1):49-61.

20. Mitelman SA, Canfield EL, Newmark RE, et al. Longitudinal Assessment of Gray and White Matter in Chronic Schizophrenia: A Combined Diffusion-Tensor and Structural Magnetic Resonance Imaging Study. The open neuroimaging journal 2009;3:31-47.

21. Ho BC, Andreasen NC, Nopoulos P, Arndt S, Magnotta V, Flaum M. Progressive structural brain abnormalities and their relationship to clinical outcome: a longitudinal magnetic resonance imaging study early in schizophrenia. Arch Gen Psychiatry Jun 2003;60(6):585-594.

22. Whitford TJ, Grieve SM, Farrow TF, Gomes L, Brennan J, Harris AW, Gordon E, Williams LM. Volumetric white matter abnormalities in first-episode schizophrenia: a longitudinal, tensorbased morphometry study. Am J Psychiatry Jul 2007;164(7):1082-1089.

23. van Haren NE, Hulshoff Pol HE, Schnack HG, Cahn W, Brans R, Carati I, Rais M, Kahn RS. Progressive brain volume loss in schizophrenia over the course of the illness: evidence of maturational abnormalities in early adulthood. Biol Psychiatry Jan 1 2008;63(1):106-113.

24. Domen PA, Michielse S, Gronenschild E, Habets P, Roebroeck A, Schruers K, van Os J, Marcelis M. Microstructural white matter alterations in psychotic disorder: A family-based diffusion tensor imaging study. Schizophrenia Research May 2013;146(1-3):291-300.

25. Korver N, Quee PJ, Boos HB, Simons CJ, de Haan L. Genetic Risk and Outcome of Psychosis (GROUP), a multi-site longitudinal cohort study focused on gene-environment interaction: objectives, sample characteristics, recruitment and assessment methods. Int J Methods Psychiatr Res Sep 2012;21(3):205-221.

26. Kay SR, Fiszbein A, Opler LA. The positive and negative syndrome scale (PANSS) for schizophrenia. Schizophr Bull 1987;13(2):261-276.

27. van der Gaag M, Hoffman $T$, Remijsen $M$, et al. The five-factor model of the Positive and Negative Syndrome Scale II: a ten-fold cross-validation of a revised model. Schizophrenia Research Jul 2006;85(1-3):280-287.

28. Andreasen NC, Carpenter WT, Jr., Kane JM, Lasser RA, Marder SR, Weinberger DR. Remission in schizophrenia: proposed criteria and rationale for consensus. Am J Psychiatry Mar 2005;162(3):441-449.

29. Annett M. A classification of hand preference by association analysis. British Journal of Psychology 1970;61(3):303-321.

30. Andreasen NC, Pressler M, Nopoulos P, Miller D, Ho BC. Antipsychotic dose equivalents and dose-years: a standardized method for comparing exposure to different drugs. Biol Psychiatry Feb 1 2010;67(3):255-262.

31. WHO. Composite International Diagnostic Interview (CIDI). 1990(Geneva: World Health Organization).

32. Smith SM. Fast robust automated brain extraction. Hum Brain Mapp Nov 2002;17(3):143-155.

33. Smith SM, Jenkinson $M$, Johansen-Berg $H$, et al. Tract-based spatial statistics: voxelwise anaIysis of multi-subject diffusion data. Neuroimage Jul 15 2006;31(4):1487-1505.

34. Mori S, Oishi $\mathrm{K}$, Jiang $\mathrm{H}$, et al. Stereotaxic white matter atlas based on diffusion tensor imaging in an ICBM template. Neuroimage Apr 1 2008;40(2):570-582.

35. Hua K, Zhang J, Wakana S, et al. Tract probability maps in stereotaxic spaces: analyses of white matter anatomy and tract-specific quantification. Neurolmage Jan 1 2008;39(1):336-347. 
36. $R$ Core Team. $R$ : A language and environment for statistical computing [computer program]. Version 3.2.0. $R$ foundation for Statistical Computing. Vienna, Austria; 2015.

37. Benjamini Y, Hochberg Y. Controlling the false discovery rate: A practical and powerful approach to multiple testing. Journal of the Royal Statistical Society, Series B: Methodological 1995;57:289-300.

38. Simes R. An improved Bonferroni procedure for multiple tests of significance. Biometrika 1986;73:751-754.

39. Liao $\mathrm{Y}$, Huang $\mathrm{X}, \mathrm{Wu} \mathrm{Q}$, et al. Is depression a disconnection syndrome? Meta-analysis of diffusion tensor imaging studies in patients with MDD. J Psychiatry Neurosci 2013;38:49-56.

40. Imperati D, Colcombe S, Kelly C, Di Martino A, Zhou J, Castellanos FX, Milham MP. Differential development of human brain white matter tracts. PloS one 2011;6(8):e23437.

41. Michielse S, Coupland N, Camicioli R, Carter R, Seres P, Sabino J, Malykhin N. Selective effects of aging on brain white matter microstructure: a diffusion tensor imaging tractography study. Neuroimage Oct 1 2010;52(4):1190-1201.

42. Voineskos AN, Lobaugh NJ, Bouix S, et al. Diffusion tensor tractography findings in schizophrenia across the adult lifespan. Brain : a journal of neurology May 2010;133(Pt 5):1494-1504

43. Melicher T, Horacek J, Hlinka J, et al. White matter changes in first episode psychosis and their relation to the size of sample studied: a DTI study. Schizophr Res Mar 2015;162(1-3):22-28.

44. Kubota M, Miyata J, Sasamoto A, et al. Alexithymia and reduced white matter integrity in schizophrenia: a diffusion tensor imaging study on impaired emotional self-awareness. Schizophr Res Nov 2012;141(2-3):137-143.

45. Sprooten E, Brumbaugh MS, Knowles EE, et al. Reduced white matter integrity in sibling pairs discordant for bipolar disorder. Am J Psychiatry Nov 2013;170(11):1317-1325.

46. Yap QJ, Teh I, Fusar-Poli P, Sum MY, Kuswanto C, Sim K. Tracking cerebral white matter changes across the lifespan: insights from diffusion tensor imaging studies. Journal of neural transmission Sep 2013;120(9):1369-1395.

47. Lebel C, Gee M, Camicioli R, Wieler M, Martin W, Beaulieu C. Diffusion tensor imaging of white matter tract evolution over the lifespan. Neuroimage Mar 2012;60(1):340-352.

48. Fujiwara $\mathrm{H}$, Namiki $\mathrm{C}$, Hirao $\mathrm{K}$, et al. Anterior and posterior cingulum abnormalities and their association with psychopathology in schizophrenia: a diffusion tensor imaging study. Schizophr Res Sep 2007;95(1-3):215-222.

49. Wang $\mathrm{L}$, Hosakere $\mathrm{M}$, Trein JC, et al. Abnormalities of cingulate gyrus neuroanatomy in schizophrenia. Schizophr Res Jul 2007;93(1-3):66-78.

50. Arat HE, Chouinard VA, Cohen BM, Lewandowski KE, Ongur D. Diffusion tensor imaging in first degree relatives of schizophrenia and bipolar disorder patients. Schizophr Res Feb 2015;161(2-3):329-339.

51. Hoptman MJ, Ardekani BA, Butler PD, Nierenberg J, Javitt DC, Lim KO. DTI and impulsivity in schizophrenia: a first voxelwise correlational analysis. Neuroreport Nov 15 2004;15(16):24672470.

52. Kubicki $M$, Westin $C F$, Nestor PG, et al. Cingulate fasciculus integrity disruption in schizophrenia: a magnetic resonance diffusion tensor imaging study. Biol Psychiatry Dec 1 2003;54(11):1171-1180.

53. Whitford TJ, Lee SW, Oh JS, et al. Localized abnormalities in the cingulum bundle in patients with schizophrenia: a Diffusion Tensor tractography study. Neurolmage Clinical 2014;5:93-99. 
54. Meijer J, Simons CJ, Quee PJ, Verweij K, Investigators G. Cognitive alterations in patients with non-affective psychotic disorder and their unaffected siblings and parents. Acta psychiatrica Scandinavica Jan 2012;125(1):66-76.

55. Sitskoorn MM, Aleman A, Ebisch SJ, Appels MC, Kahn RS. Cognitive deficits in relatives of patients with schizophrenia: a meta-analysis. Schizophr Res Dec 1 2004;71(2-3):285-295.

56. Ebdrup BH, Raghava JM, Nielsen MO, Rostrup E, Glenthoj B. Frontal fasciculi and psychotic symptoms in antipsychotic-naive patients with schizophrenia before and after 6 weeks of selective dopamine D2/3 receptor blockade. J Psychiatry Neurosci Nov 24 2015;41(1):150030.

57. Ozcelik-Eroglu E, Ertugrul A, Oguz KK, Has AC, Karahan S, Yazici MK. Effect of clozapine on white matter integrity in patients with schizophrenia: a diffusion tensor imaging study. Psychiatry Res Sep 30 2014;223(3):226-235.

58. Szeszko PR, Robinson DG, Ikuta T, Peters BD, Gallego JA, Kane J, Malhotra AK. White matter changes associated with antipsychotic treatment in first-episode psychosis. Neuropsychopharmacology : official publication of the American College of Neuropsychopharmacology May 2014;39(6):1324-1331.

59. Wang $Q$, Cheung $C$, Deng $W$, et al. White-matter microstructure in previously drug-naive patients with schizophrenia after 6 weeks of treatment. Psychol Med Nov 2013;43(11):23012309.

60. DeLisi LE. The effect of cannabis on the brain: can it cause brain anomalies that lead to increased risk for schizophrenia? Curr Opin Psychiatry Mar 2008;21(2):140-150.

61. Jovicich J, Marizzoni M, Bosch B, et al. Multisite longitudinal reliability of tract-based spatial statistics in diffusion tensor imaging of healthy elderly subjects. Neuroimage Nov 1 2014;101:390-403.

62. Vollmar C, O'Muircheartaigh J, Barker GJ, et al. Identical, but not the same: intra-site and inter-site reproducibility of fractional anisotropy measures on two 3.0T scanners. Neuroimage Jul 15 2010;51(4):1384-1394. 



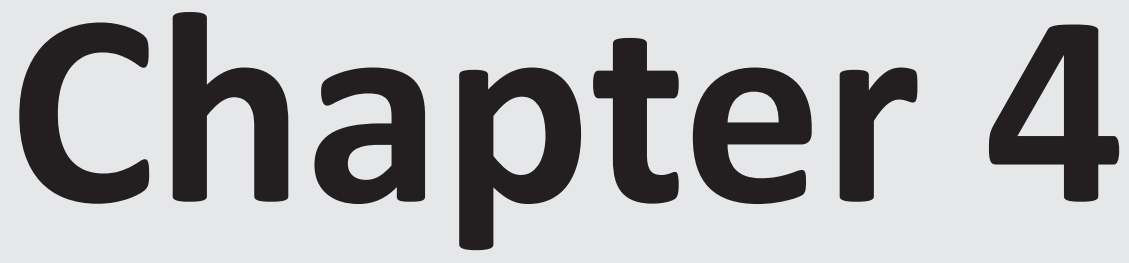

\section{Trauma- and cannabis-associated microstructural white matter changes in patients with psychotic disorder: A longitudinal family-based diffusion imaging study}

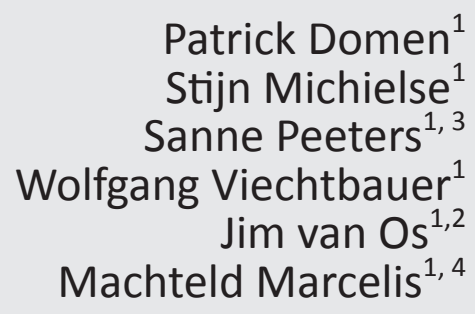

for Genetic Risk and Outcome of Psychosis (G.R.O.U.P.) 
${ }^{1}$ Department of Psychiatry and Neuropsychology, School for Mental Health and Neuroscience, Maastricht University, Maastricht, The Netherlands.

${ }^{2}$ King's College London, King's Health Partners, Department of Psychosis Studies, Institute of Psychiatry, London, United Kingdom.

${ }^{3}$ Faculty of Psychology and Educational Sciences, Open University of the Netherlands, Heerlen, the Netherlands.

${ }^{4}$ Institute for Mental Health Care Eindhoven (GGzE), Eindhoven, The Netherlands. 


\section{Abstract}

Background. Decreased white matter (WM) integrity in patients with psychotic disorder has been a consistent finding in diffusion tensor imaging (DTI) studies. However, most studies are cross-sectional, and do not inform on the time course and the contribution of environmental risk factors on these WM changes, which is what the current study set out to do.

Methods. DTI scans were obtained from 85 patients with a psychotic disorder, 93 non-psychotic siblings and 80 healthy controls, of which $60 \%$ were rescanned 3 years later. In a whole-brain voxel-based analysis, associations between change in fractional anisotropy $(\triangle \mathrm{FA})$ and environmental exposures (cannabis use and childhood trauma exposure) as well as interactions between group and environmental exposure in the model of FA and $\triangle F A$ were investigated. Analyses were adjusted for a priori hypothesized confounding variables: age, sex, and level of education.

Results. At baseline no significant associations were found between FA and both environmental risk factors. At follow-up as well as over a 3-year interval, significant interactions between group and respectively cannabis exposure and trauma exposure in the model of FA and $\triangle F A$ were found. Patients showed more FA decrease over time compared to both controls and siblings when exposed to higher levels of cannabis or childhood trauma.

Conclusion. Higher levels of cannabis or childhood trauma may compromise connectivity over the course of the illness in patients, but not in individuals at low or higher than average genetic risk for psychotic disorder, suggesting interactions between the environment and illness-related factors. 


\section{Introduction}

Reduced fractional anisotropy (FA), widely reported in patients with psychotic disorder ${ }^{1}$, but not in individuals at higher than average genetic risk (siblings of patients) may reflect disease-related dysconnectivity or disease-related differential sensitivity to the environment ${ }^{2,3}$. The risk for psychosis, a condition with adolescent onset, has been related to environmental exposures such as pre- or postnatal birth complications, cannabis use, early life trauma and growing up in an urban environment ${ }^{4}$. These environmental stressors may be the trigger ${ }^{5}$ or the "second hit" ${ }^{6}$, contributing to the emergence of a psychotic illness. However, the potential impact of these environmental risks on white matter (WM) connectivity ${ }^{7,8}$ has not been the subject of detailed investigation in patients with a psychotic disorder. Cross-sectional studies did explore genetic factors, showing a moderate to high heritability of WM FA, ranging from 0.4 $-0.7^{9}$. Also, several candidate genes for WM heritability have been proposed, such as neuregulin1-tyrosine kinase receptor ErbB4 (NRG1-ErbB4), involved in oligodendrocyte, myelin and axonal development and maintenance ${ }^{10}$. Various hypotheses have been postulated, associating environmental risk factors with WM alterations. Cannabis use may induce apoptosis of oligodendrocyte progenitors, affecting WM development ${ }^{11}$. In the literature to date, there is evidence to suggest a negative association between cannabis use and WM volume in patients with schizophrenia ${ }^{12}$, ${ }^{13}$. Results from diffusion tensor imaging (DTI) studies are less clear and have shown increased as well as decreased FA in cannabis-using versus non-using patients with schizophrenia ${ }^{14,15}$. The same ambiguity is seen in samples of non-psychotic substance users compared to non-users, which, on the one hand, showed microstructural WM alterations in specific pathways (corpus callosum and superior longitudinal fascicules) ${ }^{16}$ and the hippocampus (fimbriae), corpus callosum (splenium), commissural fibres ${ }^{17}$, as well as, on the other hand, absence of FA differences ${ }^{18}$.

Studies on early life stress in otherwise healthy children have shown associations between cortisol reactivity and possible alterations in hippocampal and amygdala volumes ${ }^{19}$. Corticosteroids may suppress the final mitosis of glial cells necessary for myelination, influencing WM microstructure ${ }^{20}$. This model is supported by studies showing reduced corpus callosum volume in paediatric inpatients with a history of abuse and neglect ${ }^{21}$ and reduced FA in the left inferior longitudinal fasciculus (ILF) in young adults witnessing domestic violence in childhood ${ }^{22}$. To date, longitudinal diffusion weighted imaging (DWI) studies of patients with schizophrenia are scarce ${ }^{23}$ and apart from studies investigating the effect of medication on WM diffusion measures ${ }^{24,}$ ${ }^{25}$, no study has examined whether traumatic experience has a differential effect on FA over time in individuals with or without (liability for) psychotic disorder. 
In a cross-sectional analysis of the baseline DTI scans of the current sample, patientspecific microstructural WM alterations were found ${ }^{2}$. These alterations remained relatively stable over a 3-year time course in contrast to a decline in mean FA in the non-affected siblings compared to healthy controls ${ }^{26}$. Consequently, both patients and siblings had decreased FA with respect to controls at follow-up. The aim of the current investigation was to study whether microstructural alterations over time were conditional on the exposure to two environmental risk factors for schizophrenia. More specifically, we hypothesized that individuals at increased genetic risk (patients and siblings) with higher levels of exposure to cannabis and childhood trauma would show reduced WM FA over time.

\section{Methods}

\section{Participants}

Subjects were recruited in the context of a multicentre longitudinal study (Genetic Risk and Outcome of Psychosis, G.R.O.U.P.) in the Netherlands ${ }^{27}$. At baseline, 300 participants were included of which 258 underwent a DTI scan. At follow-up, approximately three years later (mean: 3.3 years), DTI scans were acquired from a sample of 180 participants, of which 159 provided a valid pair of DTI scans for the longitudinal analysis (see Domen et al. $(2013,2016)$ and the supplemental method section for further information on in- and exclusion criteria, family composition and diagnostic assessments) ${ }^{2,26}$.

The standing ethics committee approved the study protocol, and all participants gave written informed consent in accordance with the committee's guidelines.

\section{Measures}

Level of psychotic symptomatology at the time of scanning was assessed with the Positive and Negative Symptom Scale (PANSS) ${ }^{28}$.

Educational level was defined as highest accomplished level of education. Handedness was assessed using the Annett Handedness Scale ${ }^{29}$.

\section{AP medication}

The determination of (lifetime) antipsychotic (AP) medication use at baseline and cumulative AP exposure during the 3-year follow-up period has been described in the Supplemental Method section based on Domen et al., $2013^{2}, 2016^{26}$. 


\section{Substance use}

Substance use was measured at both time points with the Composite International Diagnostic Interview (CIDI) sections B-J-L ${ }^{30}$. As data of drug use of the last 3 years was not available, use of cannabis and other drugs was assessed as reported frequency of use during the last 12 months, as well as lifetime use (mean number of times until baseline measurement). CIDI frequency data on alcohol (weekly consumptions), lifetime cannabis and other drug use was available at follow-up for respectively 158 participants ( $1 \%$ missing data), 155 participants (3\% missing data) and 157 participants ( $1 \%$ missing data).

\section{Developmental trauma}

Developmental trauma was assessed at baseline with the Dutch version of the Childhood Trauma Questionnaire Short Form (CTQ). The short CTQ consists of 25 items rated on a 5 -point Likert scale ( $1=$ never true to $5=$ very often true) inquiring about traumatic experiences in childhood. Five types of childhood maltreatment were assessed: emotional, physical and sexual abuse, and emotional and physical neglect, with five questions covering each type of trauma ${ }^{31}$. The mean of these 25 items (range: 5.0 - 25.0) created a general measure of developmental trauma. The CTQ data were missing for 1 patient.

\section{Image acquisition}

Magnetic resonance imaging scans were obtained at Maastricht University, the Netherlands, using an Allegra Magnetom MR (Siemens, Erlangen, Germany) operating at 3.0 Tesla. At both measurement points, microstructural anatomy was examined using diffusion tensor imaging with an echo-planar-imaging sequence (field of view $230 \times 230 \mathrm{~mm}^{2}$, TR $10800 \mathrm{~ms}$, TE $84 \mathrm{~ms}$, voxel size $1.8 \times 1.8 \times 1.8 \mathrm{~mm}^{3}$, b-value 1000 $\mathrm{s} / \mathrm{mm}^{2}, 85$ slices, no overlap). As a result of an update of the scanner software during baseline acquisition, two DTI sequences were used: one with 76 directions (of which four T2-weighted (B0) and 72 diffusion-weighted (B1000)) and one with 81 directions $(8 \times \mathrm{B} 0$ and $73 \times \mathrm{B} 1000)$. Gradient directions were identical in both sequences. A potential association between the proportion of baseline scans and group was investigated using a Pearson chi-square test.

At follow-up, the DTI sequence comprised 81 directions ( $8 \times \mathrm{B} 0$ and $73 \times \mathrm{B} 1000)$. Total acquisition time of the DTI sequence was 15 minutes.

\section{Diffusion tensor imaging analysis}

Processing of DTI data was performed using tract-based spatial statistics (TBSS) v1.2 in FSL 4.1.6 (FMRIB Analysis Group, Oxford, UK, http://www.fmrib.ox.ac.uk/analysis/ research/tbss). The consecutive processing steps until the nonlinear transformation 
of the FA volumes into standard space are described in the Supplemental Method section (based on Domen et al., 2013 ${ }^{2}, 2016^{26}$ ). For the current study, three mean FA skeletons were created; for the cross-sectional analysis at baseline ( $n=258$ : controls, siblings, patients) and at follow-up $(n=180)$ and, for the longitudinal analysis, one based on six groups ( 3 groups $\times 2$ time-points). The mean FA skeleton follows the major WM tracts in each individual participant (normalized in MNI152 space) and provides a way to compare between (groups of) participants. The FA threshold was set, using visual inspection of the FA skeleton, at a level of 0.25 , to include major WM tracts whilst removing small peripheral tracts that would cause excess inter-participant variability. In addition, this threshold setting avoided inclusion of regions that are likely to be composed of multiple tissue types or fibre orientations. In the final step, a binary skeleton mask of the individual participants was created and used to extract FA values of most large WM tracts $(n=38)$ in order to create a whole-brain mean FA for the subsequent statistical analyses. The Johns Hopkins University International Consortium for Brain Mapping (JHU ICBM)-DTI-81 white-matter atlas labels ${ }^{33}$ and the JHU white-matter tractography atlas ${ }^{34}$ were used to identify and assign a specific tract name to as much voxels as possible.

\section{Statistical analyses}

In order to use a multilevel (mixed-effects) model and to be able to calculate a mean FA "change" (mean FA at baseline minus mean FA at follow-up) for the longitudinal model, which was not compatible with the standard protocol in TBSS, data were analysed in $\mathrm{R}$ (version 3.2.0), a free software environment for statistical computing and graphics ${ }^{35}$. From the 38 labelled WM tracts, skeleton mean FA values per participant per time point were extracted and exported to $R$. Since the mean FA values per subject were based on varying number of voxels, depending on the region, we used a model in which the error variance for a particular observation was inversely weighted by the number of voxels within the corresponding region.

\section{Cross-sectional analysis at baseline and follow-up}

To examine cross-sectional associations between the environmental exposures (lifetime and last year cannabis use, childhood trauma) and whole-brain mean FA at baseline $(n=258)$ and at follow-up $(n=180)$, the data set was transformed from a wide to a long format, resulting in a hierarchically structured data set, based on 38 regional mean FA measures (Level 1) nested in subjects (Level 2) who were part of the same families (Level 3). Because of the three-level grouping structure of the data, compromising statistical independence of the observations, a multilevel (mixed-effects) model was fitted. FA was the dependent variable, group (dummy variables with the controls as the reference category, controls $=0$, siblings $=1$, patients $=2$,) and the 
environmental exposures (lifetime and last year cannabis exposure, trauma exposure) were the independent variables and random effects (intercepts) were added for both subject and family.

The statistical basic model was: $F A=\beta_{0}+\beta_{1}$ (group) $+\beta_{2}$ (environmental exposure) + $\beta_{3}$ (group $\times$ environmental exposure). This model included the a priori hypothesized confounding variables age, sex, and level of education as fixed effects. In case of significant findings, additional confounding variables: Body Mass Index (BMI), alcohol consumption, and lifetime other drug use were separately added.

Main effects of the environmental exposures (controlled for group), as well as group $x$ environmental exposures interactions in the model of FA were examined. The environmental exposures were entered both as linear and as factored variables (i.e. representing the distribution of scores divided by its tertiles: lifetime cannabis use: no, moderate or heavy cannabis use; childhood trauma exposure: low, moderate, or high trauma exposure), allowing visualization of dose-response. In case of significant interaction effects, stratified analyses were conducted in order to quantify whether the association between environmental exposure and FA differed between the three groups.

To examine whether childhood trauma and lifetime cannabis use contributed independent effects, planned sensitivity analyses were performed with both environmental exposures in the model.

To examine whether the scanner software update at baseline affected the results, the interaction analyses at baseline were repeated in subgroups stratified by the number of scan directions: 76 ( $n=191)$ vs. $81(n=67)$ directions.

\section{Longitudinal analysis}

A mean FA "change" (delta, $\Delta$ ) per participant per region $(n=159)$ was calculated by subtracting mean FA at baseline from mean FA at follow-up. The same procedure, basic statistical model and analyses were applied as described in the cross-sectional part, now using $\triangle \mathrm{FA}$ as the dependent variable and group and the environmental exposures (lifetime and last year cannabis exposure, trauma exposure) as the independent variables.

In case of a significant group effect for whole-brain mean $\triangle F A$, a region-of-interest (ROI) analysis was performed, examining group $\times$ environmental exposure (linear variable) interactions in four WM tracts: left posterior thalamic radiation (PTR), right retrolenticular part of the internal capsule (RPIC), right cingulum, and right posterior corona radiate (PCR). These WM tracts were chosen because they showed, in the current study population, a significant group difference in $\triangle F A^{26}$, and they have been associated with cannabis use and childhood trauma in human and animal studies 14, 36-39. In addition, to control for a potential effect of depression on $\Delta F A$, the main 
longitudinal analysis was repeated with exclusion of the subgroup of participants in the control $(n=11)$ and sibling group $(n=15)$ with a history of a depressive disorder. Since our previous study showed a small effect of last 3-year and lifetime antipsychotic (AP) use on $\triangle F A^{26}$, planned sensitivity analyses were performed to rule out potential AP effects, using patient subgroups (with low, moderate and high AP exposure (for the number of participants per subgroup see Supplemental Table)). Thus, the group $x$ environmental exposure interactions in the model of $\triangle F A$ were examined in three AP subgroup to ascertain whether a potential effect remained significant in the respective subgroups.

\section{Results}

\section{Demographics}

The patients represented a relatively stable population (not in need of inpatient care or intensive treatment), as reflected by the low PANSS scores and the number of patients that fulfilled the remission criteria (Table 1). The gender distribution in the samples was skewed, showing more male patients and male siblings as high cannabis users and more male patients and female controls exposed to high levels of childhood trauma at follow-up (Table 2). The mean current dosage of AP medication in terms of standard haloperidol equivalents was 5.5 milligrams $(\mathrm{mg})(\mathrm{SD}=4.6)$ at baseline and $4.7 \mathrm{mg}$ ( $S D=5.1$ ) at follow-up (over the last 3 years). The proportion of baseline scans with 76 directions did not differ between the groups ( $84 \%$ in controls, $82 \%$ in siblings, and $71 \%$ in patients: $\chi^{2}=3.02, d f=2, P=.22$ ). 


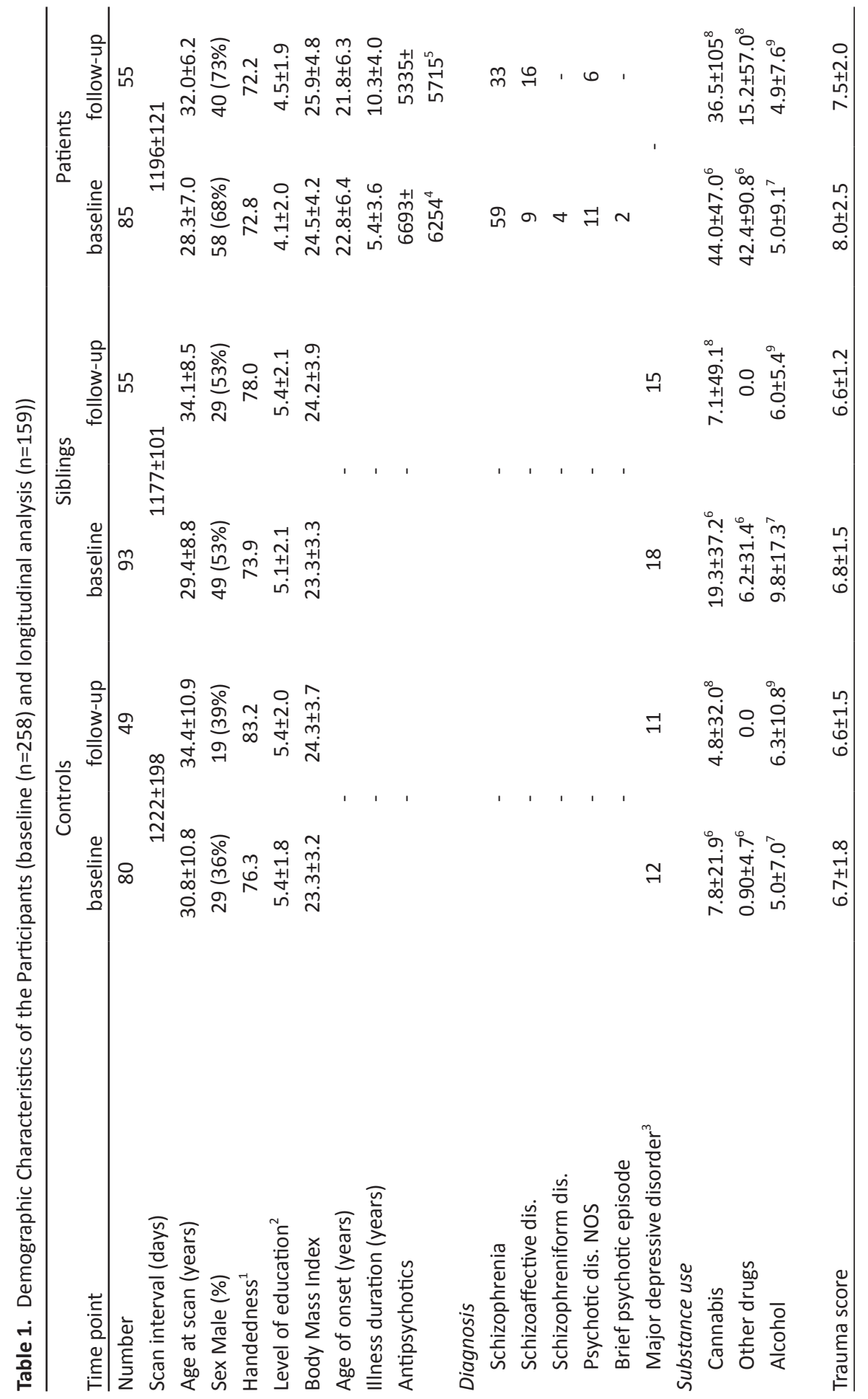




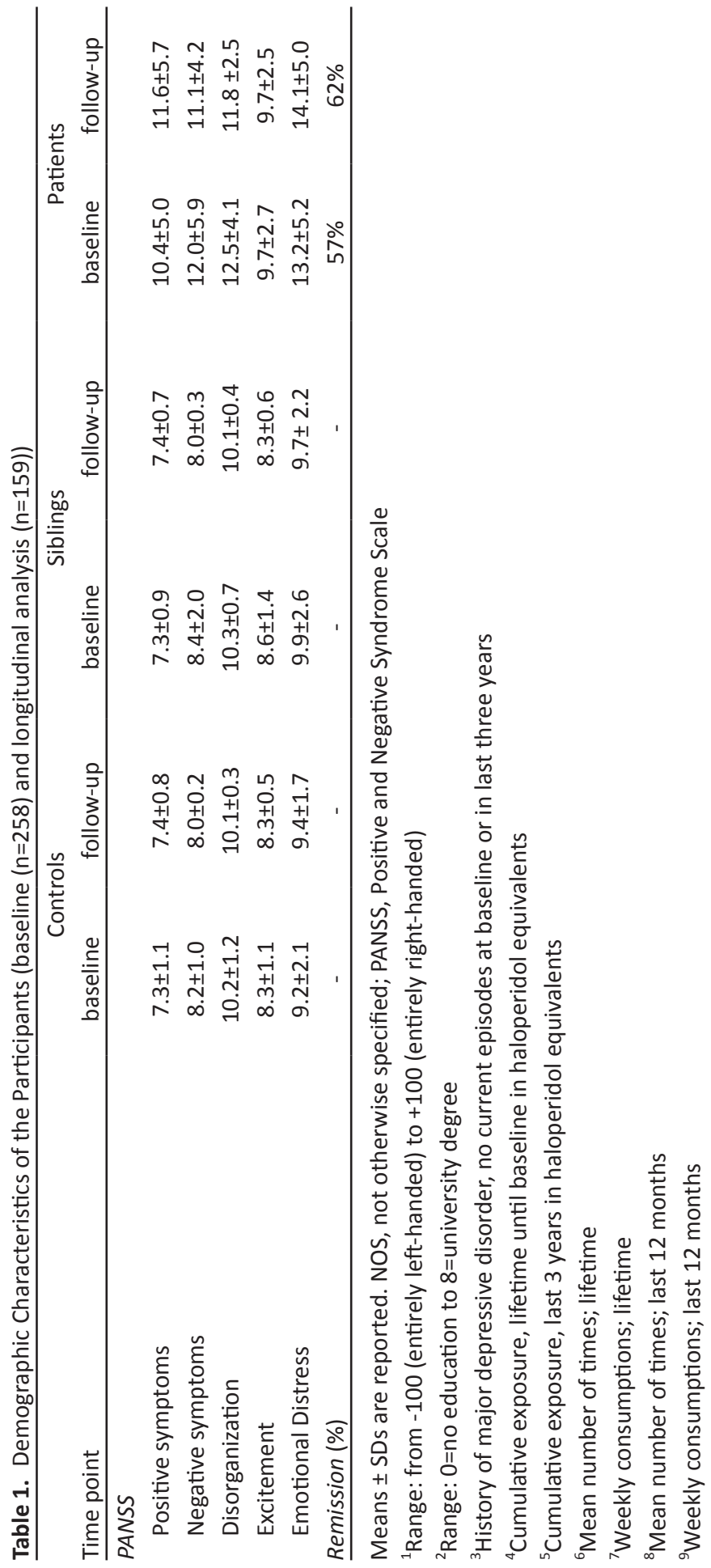


Table 2. Within-group distribution of the environmental exposures

\begin{tabular}{lcccccc}
\hline & \multicolumn{2}{c}{ Cannabis use } & \multicolumn{4}{c}{ Childhood trauma } \\
& baseline & follow-up & $\mathrm{m} / \mathrm{f}$ ratio & baseline & follow-up & $\mathrm{m} / \mathrm{f}$ ratio \\
\hline Controls & & & & & & \\
no (low) & $2204(73 \%)$ & $1406(76 \%)$ & $38 / 62$ & $1444(48 \%)$ & $798(43 \%)$ & $52 / 48$ \\
medium & $304(10 \%)$ & $190(10 \%)$ & $40 / 60$ & $988(32 \%)$ & $684(37 \%)$ & $28 / 72$ \\
high & $494(17 \%)$ & $266(14 \%)$ & $43 / 57$ & $608(20 \%)$ & $380(20 \%)$ & $30 / 70$ \\
Siblings & & & & & & \\
no (low) & $2242(64 \%)$ & $1330(64 \%)$ & $49 / 51$ & $1368(39 \%)$ & $874(42 \%)$ & $52 / 48$ \\
medium & $418(12 \%)$ & $228(11 \%)$ & $33 / 67$ & $1292(36 \%)$ & $684(33 \%)$ & $59 / 41$ \\
high & $836(24 \%)$ & $532(25 \%)$ & $71 / 29$ & $874(25 \%)$ & $532(25 \%)$ & $43 / 57$ \\
Patients & & & & & & \\
no (low) & $1178(39 \%)$ & $760(39 \%)$ & $60 / 40$ & $798(25 \%)$ & $646(31 \%)$ & $53 / 47$ \\
medium & $228(8 \%)$ & $190(10 \%)$ & $20 / 80$ & $836(26 \%)$ & $570(27 \%)$ & $80 / 20$ \\
high & $1634(53 \%)$ & $988(51 \%)$ & $88 / 12$ & $1558(49 \%)$ & $874(42 \%)$ & $83 / 17$ \\
\hline
\end{tabular}

The number of observations (=number of subjects $\times$ number of regions (38)) per group (and proportion of total group) for the baseline $(n=258)$ and longitudinal analysis $(n=159)$ per environmental stress factor

$\mathrm{m} / \mathrm{f}$ ratio, male/female ratio at follow-up

\section{Cross-sectional analysis of FA and environmental risk factors at baseline}

There were no significant associations between cannabis exposure and FA (lifetime: $B=0.002, P=0.24$, last year: $B=1.0 \times 10^{-5}, P=0.43$ ) or between trauma exposure and $F A$ $(B=-0.001, P=0.60)$. In addition, no significant interactions were found between cannabis exposure and group in the model of FA (lifetime: $\chi^{2}=1.3, d f=2, p=0.52$, last year: $\left.\chi^{2}=0.1, d f=2, p=0.93\right)$ and between trauma and group in the model of $F A\left(\chi^{2}=2.9, d f=2\right.$, $p=0.24$ ) (all analyses with linear environmental variables). The results did not change after stratification by number of scan directions (76 directions: cannabis exposure; lifetime: $\chi^{2}=1.4, d f=2, p=0.49$, last year: $\chi^{2}=0.2, d f=2, p=0.89$, trauma exposure: $\chi^{2}=2.3$, $\mathrm{df}=2, p=0.31$; and 81 directions: cannabis exposure; lifetime: $\chi^{2}=1.2, d f=2, p=0.56$, last year: $\chi^{2}=1.5, d f=2, p=0.48$, trauma exposure: $\chi^{2}=0.2, d f=2, p=0.93$ ).

\section{Cross-sectional analysis of FA and environmental risk factors at follow-up}

No significant associations between cannabis exposure (linear variable) and FA (lifetime: $\mathrm{B}=1.0 \times 10^{-4}, \mathrm{P}=0.95$, last year: $\mathrm{B}=-3.0 \times 10^{-6}, \mathrm{P}=0.88$ ) or between trauma exposure (linear variable) and $F A(B=-0.001, P=0.67)$ were found at follow-up.

\section{Cannabis}

No significant interaction was found between last year cannabis use and group in the model of $F A\left(\chi^{2}=0.8, d f=2, p=0.66\right)$. A significant interaction was found between 
respectively (lifetime) cannabis exposure $\left(\chi^{2}=9.3, d f=2, p=0.01\right)$ and group in the model of FA. This interaction remained significant after controlling for childhood trauma $\left(\chi^{2}=9.6, d f=2, p=0.008\right), B M I\left(\chi^{2}=9.7, d f=2, p=0.008\right)$, alcohol use $\left(\chi^{2}=9.8, d f=2\right.$, $p=0.008)$, and other drug use $\left(\chi^{2}=7.6, d f=2, p=0.02\right)$ (all linear variable).

Stratified analyses showed a mean FA decrease in the heavy cannabis using patients, which was significantly different from the controls and the siblings. An association in opposite direction was found in cannabis-using siblings, but only for the moderate cannabis exposure level and not for the highest exposure level (See Table 3).

\section{Trauma}

A significant interaction between trauma exposure and group in the model of FA $\left(\chi^{2}=6.1, d f=2, p=0.05\right)$ was found. The interaction remained significant after controlling for alcohol use $\left(\chi^{2}=8.8, d f=2, p=0.01\right)$, trend-significant after controlling for lifetime cannabis use $\left(\chi^{2}=4.9, d f=2, p=0.09\right)$ and $B M I\left(\chi^{2}=5.5, d f=2, p=0.06\right)$, though was not significant after addition of other drug use $\left(\chi^{2}=4.4, d f=2, p=0.11\right)$ (all linear variable). Stratified analyses showed a mean FA decrease in patients exposed to a high childhood trauma level, which was significantly different from the siblings and trend- significantly different from the controls (See Table 3).

\section{Longitudinal analysis of $\triangle \mathrm{FA}$ and environmental risk factors}

In the whole group, there was no significant association between respectively lifetime cannabis exposure $\left(B=-3.0 \times 10^{-5}, P=0.16\right)$, last year cannabis exposure $\left(B=-1.0 \times 10^{-5}\right.$, $\mathrm{P}=0.45$ ) or trauma exposure (all linear variable) and $\Delta F A\left(B=-7.0 \times 10^{-4}, P=0.13\right)$.

\section{Cannabis}

There was a significant interaction between (lifetime) cannabis exposure and group in the model of $\triangle F A\left(\chi^{2}=6.2, d f=2, p=0.04\right)$. This interaction remained significant after controlling for childhood trauma $\left(\chi^{2}=5.9, d f=2, p=0.05\right), B M I\left(\chi^{2}=5.8, d f=2, p=0.05\right)$, alcohol use $\left(\chi^{2}=5.9, d f=2, p=0.05\right)$, other drug use $\left(\chi^{2}=7.4, d f=2, p=0.02\right)$, and scan type $\left(\chi^{2}=6.5, d f=2, p=0.04\right)$. After exclusion of the 26 participants with a history of depression ( $16 \%$ reduction in sample size), the interaction became trend-significant $\left(\chi^{2}=5.4, d f=2, p=0.07\right)$ (all linear variable).

Stratified analyses showed a significant effect in patients, indicating a decrease in FA over time with increasing cannabis exposure in patients, resulting in a significant group difference between patients and controls and between patients and siblings. Compared to patients with no cannabis use, patients with heavy cannabis consumption had significantly more FA decrease over time. This was not the case for the moderate versus no cannabis use comparison (Table 4, Fig. 1). 


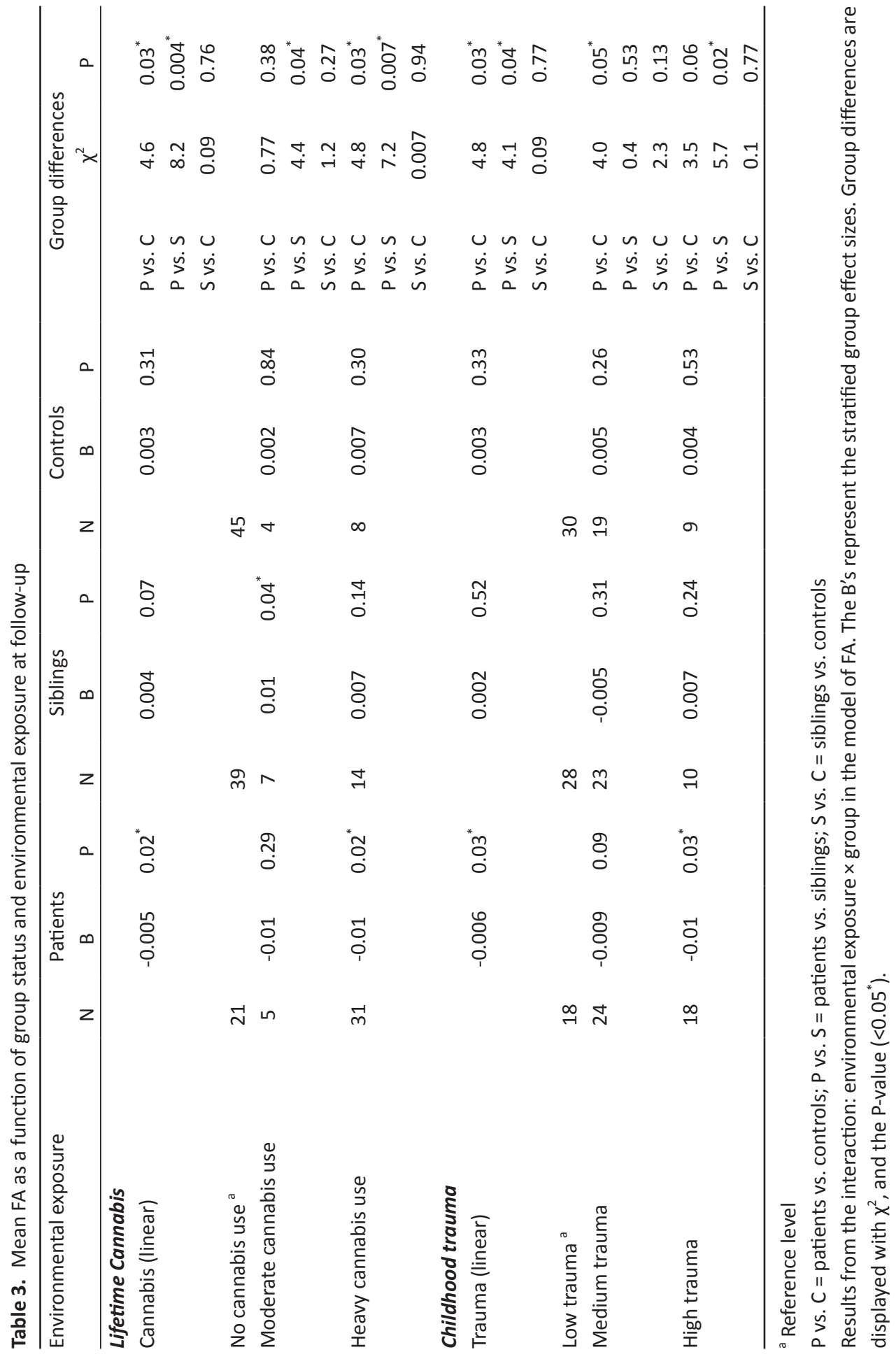




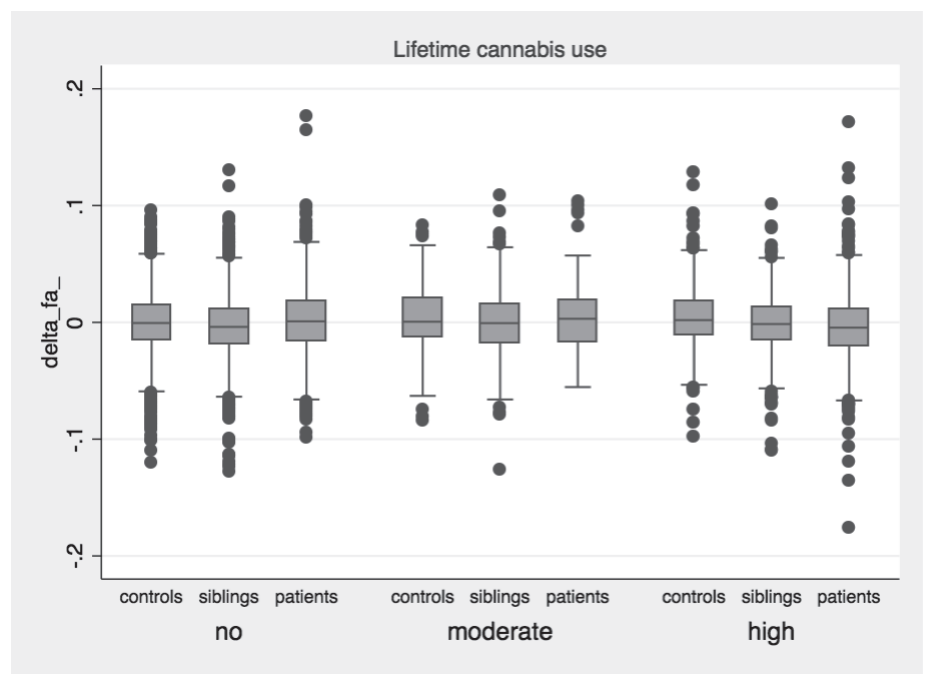

A

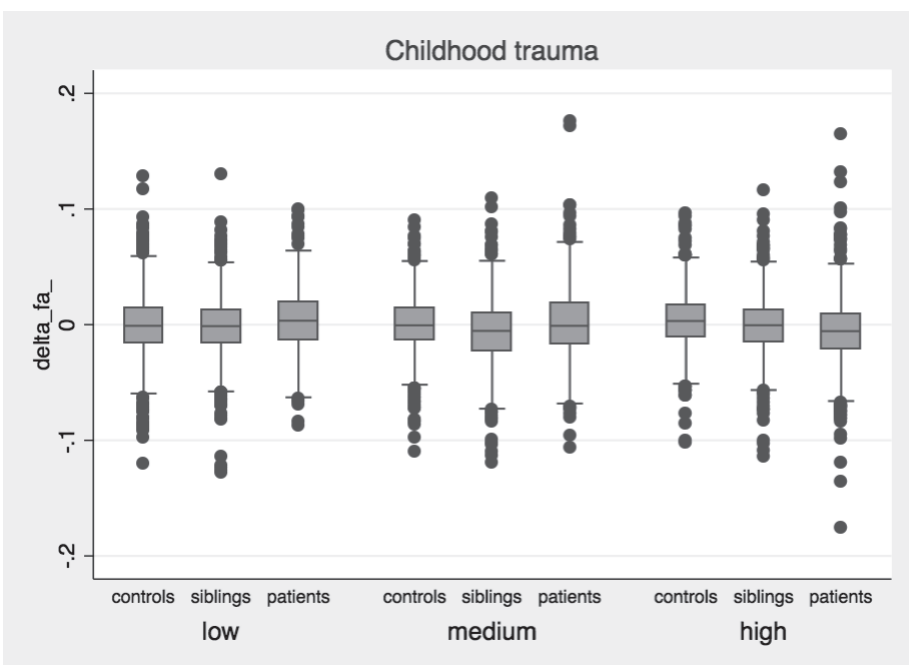

Fig. 1. The association between respectively cannabis (A) and childhood trauma (B) (dummy variables) and $\triangle \mathrm{FA}$, stratified per group. The effect of high cannabis exposure vs. no cannabis exposure and high trauma exposure vs. low trauma exposure on mean whole brain $\Delta \mathrm{F}$ was significantly different for patients compared to controls (cannabis; $\chi^{2}=3.7, p=0.05$, trauma; $\chi^{2}=10.0, p=0.002$ ) and for patients compared to siblings (cannabis; $\chi^{2}=4.3, p=0.04$, trauma; $\chi^{2}=7.2$, $p=0.007)\left({ }^{*} p<0.05\right)$. 
Table 4. Mean $\triangle F A$ as a function of group status and environmental exposure

\begin{tabular}{|c|c|c|c|c|c|c|}
\hline \multirow[t]{2}{*}{ Environmental exposure } & \multicolumn{3}{|c|}{ Patients } & \multicolumn{3}{|c|}{ Siblings } \\
\hline & $\mathrm{N}$ & $\Delta \mathrm{FA}$ & B & $P$ & $\mathrm{~N}$ & $\Delta \mathrm{FA}$ \\
\hline \multicolumn{7}{|l|}{ Lifetime Cannabis } \\
\hline Cannabis (linear) & & & -0.003 & $0.02^{*}$ & & \\
\hline No cannabis use ${ }^{a}$ & 20 & $0.0014 \pm 0.03$ & & & 35 & $-0.0040 \pm 0.03$ \\
\hline Moderate cannabis use & 5 & $0.0038 \pm 0.03$ & 0.002 & 0.60 & 6 & $0.0003 \pm 0.03$ \\
\hline
\end{tabular}

Heavy cannabis use

$26-0.0041 \pm 0.03 \quad-0.00$

$0.02^{*} \quad 14 \quad-0.0022 \pm 0.03$

\section{Childhood trauma}

Trauma (linear)

$-0.0040 .0005^{*}$

Low trauma ${ }^{\text {a }}$
Medium trauma

High trauma
$17 \quad 0.0034 \pm 0.03$

$15 \quad 0.0016 \pm 0.03$

$-0.002$

0.42

23

18

$23-0.0060 \pm 0.03$
$-0.009$

$0.0005^{*} 14$
$-0.0004 \pm 0.03$

\footnotetext{
${ }^{a}$ Reference level

$P$ vs. $C=$ patients vs. controls; $P$ vs. $S=$ patients vs. siblings; $S$ vs. $C=$ siblings vs. controls, $\triangle \mathrm{FA}$ : means and $\mathrm{SD}$ are reported.

Results from the interaction: environmental exposure $\times$ group in the model of $\triangle F A$. The $B^{\prime} s$ represent the stratified group effect sizes. Group differences are displayed with $\chi^{2}$, and the P-value $\left(<0.05^{*}\right)$.
} 


\begin{tabular}{|c|c|c|c|c|c|c|c|c|}
\hline \multicolumn{3}{|c|}{ Siblings } & \multicolumn{3}{|c|}{ Controls } & \multicolumn{3}{|c|}{ Group differences } \\
\hline B & $\mathrm{P}$ & $\mathrm{N}$ & $\Delta \mathrm{FA}$ & B & $\mathrm{P}$ & & $\chi^{2}$ & $P$ \\
\hline \multirow[t]{4}{*}{0.001} & 0.41 & & & 0.001 & 0.39 & P vs. C & 4.1 & $0.04^{*}$ \\
\hline & & & & & & P vs. S & 4.7 & $0.03^{*}$ \\
\hline & & & & & & S vs. C & 0.03 & 0.87 \\
\hline & & 37 & $-0.0005 \pm 0.03$ & & & & & \\
\hline \multirow[t]{3}{*}{0.004} & 0.25 & 5 & $0.0040 \pm 0.03$ & 0.003 & 0.47 & P vs. C & 0.02 & 0.90 \\
\hline & & & & & & P vs. S & 0.1 & 0.71 \\
\hline & & & & & & S vs. C & 0.1 & 0.80 \\
\hline \multirow[t]{3}{*}{0.002} & 0.51 & 7 & $0.0040 \pm 0.03$ & 0.002 & 0.47 & P vs. C & 3.7 & $0.05^{*}$ \\
\hline & & & & & & P vs. S & 4.3 & $0.04^{*}$ \\
\hline & & & & & & S vs. C & 0.03 & 0.86 \\
\hline \multirow[t]{4}{*}{0.0002} & 0.90 & & & 0.002 & 0.26 & P vs. C & 9.7 & $0.002^{*}$ \\
\hline & & & & & & P vs. S & 6.4 & $0.01^{*}$ \\
\hline & & & & & & S vs. C & 0.6 & 0.45 \\
\hline & & 21 & $-0.00003 \pm 0.03$ & & & & & \\
\hline \multirow[t]{3}{*}{-0.003} & 0.17 & 18 & $-0.00002 \pm 0.03$ & 0.0008 & 0.75 & P vs. C & 0.7 & 0.42 \\
\hline & & & & & & P vs. S & 0.1 & 0.76 \\
\hline & & & & & & S vs. C & 1.4 & 0.24 \\
\hline \multirow[t]{3}{*}{0.001} & 0.71 & 10 & $0.0031 \pm 0.03$ & 0.004 & 0.23 & P vs. C & 10.0 & $0.002^{*}$ \\
\hline & & & & & & Pvs. S & 7.2 & $0.007^{*}$ \\
\hline & & & & & & S vs. C & 0.4 & 0.50 \\
\hline
\end{tabular}


In the ROI analyses, no significant interactions were found between cannabis exposure and group in the model of $\triangle F A$, except for the right cingulum, but the stratified analyses did not reach significance (Table 5).

Table 5. Group $\times$ environmental exposure in the model of mean $\triangle F A$ in four WM tracts

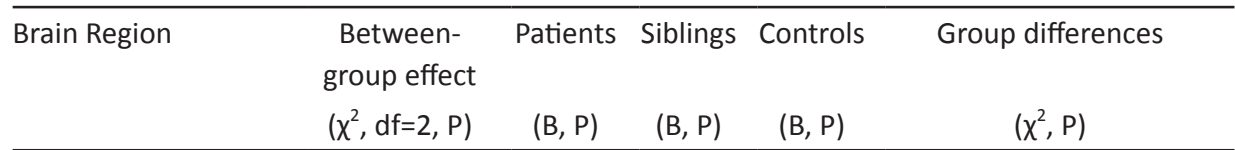

\section{LT Cannabis use}

RPIC, right $1.3,0.52$

PCR, right

Cingulum, right

PTR, left

$1.5,0.48$

\section{Childhood trauma}

RPIC, right

$10.3,0.006^{*}$

$\begin{array}{ccc}-0.01, & 0.01, & -0.002 \\ 0.02^{*} & 0.04^{*} & 0.71\end{array}$

$2.5,0.28$

$\mathrm{PCR}$, right

Cingulum, right

$\begin{array}{ccc}-0.01, & 0.01, & 0.01 \\ 0.10 & 0.19 & 0.19\end{array}$

$\begin{array}{lll}0.02-0.04 & 0.71\end{array}$
P vs. C: $1.4,0.23$

P vs. S: $10.3,0.001^{*}$

$S$ vs. C: $2.4,0.12$

P vs. C: $3.6,0.06$

P vs. S: $10.3,0.001^{*}$

S vs. C: $1.1,0.30$

PTR, left

$3.7,0.15$

Results from multilevel modelling: the $\chi^{2}$ and the P-values represent the significant results of the group $\times$ environmental exposure in model of $\triangle F A$ per WM tract ( $\left.{ }^{*} \mathrm{P}<0.05\right)$. Group differences per WM tract are shown $\left(\chi^{2}, P\right.$-value, $\left.{ }^{*} P<0.05\right)$. Analyses are controlled for age, sex, level of education, and scan interval.

$\mathrm{LT}=$ Lifetime, $\mathrm{P}$ vs. $\mathrm{C}=$ patients vs. controls; $\mathrm{P}$ vs. $\mathrm{S}=$ patients vs. siblings; $\mathrm{S}$ vs. $\mathrm{C}=$ siblings vs. controls, $\mathrm{RPIC}=$ Retrolenticular part of internal capsule, $\mathrm{PCR}=$ Posterior corona radiata, PTR=Posterior thalamic radiation

\section{Trauma}

A significant interaction between trauma exposure and group in the model of $\triangle F A$ $\left(\chi^{2}=11.3, d f=2, p=0.003\right)$ was found. The interaction remained significant after controlling for lifetime cannabis use $\left(\chi^{2}=12.3, d f=2, p=0.002\right), B M I\left(\chi^{2}=10.5, d f=2, p=0.005\right)$, alcohol use $\left(\chi^{2}=12.0, d f=2, p=0.003\right)$, other drug use $\left(\chi^{2}=14.6, d f=2, p=0.0007\right)$, and scan type $\left(\chi^{2}=11.5, d f=2, p=0.003\right)$. The interaction was nearly significant after exclusion of the 26 participants with a history of depression $\left(\chi^{2}=5.6, d f=2, p=0.06\right.$ ) (all linear variable). 
Stratified analysis revealed a significant negative association between trauma and $\triangle F A$ in patients, resulting in a significant patient - control and patient - sibling difference. Compared to low trauma exposure, patients exposed to high trauma levels had significantly more FA decrease over time. This was not the case for moderate compared to low trauma exposure (Table 4, Fig. 1).

In the ROI analysis, two of the four WM tracts showed significant interactions between trauma exposure and group in the model of $\triangle \mathrm{FA}$ : the right RPIC and the right cingulum. In the right RPIC, higher trauma levels were significantly associated with more FA decrease in patients and with more FA increase in siblings. The slopes were significantly different for the patient-sibling comparison, but not for the patientcontrol and sibling-control comparison. In the right cingulum, a significant negative association between trauma and $\triangle \mathrm{FA}$ was found in patients only, resulting in a borderline significant patient-control and a significant patient-sibling difference (Table 5).

\section{Sensitivity analyses in AP medication subgroups}

The interactions between both environmental risk factors and group in the model of $\triangle \mathrm{FA}$ were partly significant in the AP medication subgroup analyses (with smaller $\mathrm{N}$ ), most prominent in patients with the lowest (lifetime \& 3-year interval) AP exposure levels (See Supplemental Table 1).

\section{Discussion}

Despite absence of cross-sectional associations between environmental risk factors and WM fractional anisotropy at baseline, there were significant interactions between group and cannabis and trauma exposure in models of FA at follow-up and FA change over a 3-year time period. Patients exposed to the highest levels of cannabis or childhood trauma had a greater FA decrease over time compared to controls and siblings. ROI analysis revealed that the patient-related effect of childhood trauma exposure was especially apparent in the right cingulum.

\section{Cannabis exposure and white matter alterations}

Higher levels of (lifetime) cannabis exposure in patients with psychotic disorder were not associated with FA alterations at baseline, but with significant FA reduction over a 3 -year period compared to siblings and healthy controls. This is the first longitudinal study examining the effect of cannabis on WM FA in relation to (familial risk for) psychotic disorder, suggesting that the extent of WM alterations is conditional on the level of cannabis exposure in patients with the disorder. Especially, cannabis use before or at disease-onset may cause an additional reduction in WM FA, given the 
absence of an interaction between group and last year cannabis use in the model of $\triangle F A$. This finding complies with other structural imaging findings of more severe WM deficits in young adults exposed to cannabis prior to the age of $16^{40}$. It strengthens the evidence, from both preclinical human and animal models, for a neurotoxic effect of cannabis in adolescence ${ }^{41}$; a sensitive age-period for the neuronal maturation of the endocannabinoid system, possibly resulting in disrupted network connectivity of various brain areas.

To date, three longitudinal DTI studies examined substance abuse in non-psychotic populations. Reduced longitudinal FA increase was found in adolescent cannabis users $(n=23)$ in 5 clusters of fronto-parietal association fibers over a 2-year interval ${ }^{42}$, as well as a significant FA decrease over time in the left ILF in adolescents with cannabis use disorder $(n=19)$, associated with more cannabis exposure, compared to healthy controls $^{43}$. After an 18-month follow-up, poorer WM integrity was reported, although mainly predicted by alcohol, not marijuana in 7 fronto-parietal tracts in adolescent substance users $(\mathrm{n}=41)^{36}$.

In previous cross-sectional studies, positive as well as negative associations between FA and cannabis use in patients with schizophrenia have been reported. James and colleagues (2011) revealed associations between early cannabis use and decreased FA in e.g. the internal capsule, corona radiata, superior and ILF, in patients with adolescent-onset schizophrenia ${ }^{14}$. In contrast, Peters and colleagues (2009) found FA increases in the bilateral uncinate fasciculus, anterior internal capsule, and frontal WM in patients with recent onset schizophrenia who had started using cannabis before the age of 17 years, compared to a similar group with no history of cannabis use ${ }^{44}$. Reduced FA has also been found in the splenium of the corpus callosum in noncannabis using patients with schizophrenia compared to patients with schizophrenia and early onset cannabis use (before age 15 years) ${ }^{45}$. It has been shown that cannabis use may have different effects at various neurodevelopmental stages of life ${ }^{46}$. In the present study, absence of a significant cross-sectional association between FA and lifetime cannabis exposure at baseline in contrast to significant associations at followup between respectively high and moderate cannabis exposure and FA in patients and siblings compared to controls may imply an age-related effect. The effect of cannabis on brain WM may only be detectable after many years, depending on the individual WM curvatures and the timing of the measurement.

The opposite direction of effect in siblings, i.e. the FA increase with higher lifetime cannabis exposure, may suggest a delayed maturation, an imaging artifact or even a protective effect of a small amount of cannabis. A cannabinoid neuroprotective effect on brain matter with improvement in WM efficiency ${ }^{47}$ has been proposed by recent in vitro studies ${ }^{48}$. 
The negative association between especially the heavy cannabis using patients with psychotic disorder and FA may fit with the hypothesis that heavy cannabis use at a young age may alter the normal trajectory of WM brain maturation. It may thus be hypothesized that interference with the extensive pruning and myelination processes in a vulnerable adolescent brain ${ }^{49}$ and the interaction with illness-related factors may cause additional reduction in WM FA later in life. Whether this has clinical implications or influences long-term prognosis needs further investigation.

\section{Childhood trauma exposure and white matter alterations}

The present study examined the association between childhood trauma and FA in individuals with (risk for) psychotic disorder. At baseline, neither a significant interaction between trauma exposure and group in the model of FA was found, nor a main effect of trauma in any of the groups. However, at follow-up, a significant (doseresponse) negative association between trauma exposure and group in the model of FA was found in patients with psychotic disorder. In other words, higher exposure to childhood trauma is associated with lower whole-brain mean FA later in life. As with cannabis, differences in baseline and follow-up results may be explained by the timing of genetic and environmental influences (and their interactions) impacting cerebral plasticity during the life span.

The follow-up findings are in line with several studies that show reduced FA in nonpsychotic traumatized subjects ${ }^{22,50}$ in stress-processing related areas, such as the corpus callosum ${ }^{51,52}$ and the cingulum bundle ${ }^{39,53}$. Thus, cross-sectional studies indicate that the FA reductions in traumatized populations may show overlap with the WM abnormalities in schizophrenia ${ }^{1,54}$, suggesting that (part of) the WM tract alterations may be non-specific, contributing to different phenotypes.

The current study did also find a greater FA decline over a 3-year period in the patients with the highest level of childhood trauma exposure with respect to siblings and healthy controls. This significant FA decline over time associated with higher levels of childhood trauma may fit with the literature describing the neurotoxic impact of childhood trauma on WM development at a sensitive age-period ${ }^{55}$. The present finding of FA decline over time in the right cingulum, which was associated with high childhood trauma exposure, is of interest as this tract is considered part of the stress-processing neurocircuitry, connecting key structures in the limbic system as the amygdala, hypothalamus, hippocampus and frontal structures (e.g. the anterior cingulate cortex) to the rest of the brain ${ }^{56}$. It is also in line with a recent small study showing a negative association between adverse childhood experiences and FA (e.g. in the left cingulum) in patients with a diagnosis of schizophrenia, but this study did not include a control group ${ }^{57}$. 
The present study also found a patient-sibling FA difference in the RPIC, but there was no difference with respect to controls. This WM tract has not previously been related to trauma, although increased mean diffusivity has been described in individuals at clinical high risk for psychosis ${ }^{58}$ and in a subset of patients with schizophrenia experiencing negative symptoms ${ }^{59}$. The finding of a significant FA increase in siblings in this tract may be "chance" given the small subgroup of siblings with a history of childhood trauma, or it may be a biological marker of their resilience to environmental stressors. The patient-specific finding with regard to trauma may refer to a complicated interplay between trauma-related factors and psychosis-related factors to account for the more pronounced WM alterations, predominantly in the group with the highest trauma exposure. Although a causal relationship between trauma and WM decreases cannot be determined from these data, experiencing severe childhood trauma may cause an additive effect on already disrupted WM development. Alternatively, illness-related factors such as disadvantageous life style and health issues (e.g. reduced physical activity, social deprivation, smoking) ${ }^{60}$ may have contributed to increased cerebral vulnerability in patients, either or not in interaction with the environmental exposures under investigation.

\section{Methodological considerations}

Apart from the strength of this study - a relatively large longitudinal imaging design with a gene-environment approach - some limitations need to be addressed.

The sample size of some longitudinal subgroup analyses was only modest or small, resulting in loss of power and increased likelihood of false-negative results. Taken together with a rather skewed gender distribution in our sample, gender specific subanalyses were not considered feasible although it is known that several WM tracts show gender-specific FA differences ${ }^{61,62}$.

Since the effect sizes in this study are small, full understanding of the biological and clinical relevance of the results is hampered. Nevertheless, one can imagine that disproportionally higher changes may arise due to stronger regional effects, either or not in combination with higher mean trauma levels. Future studies with larger sample sizes may provide more precise estimates of regional FA effect sizes associated with these environmental exposures.

Conform the various results across studies on the association between AP use and WM alterations ${ }^{24,25,63}$, and the small effect of last 3-year and lifetime AP use on $\triangle F A$ found in our previous analyses ${ }^{26}$, the current results suggest a minor confounding effect of AP use as not all the $G \times E$ interactions remained significant in different AP subgroups. However, the results of these sensitivity analyses must be viewed with caution given the sizable lack of power (patient-AP subgroups comprised of one-third of the sample). 
It is unlikely that the two DTI sequences used at baseline would have contributed to a systematic bias, as the proportions of the two sequences were almost equal between the groups. In addition, stratified analyses (by number of scan directions) and adjustment for scanning sequence did not change the results, fitting the suggestion that the variation in tensor estimation is negligible with more than 30 diffusion directions ${ }^{64}$. Extracting mean FA values from the TBSS skeleton has the disadvantage of only examining the central portion of the WM tract, but will procure that WM was indeed examined. This is in line with more recent cannabis - diffusion studies that took a distance from a voxel-based comparison approach ${ }^{46}$.

Lastly, FA is a rather non-specific diffusion measure, containing information on myelination, fibre organization and number of axons, and therefore not completely synonymous to 'WM integrity', so that the current findings must be interpreted with caution ${ }^{65}$. Nonetheless, a whole-brain, hypothesis-generating approach was chosen, as studies investigating the influence of environmental risk factors on WM alterations in patients with psychotic disorder are scarce and ambiguous. 


\section{References}

1. Ellison-Wright I, Bullmore E. Meta-analysis of diffusion tensor imaging studies in schizophrenia. Schizophrenia research Mar 2009;108(1-3):3-10.

2. Domen PA, Michielse S, Gronenschild E, Habets P, Roebroeck A, Schruers K, van Os J, Marcelis M. Microstructural white matter alterations in psychotic disorder: A family-based diffusion tensor imaging study. Schizophrenia research May 2013;146(1-3):291-300.

3. Boos HB, Mandl RC, van Haren NE, Cahn W, van Baal GC, Kahn RS, Hulshoff Pol HE. Tract-based diffusion tensor imaging in patients with schizophrenia and their non-psychotic siblings. Eur Neuropsychopharmacol Apr 2013;23(4):295-304.

4. van Os J, Kapur S. Schizophrenia. Lancet Aug 22 2009;374(9690):635-645.

5. Cornblatt BA, Lencz T, Smith CW, Correll CU, Auther AM, Nakayama E. The schizophrenia prodrome revisited: a neurodevelopmental perspective. Schizophr Bull 2003;29(4):633-651.

6. Maynard TM, Sikich L, Lieberman JA, LaMantia AS. Neural development, cell-cell signaling, and the "two-hit" hypothesis of schizophrenia. Schizophr Bull 2001;27(3):457-476.

7. Andreasen NC, Paradiso S, O' Leary DS. "Cognitive dysmetria" as an integrative theory of schizophrenia; a dysfunction in cortical - subcortical - cerebellar circuitry. Schizophr Bull 1998;24(2):203-218.

8. Friston KJ. The Disconnection hypothesis. Schizophrenia research 1998;30:115-125.

9. Voineskos AN. Genetic underpinnings of white matter 'connectivity': Heritability, risk, and heterogeneity in schizophrenia. Schizophrenia research Jan 2015;161(1):50-60.

10. Wang F, Jiang T, Sun Z, et al. Neuregulin 1 genetic variation and anterior cingulum integrity in patients with schizophrenia and healthy controls. Journal of psychiatry \& neuroscience : JPN May 2009;34(3):181-186.

11. Molina-Holgado E, Vela JM, Arevalo-Martin A, Almazan G, Molina-Holgado F, Borrell J, Guaza C. Cannabinoids promote oligodendrocyte progenitor survival: involvement of cannabinoid receptors and phosphatidylinositol-3 kinase/Akt signaling. The Journal of neuroscience : the official journal of the Society for Neuroscience Nov 15 2002;22(22):9742-9753.

12. Cahn W, Hulshoff Pol HE, Caspers E, van Haren NE, Schnack HG, Kahn RS. Cannabis and brain morphology in recent-onset schizophrenia. Schizophrenia research Apr 1 2004;67(2-3):305307.

13. Szeszko PR, Robinson DG, Sevy S, et al. Anterior cingulate grey-matter deficits and cannabis use in first-episode schizophrenia. Br J Psychiatry Mar 2007;190:230-236.

14. James A, Hough M, James S, Winmill L, Burge L, Nijhawan S, Matthews PM, Zarei M. Greater white and grey matter changes associated with early cannabis use in adolescent-onset schizophrenia (AOS). Schizophrenia research May 2011;128(1-3):91-97.

15. Peters BD, Blaas J, de Haan L. Diffusion tensor imaging in the early phase of schizophrenia: what have we learned? J Psychiatr Res Nov 2010;44(15):993-1004.

16. Baker ST, Yucel M, Fornito A, Allen NB, Lubman DI. A systematic review of diffusion weighted $\mathrm{MRI}$ studies of white matter microstructure in adolescent substance users. Neuroscience and biobehavioral reviews Sep 2013;37(8):1713-1723.

17. Zalesky A, Solowij N, Yucel M, et al. Effect of long-term cannabis use on axonal fibre connectivity. Brain : a journal of neurology Jul 2012;135(Pt 7):2245-2255.

18. Arnone D, Barrick TR, Chengappa S, Mackay CE, Clark CA, Abou-Saleh MT. Corpus callosum damage in heavy marijuana use: preliminary evidence from diffusion tensor tractography and tract-based spatial statistics. Neurolmage Jul 1 2008;41(3):1067-1074. 
19. Pagliaccio D, Luby JL, Bogdan R, et al. Stress-system genes and life stress predict cortisol levels and amygdala and hippocampal volumes in children. Neuropsychopharmacology Apr 2014;39(5):1245-1253.

20. Teicher MH, Andersen SL, Polcari A, Anderson CM, Navalta CP. Developmental neurobiology of childhood stress and trauma. The Psychiatric clinics of North America Jun 2002;25(2):397426, vii-viii.

21. Teicher MH, Dumont NL, Ito Y, Vaituzis C, Giedd JN, Andersen SL. Childhood neglect is associated with reduced corpus callosum area. Biological Psychiatry Jul 15 2004;56(2):80-85.

22. Choi J, Jeong B, Polcari A, Rohan ML, Teicher MH. Reduced fractional anisotropy in the visual limbic pathway of young adults witnessing domestic violence in childhood. Neurolmage Jan 16 2012;59(2):1071-1079.

23. Canu E, Agosta F, Filippi M. A selective review of structural connectivity abnormalities of schizophrenic patients at different stages of the disease. Schizophr Res Jan 2015;161(1):19-28.

24. Ozcelik-Eroglu E, Ertugrul A, Oguz KK, Has AC, Karahan S, Yazici MK. Effect of clozapine on white matter integrity in patients with schizophrenia: a diffusion tensor imaging study. Psychiatry research Sep 30 2014;223(3):226-235.

25. Reis Marques $\mathrm{T}$, Taylor $\mathrm{H}$, Chaddock $\mathrm{C}$, et al. White matter integrity as a predictor of response to treatment in first episode psychosis. Brain : a journal of neurology Jan 2014;137(Pt 1):172182.

26. Domen P, Peeters S, Michielse S, Gronenschild E, Viechtbauer W, Roebroeck A, van Os J, Marcelis M. Differential Time Course of Microstructural White Matter in Patients With Psychotic Disorder and Individuals at Risk: A 3-Year Follow-up Study Schizophr Bull 2017;43(1):160-170.

27. Korver N, Quee PJ, Boos HB, Simons CJ, de Haan L. Genetic Risk and Outcome of Psychosis (GROUP), a multi-site longitudinal cohort study focused on gene-environment interaction: objectives, sample characteristics, recruitment and assessment methods. Int J Methods Psychiatr Res Sep 2012;21(3):205-221.

28. Kay SR, Fiszbein A, Opler LA. The positive and negative syndrome scale (PANSS) for schizophrenia. Schizophr Bull 1987;13(2):261-276.

29. Annett M. A classification of hand preference by association analysis. British Journal of Psychology 1970;61(3):303-321.

30. WHO. Composite International Diagnostic Interview (CIDI). 1990(Geneva: World Health Organization).

31. Bernstein DP, Ahluvalia T, Pogge D, Handelsman L. Validity of the Childhood Trauma Questionnaire in an adolescent psychiatric population. J Am Acad Child Adolesc Psychiatry Mar 1997;36(3):340-348.

32. Andreasen NC, Pressler M, Nopoulos P, Miller D, Ho BC. Antipsychotic dose equivalents and dose-years: a standardized method for comparing exposure to different drugs. Biological Psychiatry Feb 1 2010;67(3):255-262.

33. Mori S, Oishi $\mathrm{K}$, Jiang $\mathrm{H}$, et al. Stereotaxic white matter atlas based on diffusion tensor imaging in an ICBM template. Neurolmage Apr 1 2008;40(2):570-582.

34. Hua K, Zhang J, Wakana S, et al. Tract probability maps in stereotaxic spaces: analyses of white matter anatomy and tract-specific quantification. Neurolmage Jan 1 2008;39(1):336-347.

35. R: A Language and Environment for Statistical Computing [computer program]. Version 3.2.0. Vienna, Austria; 2015. 
36. Bava S, Jacobus J, Thayer RE, Tapert SF. Longitudinal changes in white matter integrity among adolescent substance users. Alcoholism, clinical and experimental research Jan 2013;37 Suppl 1:E181-189.

37. Coplan JD, Abdallah CG, Tang CY, et al. The role of early life stress in development of the anterior limb of the internal capsule in nonhuman primates. Neurosci Lett Aug 16 2010;480(2):9396.

38. Sanjuan PM, Thoma R, Claus ED, Mays N, Caprihan A. Reduced white matter integrity in the cingulum and anterior corona radiata in posttraumatic stress disorder in male combat veterans: a diffusion tensor imaging study. Psychiatry Res Dec 30 2013;214(3):260-268.

39. Wang HH, Zhang ZJ, Tan QR, et al. Psychopathological, biological, and neuroimaging characterization of posttraumatic stress disorder in survivors of a severe coalmining disaster in China. J Psychiatr Res Apr 2010;44(6):385-392.

40. Cookey J, Bernier D, Tibbo PG. White matter changes in early phase schizophrenia and cannabis use: an update and systematic review of diffusion tensor imaging studies. Schizophr Res Jul 2014;156(2-3):137-142.

41. Rubino T, Parolaro D. Cannabis abuse in adolescence and the risk of psychosis: a brief review of the preclinical evidence. Progress in neuro-psychopharmacology \& biological psychiatry Jul 3 2014;52:41-44.

42. Becker MP, Collins PF, Lim KO, Muetzel RL, Luciana M. Longitudinal changes in white matter microstructure after heavy cannabis use. Dev Cogn Neurosci Dec 2015;16:23-35.

43. Epstein KA, Kumra S. White matter fractional anisotropy over two time points in early onset schizophrenia and adolescent cannabis use disorder: A naturalistic diffusion tensor imaging study. Psychiatry research Apr 30 2015;232(1):34-41.

44. Peters BD, de Haan L, Vlieger EJ, Majoie CB, den Heeten GJ, Linszen DH. Recent-onset schizophrenia and adolescent cannabis use: MRI evidence for structural hyperconnectivity? Psychopharmacol Bull 2009;42(2):75-88.

45. Dekker N, Schmitz N, Peters BD, van Amelsvoort TA, Linszen DH, de Haan L. Cannabis use and callosal white matter structure and integrity in recent-onset schizophrenia. Psychiatry Res Jan 30 2010;181(1):51-56.

46. Jakabek D, Yucel M, Lorenzetti V, Solowij N. An MRI study of white matter tract integrity in regular cannabis users: effects of cannabis use and age. Psychopharmacology (Berl) Oct 2016;233(19-20):3627-3637.

47. Westlye LT, Walhovd KB, Dale AM, et al. Life-span changes of the human brain white matter: diffusion tensor imaging (DTI) and volumetry. Cereb Cortex Sep 2010;20(9):2055-2068.

48. Sarne Y, Mechoulam R. Cannabinoids: between neuroprotection and neurotoxicity. Curr Drug Targets CNS Neurol Disord Dec 2005;4(6):677-684.

49. Lubman DI, Cheetham A, Yucel M. Cannabis and adolescent brain development. Pharmacol Ther Apr 2015;148:1-16.

50. Daniels JK, Lamke JP, Gaebler M, Walter H, Scheel M. White matter integrity and its relationship to PTSD and childhood trauma--a systematic review and meta-analysis. Depression and anxiety Mar 2013;30(3):207-216.

51. Jackowski AP, Douglas-Palumberi H, Jackowski M, Win L, Schultz RT, Staib LW, Krystal JH, Kaufman J. Corpus callosum in maltreated children with posttraumatic stress disorder: a diffusion tensor imaging study. Psychiatry Research Apr 15 2008;162(3):256-261. 
52. Paul R, Henry L, Grieve SM, et al. The relationship between early life stress and microstructural integrity of the corpus callosum in a non-clinical population. Neuropsychiatr Dis Treat Feb 2008;4(1):193-201.

53. Zhang L, Zhang Y, Li L, et al. Different white matter abnormalities between the first-episode, treatment-naive patients with posttraumatic stress disorder and generalized anxiety disorder without comorbid conditions. Journal of affective disorders Sep 2011;133(1-2):294-299.

54. Kubicki M, McCarley R, Westin CF, Park HJ, Maier S, Kikinis R, Jolesz FA, Shenton ME. A review of diffusion tensor imaging studies in schizophrenia. J Psychiatr Res Jan-Feb 2007;41(1-2):1530.

55. Heim C, Binder EB. Current research trends in early life stress and depression: review of human studies on sensitive periods, gene-environment interactions, and epigenetics. Experimental neurology Jan 2012;233(1):102-111.

56. Dedovic K, D'Aguiar C, Pruessner JC. What stress does to your brain: a review of neuroimaging studies. Canadian journal of psychiatry Revue canadienne de psychiatrie Jan 2009;54(1):6-15.

57. Poletti S, Mazza E, Bollettini I, Locatelli C, Cavallaro R, Smeraldi E, Benedetti F. Adverse childhood experiences influence white matter microstructure in patients with schizophrenia. Psychiatry Res Aug 14 2015;234:35-43.

58. von Hohenberg CC, Pasternak O, Kubicki M, et al. White matter microstructure in individuals at clinical high risk of psychosis: a whole-brain diffusion tensor imaging study. Schizophr Bull Jul 2014;40(4):895-903.

59. Arnedo J, Mamah D, Baranger DA, et al. Decomposition of brain diffusion imaging data uncovers latent schizophrenias with distinct patterns of white matter anisotropy. Neuroimage Oct 15 2015;120:43-54.

60. von Hausswolff-Juhlin Y, Bjartveit M, Lindstrom E, Jones P. Schizophrenia and physical health problems. Acta Psychiatr Scand Supp/ 2009(438):15-21.

61. Kanaan RA, Chaddock C, Allin M, Picchioni MM, Daly E, Shergill SS, McGuire PK. Gender influence on white matter microstructure: a tract-based spatial statistics analysis. PloS one 2014;9(3):e91109.

62. Menzler K, Belke M, Wehrmann E, et al. Men and women are different: diffusion tensor imaging reveals sexual dimorphism in the microstructure of the thalamus, corpus callosum and cingulum. Neurolmage Feb 14 2011;54(4):2557-2562.

63. Szeszko PR, Robinson DG, Ashtari M, et al. Clinical and neuropsychological correlates of white matter abnormalities in recent onset schizophrenia. Neuropsychopharmacology : official publication of the American College of Neuropsychopharmacology Apr 2008;33(5):976-984.

64. Jones DK. The effect of gradient sampling schemes on measures derived from diffusion tensor MRI: a Monte Carlo study. Magnetic resonance in medicine : official journal of the Society of Magnetic Resonance in Medicine / Society of Magnetic Resonance in Medicine Apr 2004;51(4):807-815.

65. O'Donnell LJ, Pasternak O. Does diffusion MRI tell us anything about the white matter? An overview of methods and pitfalls. Schizophr Res Jan 2015;161(1):133-141. 


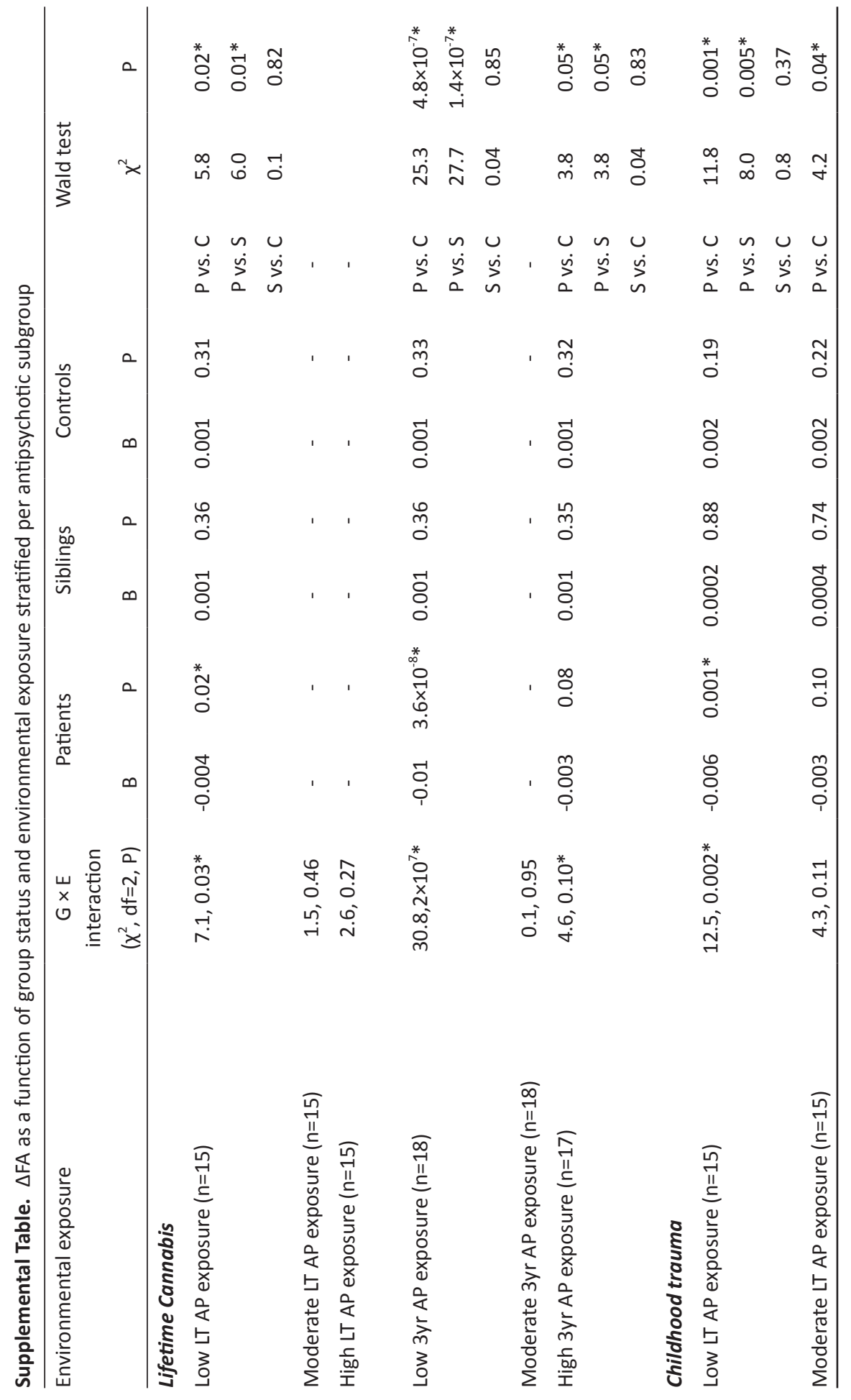




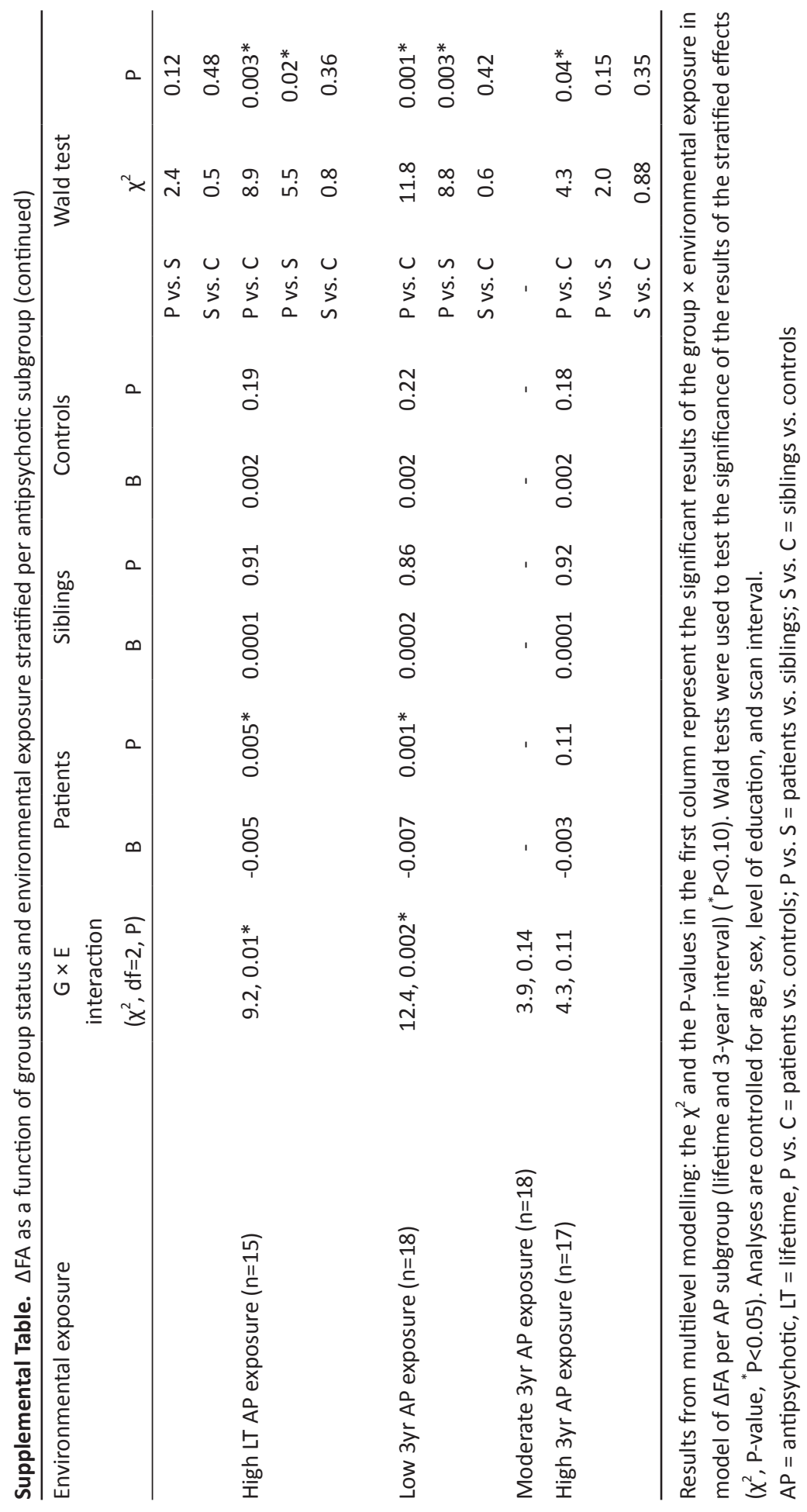



Chapter 5

Discussion 

In the present thesis, we examined microstructural white matter (WM) integrity cross-sectionally and longitudinally in patients with a psychotic disorder, unaffected siblings of patients with psychotic disorder and a healthy control group. Moreover, the role of environmental risk factors was assessed in relation to microstructural WM alterations (over time) and background psychosis-risk.

\section{Intermediate phenotype}

Investigating the etiology of a heterogeneous psychiatric syndrome as psychotic disorder is rather challenging, given the complex interaction between multiple candidate genes and an estimated modest effect of the environment ${ }^{1}$. One of the goals of this thesis was to find a measurable WM brain alteration that is present in individuals at higher than average genetic risk for psychotic disorder (unaffected siblings), as an indication for a potential intermediate phenotype ${ }^{2}$. A single intermediate phenotype associated with a single psychiatric disorder, however, is a too simplistic representation and certain intermediate phenotypes may reflect both the genetic as well as the environmental risk factor for a psychiatric disorder ${ }^{3}$. It has also been proposed that the classic endophenotype concept in the context of psychotic disorder needs renewal, given the dynamic changes displayed in the different stages of the illness. Imaging data show inconsistent results of (brain) changes prior to the onset of psychosis in clinical high-risk samples, in contrast to more solid grey and white matter findings during and after transition to psychotic disorder. Therefore, the study of early and late maturational processes via a longitudinal approach can help to identify these dynamic changes in the pre-onset stage, and subsequently may become incorporated into future endophenotype concepts ${ }^{4}$.

\section{White matter intermediate phenotype from a cross-sectional perspective}

A recent qualitative review described various alterations in WM regions (e.g. left inferior longitudinal fasciculus, anterior limb of the internal capsule, corpus callosum, cingulum) in the relatives of patients with a psychotic disorder, with FA values being intermediate compared to the values of patients and controls ${ }^{5}$. In the study presented in chapter 2, a potential WM diffusion intermediate phenotype was investigated using a whole-brain voxel-based analysis approach in a family based design. Analysis, using Tract Based Spatial Statistics (TBSS), showed widespread significantly lower mean fractional anisotropy (FA) (eg. the corpus callosum, forceps major and minor, posterior thalamic radiation bilaterally) in patients with psychotic disorder compared to unaffected siblings and healthy controls. In some regions, intermediate FA values were found in the unaffected siblings, yet siblings were not significantly different 
from controls. These results are in line with the majority of the voxel-based analysis (VBA) studies with FA as main outcome measure, showing no significant FA decreases in unaffected siblings compared to healthy controls ${ }^{6-13}$. Significant sibling - control differences were only found in two small VBA studies ${ }^{14,15}$ and five regions-of-interest (ROI) studies 7,10,11,16,17 (including three of the already mentioned negative VBA studies, in which also an ROI approach was applied ${ }^{7,10,11}$ ) (see Table 1). Taken into account all these DTI WM studies on unaffected siblings of patients with psychotic disorder, the literature is ambiguous, from which one may conclude that regional disrupted connectivity, especially in the corpus callosum, may be an endophenotype for schizophrenia ${ }^{5}$. On the other hand, the regional white matter brain pathology in the patients in combination with the absence of findings in relatives may fit the suggestion of illness and/or treatment-related alterations. A meta-analysis on this subject seems highly warranted.

\section{White matter endophenotype from a longitudinal perspective}

Given the fact that WM development shows a tract-specific, age-dependent curvilinear course, with increases into adolescence, decelerated maturation until mid-adulthood and after that a gradual decline ${ }^{18}$, genetically sensitive longitudinal diffusion studies may be best suited to discover potential structural WM endophenotypes over the course of the illness. The results of the first longitudinal family-based diffusion study were presented in chapter $\mathbf{3}$. The main findings were: (i) a significant decline in whole brain mean FA in non-psychotic siblings of patients with psychotic disorder compared to controls and (ii) a fairly stable whole-brain mean FA in patients with a psychotic disorder over a 3-year period.

There were more tracts showing a decrease than an increase in FA over time in both patients and siblings. A significant smaller FA increase in the right retrolenticular part of the internal capsule (RPIC) and a FA decrease in the right cingulum in patients and siblings compared to controls was found. Furthermore, a significant decrease in FA of the right posterior corona radiata was found in patients compared to controls and a decrease in FA of the left posterior thalamic radiation (PTR) was found in siblings with respect to patients and controls.

As the effect sizes were small and as the period of investigation only covers a relatively short period during the illness trajectory, the biological significance and clinical relevance need further investigation. Nevertheless, the findings provide information on the direction of WM FA change (increase, decrease, progression vs. static alterations) compared to a 'typical age-trajectory'. Moreover, even small decreases in microstructural WM connectivity may have a profound effect on the topological organization of the connectome, and thus could result in reduced communication between segregated brain regions ${ }^{19,20}$. 
Table 1. Summary of DTI studies comparing unaffected relatives of patients with a psychotic disorder to controls

\begin{tabular}{|c|c|c|c|}
\hline $\begin{array}{l}\text { Analysis } \\
\text { Method }\end{array}$ & Authors & $\begin{array}{c}\text { Sample } \\
\text { (Relatives-Controls) }\end{array}$ & $\begin{array}{c}\text { Results } \\
\text { (FA: Relatives-Controls) }\end{array}$ \\
\hline \multirow[t]{8}{*}{ VBA } & Domen et al. ${ }^{8}$ & $93-80$ & $\begin{array}{l}\text { No significant group differences in } \\
\text { FA }\end{array}$ \\
\hline & Boos et al. ${ }^{6}$ & $123-109$ & \\
\hline & Camchong et al. ${ }^{7}$ & $22-30$ & \\
\hline & Goghari et al. ${ }^{9}$ & $24-27$ & \\
\hline & Knochel et al. ${ }^{10}$ & $18-22$ & \\
\hline & $\begin{array}{l}\text { Munoz Maniega et } \\
\text { al. }^{11}\end{array}$ & $22-51$ & \\
\hline & Philips et al. ${ }^{12}$ & $49-21$ & \\
\hline & Prasad et al. ${ }^{13}$ & $21-29$ & \\
\hline \multirow[t]{2}{*}{ VBA } & Hao et al. ${ }^{14}$ & $34-32$ & $\begin{array}{l}\text { In relatives: } \\
\text { - FA } \downarrow \text { : left hippocampus, left } \\
\text { prefrontal cortex }\end{array}$ \\
\hline & Hoptman et al. ${ }^{15}$ & $22-37$ & $\begin{array}{l}\text { In relatives: } \\
\text { - FA } \downarrow \text { : WM of the left inferior } \\
\text { frontal gyrus, posterior cingulate } \\
\text { bilaterally, angular gyral bilaterally } \\
\text { - FA } \uparrow: \text { WM of the left subgenual } \\
\text { anterior cingulate, pontine } \\
\text { tegmental bilaterally and right } \\
\text { middle/superior frontal gyri }\end{array}$ \\
\hline \multirow[t]{5}{*}{ ROI } & Clark et al. ${ }^{16}$ & $20-32$ & $\begin{array}{l}\text { In relatives, FA } \downarrow \text { : } \\
\text { Left inferior longitudinal fasciculus }\end{array}$ \\
\hline & Knochel et al. ${ }^{17}$ & $16-15$ & $\begin{array}{l}\text { In relatives, FA } \downarrow \text { : } \\
\text { Corpus callosum }\end{array}$ \\
\hline & $\begin{array}{l}\text { Munoz Maniega et } \\
\text { al. }{ }^{11}\end{array}$ & $22-51$ & $\begin{array}{l}\text { In relatives, FA } \downarrow \text { : } \\
\text { Anterior limb of the internal capsule }\end{array}$ \\
\hline & Camchong et al. ${ }^{7}$ & $22-30$ & $\begin{array}{l}\text { In relatives, FA } \downarrow \text { : } \\
\text { Right genu of the CC }\end{array}$ \\
\hline & Knochel et al. ${ }^{10}$ & $18-22$ & $\begin{array}{l}\text { In relatives, FA } \downarrow \text { : } \\
\text { Commissural fibers, association } \\
\text { fibers and the cingulum bundle }\end{array}$ \\
\hline
\end{tabular}

VBA, Voxel-based analysis; ROI, Regions-of-interest; FA, Fractional Anisotropy; $\downarrow$, decrease; $\uparrow$, increase; WM, white matter; CC, Corpus callosum 
At follow up, a significant lower whole-brain mean FA was found in both patients and siblings compared to controls, whereas the significant difference between patients and siblings from the baseline analysis was no longer apparent. The FA difference between sibling and controls at follow-up is in line with some of the previous crosssectional sibling studies. Considering the varying mean age-ranges (20-42 years) of siblings in the different studies, this may have influenced the FA outcomes and explain some of the inconsistencies in the literature. Based on the current findings and previous cross-sectional FA studies in siblings ${ }^{5}$, the FA course over time in unaffected siblings of patients with a psychotic disorder can be described as: (i) a whole-brain mean FA similar to healthy controls and stable over time, (ii) a lower or equal mean FA compared to controls and an earlier-than-expected by age decline of FA over time, (iii) a lower mean FA compared to controls but stable over time (thus at a constantly lower level than controls).

The current results of WM alterations are indicative of an aberrant WM development curvature in siblings (whole-brain FA and regional) and patients (regional FA) and of a potential fronto-temporal (cingulum) WM intermediate phenotype. The alterations in the (right) cingulum bundle, an important white matter fibre tract of the limbic system, fit in the extensive literature on the this tract and will therefore be discussed in more detail below.

\section{Cingulum bundle}

\section{Structural alterations in the cingulum in psychotic disorder}

Due to the central role of the cingulum in emotional and cognitive processing (See Box 1), microstructural WM alterations in this particular tract have been implicated in the pathogenesis of psychotic disorder. To date, numerous ROI imaging studies have been conducted in patient samples at different stages of the disease, assessing WM alterations in the cingulate region. The evidence provided by over a dozen cross-sectional studies and one longitudinal study comparing patients with schizophrenia and healthy controls, show a consistent pattern of decreased left and right grey matter volume in the anterior cingulate gyrus ${ }^{21}$, decreased FA in especially the (right) anterior part of the cingulum ${ }^{22-25}$ and a greater decline in FA over a 4-year period in the left anterior cingulate gyrus in patients with a chronic psychotic disorder ${ }^{26}$.

As stated in a qualitative review of structural neuroimaging findings in schizophrenia by Wheeler and Voineskos (2014), less equivocal are the findings in patients with a first psychotic episode. These findings reveal a more widespread pattern of WM abnormalities, with an equal number of studies (3) showing FA decreases and absence of FA differences in the cingulum ${ }^{25}$. However, the study by Tang and colleagues (2010) 
that reported significantly reduced FA in the right anterior cingulum in 38 adolescents with early-onset schizophrenia compared to healthy volunteers ${ }^{27}$, was not included in this review.

Box 1: Role of the Cingulum

The cingulum bundle is the major WM tract, projecting from the cingulate gyrus to the entorhinal cortex, establishing the main communication pathway in the limbic system, connecting the cingulate gyrus with several other important brain regions, including the premotor, prefrontal and parietal cortices, thalamus, and hippocampus. Given the connection with the latter region, the cingulum is also associated with cognitive tasks as attention and memory. Thereby, it is part of the cingulate cortex, in particular the anterior cingulate cortex (ACC), which is primarily concerned with emotions and pain ${ }^{31}$.

It has been proposed that FA reductions in the cingulate gyrus may be associated with (subclinical) psychotic symptoms. Indeed, in patients with schizophrenia a negative association was found between FA and impulsivity in the anterior cingulate ${ }^{28}$, and between FA and PANSS positive scores in the posterior cingulum bundle (CB) bilaterally ${ }^{29}$. In addition, a trend positive association between positive symptom scores and FA in the left anterior cingulum in a small sample of recent onset patients with psychotic disorder ${ }^{30}$ has been described.

Thus, white matter alterations in the cingulate region, including symptoms-associations, have been repeatedly reported in studies on diverse populations within the psychosis continuum.

\section{Cingulum WM alteration as a potential intermediate phenotype}

Various WM abnormalities in unaffected siblings have been proposed as WM endophenotype, though not with a consistent pattern of involved neuroanatomical regions (see Table 1). The corpus callosum, however, is more frequently replicated and a potential candidate ${ }^{5}$. The data presented in this thesis show an earlier thanexpected-by age FA decline in the right cingulum in siblings. Together with evidence from two other (cross-sectional) voxel-based ${ }^{15}$ and ROI ${ }^{10}$ studies, support for the cingulum as WM endophenotype candidate is available. It is known that siblings of patients with psychotic disorder may suffer from mild subclinical positive and disorganized symptoms, reduced emotional expression, and social dysfunction ${ }^{32}$. Studies examining WM alterations in unaffected relatives nevertheless did not find significant associations between FA and subclinical positive and negative symptoms ${ }^{6}$, cognitive symptoms (sustained attention, executive functions and verbal memory ${ }^{13}$, and social and global functioning or IQ ${ }^{9}$. In contrast, a study by Knochel and colleagues (2012) 
showed a negative association in healthy relatives between whole-brain FA and the severity of positive symptoms, a predisposition towards hallucinations and subjective cognitive dysfunction ${ }^{10}$. Associations between subclinical symptoms in unaffected relatives and microstructural WM would have further strengthened the hypothesis of a white matter intermediate phenotype. However, detecting this may be complicated due to the fact that anisotropy may need to reach a critical threshold before symptom-correlations become manifest.

Overall, based on the current findings and previous literature, the cingulum seems to fit the major criteria for a reliable candidate structural endophenotype, as brain alterations in this area: (1) have been associated with genetic risk variations (e.g. SNPs in the NRGN gene ${ }^{33}$ and the ZNF804A gene $\left.{ }^{34}\right),(2)$ are present in siblings of patients with a psychotic disorder at a higher rate than the general population ${ }^{10,15},(3)$ are present without clinical psychotic symptoms. The 'ultimate' intermediate phenotype likely comprises an integrative network of functionally interacting WM pathways ${ }^{35}$. Therefore, future studies using advanced diffusion imaging techniques, combining (longitudinal) anatomical and functional measures of connectivity in large, clearly defined, samples of first-degree relatives and psychotic patients at different illness stages, should be conducted. Results of such studies are necessary to elucidate the possibility of a WM intermediate phenotype that may serve as a candidate marker for early and preclinical identification of subjects at risk or as a quantitative trait marker for future genetic studies.

\section{The role of environmental exposures in microstructural white matter alteration}

An interesting study by Chiang and colleagues (2011) showed how genetic influence on WM integrity can change over the first three decades of life in a large healthy twin-sample. WM heritability was associated with younger age (adolescence), male sex, higher IQ and higher SES, while FA was higher in adulthood. The suggestion was made that environmental influences, e.g. education, life experiences and diet, may increasingly determine brain fibre network development as one matures into adulthood ${ }^{36}$. Studies assessing environmental risks in relation to WM integrity in patients with schizophrenia are scarce, let alone in genetic high-risk populations. 


\section{$\underline{\text { Structural Imaging }}$}

In patients with psychosis vs. controls:

- Bilateral ACG: $\downarrow$ GM volume

- Anterior part Cingulum, right : $\downarrow F A$

- $\mathrm{ACG}$, left: $\downarrow \Delta \mathrm{FA}$

\section{Psychotic symptoms}

- AC: FA $<->$ Impulsivity

- Posterior CB, bilateral:

FA $<->$ PANSS positive symptoms

- $C B: \downarrow F A$ in patients with SCZ with delusions of control
In siblings vs. controls:

- Cingulum, right: $\downarrow \Delta \mathrm{FA}$

- CG, bilateral: $\downarrow F A$

- CB: $\downarrow F A$

\section{Environment}

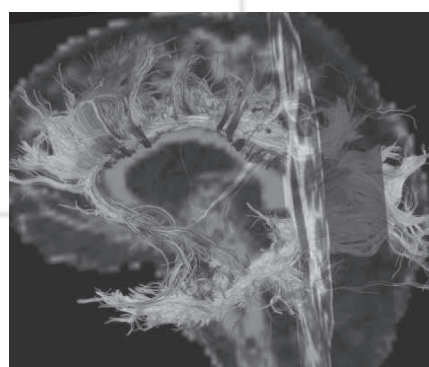

- Cingulum, right: $\triangle \mathrm{FA}<->$ Childhood trauma

- $\mathrm{CB}$, left: FA <-> Parental verbal abuse, childhood trauma

- ACG: GM volume $<->$ Cannabis use

- ACC, left: $\mathrm{CT}<->$ Cannabis use

\section{$\underline{\text { Genes }}$}

- ACC, left: $\downarrow$ GM volume in patients with SCZ with risk T allele NRGN

- $\mathrm{CG}$, left: $\downarrow F A$ in patients with $\mathrm{SCZ}$ with risk variant $r s 1344706$ of ZNF804A gene

Fig. 1. Structural imaging findings of grey and white matter alterations in the cingulum associated with psychotic symptoms, environmental exposures and genetic risk variations ACG, Anterior Cingulate Gyrus; ACC, Anterior Cingulate Cortex; AC, Anterior Cingulate; CG, Cingulate Gyrus; $C B$, Cingulum Bundle; GM, Grey Matter; FA, Fractional Anisotropy; $\Delta$, FA change over time; $\downarrow$, FA decrease; WM, white matter; $<->$, negative association; SCZ, schizophrenia; PANSS, Positive and Negative Syndrome Scale

\section{Childhood trauma}

The presented study in chapter 4 showed, for the first time, that the extent of WM alterations is conditional on the level of developmental trauma exposure in patients with a psychotic disorder. Especially patients exposed to high levels of childhood trauma showed a significant mean FA decrease compared to siblings and controls. This finding was also observed in the longitudinal design: higher levels of childhood trauma were negatively associated with white matter connectivity change during the course of the illness. This effect was not found in the siblings and controls, which may have been caused by the lower overall trauma load in these two groups in the present sample. On the other hand, maltreated siblings and controls, compared to patients, may differ in their (pre-existing or adaptive) neurobiological resilience and in their coping styles, moderating trauma effects on WM integrity. Imaging studies investigating resilience, especially in psychotic disorder, are scarce, reporting on increased fibre 
density and connectivity in respectively the anterior corpus callosum and the dorsal anterior cingulate cortex as well as on trauma associated GM volume differences in regions known for their role in the stress-circuit (see Box 2) ${ }^{37,38}$. Interestingly, in a recent review it was suggested that neurobiological differences may exist between traumatized and non-traumatized individuals, independent of the severity or even presence of psychiatric symptomatology. Thus, there may exist separate ecophenotypes with distinctive genetic, neurobiological and clinical characteristics ${ }^{37}$, stressing the importance of the integration of environmental components in biological/imaging research.

\section{Childhood trauma associated WM tract alterations}

In the current study we examined whether higher childhood trauma exposure was associated to specific (stress-related) regions. A ROI analysis was performed using the four WM tracts (left PTR, right RPIC, right cingulum, and right PCR) that had revealed significant group differences in $\triangle F A$, as described in Chapter 3.

In patients, higher childhood trauma was associated with FA decline over time in the right RPIC (which was not significantly different from controls, only from siblings) and the right cingulum (which was trend-significantly different from controls and significantly different from siblings).

Box 2: Neuro-circuitry of Stress

The amygdala plays a central role, involved in encoding and consolidation of emotional memory of events and regulating parts of the fear response by activating the hypothalamic-pituitary-adrenal (HPA) axis, which will be activated during a traumatic event. The amygdala receives auditory, visual and somatosensory input from the temporal and anterior cingulate cortices and passes output to other subcortical structures like the hypothalamus via the stria terminalis. The hippocampus, important for declarative memory, also plays a regulating role in the HPA-axis activation, with the fornix as his major output fibre bundle ${ }^{41}$. The retrolenticular part of the internal capsule, containing fibres from the optic system and the long temporo-occipital tracts in the sagittal stratum, including the inferior longitudinal fasciculus, also transferring visual signals ${ }^{42}$, may play a role in a visual limbic pathway, subserving emotional, learning and memory functions related to visual intrusions of a traumatic event.

Interestingly, the significant finding in the cingulum concurs with one of the main findings of a recent systematic review ( $n=25$ studies) on the relation of white matter integrity and childhood trauma in non-psychotic (predominantly PTSD) patient populations ${ }^{39}$. Hence, suggesting a role of the cingulate area across disorders, possibly reflecting a common pathway to trauma-related alterations, not specific to a certain 
psychiatric disorder. The two aforementioned WM tracts are both involved in the neuro-circuitry of stress, (inter) connecting key structures in the limbic system like the amygdala, hypothalamus, hippocampus and frontal structures (medial prefrontal cortex and anterior cingulate cortex) to the rest of the brain ${ }^{40}$. Previous ROI studies of non-psychotic patients with PTSD showed trauma and abuse-associated FA decreases in the corpus callosum ${ }^{39}$. Although at baseline decreased FA was found in the corpus callosum in patients with a psychotic disorder (chapter 2), this WM tract was not examined in relation to childhood trauma as in the longitudinal tract-specific ROI analysis there was no evidence for a significant FA change over time, nor for a group effect on $\triangle \mathrm{FA}$ in the corpus callosum (chapter 3 ).

Nevertheless, both the corpus callosum and the cingulum have previously been found to be affected in healthy populations with stress experiences and in subjects with a clinical phenotype, such as schizophrenia, bipolar disorder or autism ${ }^{44}$.

In conclusion, a complicated interplay between traumatic impact and psychosis/ illness-related factors may be at hand causing an additive effect upon pre-onset microstructural WM changes in patients with psychotic disorder. Hypothetically, childhood trauma may lead to (chronic) stress and cingulum and corpus callosum alterations, which could contribute to the emergence or maintenance of psychiatric symptoms or syndromes, depending on the pre-existing (epi)genetic vulnerability. In turn, the symptom- or syndrome related stress may additionally and adversely impact these WM tracts.

\section{Cannabis}

Besides the effects of trauma, early cannabis use as a major biological trigger for a first psychotic episode has been the topic of the current thesis. Previously, studies have shown that adolescents who used cannabis had a two-fold risk of later developing schizophrenia ${ }^{45,46}$. However, the specific effect that cannabis may have on white matter is still unclear. One hypothesis is that cannabis, binding on the cannabinoid receptor CB1 at oligodendrocytes, may induce apoptosis of the oligodendrocyte progenitors, thereby affecting white matter development ${ }^{47}$. Evidence is however inconclusive. For example, reduced GM volume in the anterior cingulate gyrus ${ }^{48}$ and widespread reduced cortical thickness (CT) ${ }^{49}$, as well as no differences in brain morphology ${ }^{50,51}$ have been documented in patients with schizophrenia using cannabis compared to non-users. Habets and colleagues (2010) examining the same study sample, found cannabis-use associated decreased CT in patients with psychotic disorder as well as their unaffected siblings ${ }^{49}$. A longitudinal study showed additional cortical thinning in the left dorsolateral prefrontal cortex, left anterior cingulate cortex and left occipital lobe in cannabis using first episode patients compared to non-using patients with schizophrenia during a 5-year scan interval ${ }^{52}$. 


\section{Cannabis associated WM tract alterations}

DTI studies investigating WM microstructure in cannabis using non-psychotic patients are scarce, and often confounded by other drug use. A recent systematic review by Baker and colleagues (2013) included 10 studies, measuring diffusion indices in adolescent (non-psychotic) substance abusers (mainly alcohol and cannabis) compared to non-using controls. Microstructural WM alterations, generally a lower FA, were predominantly found in neocortical association pathways such as the superior longitudinal fasciculus and the corpus callosum in adolescent substance abusers ${ }^{53}$. Most studies found cannabis associated FA decreases, although FA increases have been detected, as well as absence of (cannabis related) white matter integrity loss ${ }^{54}$. This absence of effect was also seen in a longitudinal study with 41 mid-adolescent cannabis and alcohol abusers, showing higher diffusivity in the SLF associated with greater alcohol consumption, but not for cannabis use, after an 18-month follow-up ${ }^{55}$. However, Epstein \& Kumra (2015) reported that larger cannabis exposure during an 18 month scan interval predicted a greater FA decrease in the left inferior longitudinal fasciculus (ILF) in non-psychotic adolescents with a cannabis use disorder ( $n=19)$ compared to healthy adolescents $(n=29)^{56}$.

DTI studies investigating the effect of cannabis on WM integrity in patients with schizophrenia are even less available. The presented study in chapter 4 showed, in patients with psychotic disorder (but not siblings and controls), a whole-brain mean FA decline over a 3-year period associated with higher levels of cannabis exposure. The ROI analyses did not yield convincing evidence for regional WM alterations. The cross-sectional results at follow-up showed significant whole-brain mean FA decrease in the heavy cannabis using patients with a psychotic disorder as compared to siblings and controls. These findings of decreased FA concur with a cross-sectional study by James and colleagues (2011) that also showed FA decreases in brain stem, internal capsule, corona radiata, superior and inferior longitudinal fasciculus in cannabis using patients with psychotic disorder compared to non-using patients ${ }^{57}$. However, an earlier study found increased directional coherence in the bilateral uncinate fasciculus, the anterior part of the internal capsule and the frontal white matter in patients with a recent-onset psychotic episode, who had started to use cannabis before the age 17 , compared to healthy controls ${ }^{58}$.

Thus, diffusion studies examining the association of cannabis with brain white matter have described a variety of WM tracts, small effect sizes and contradictory directions of effect. These conflicting results may be explained by methodological differences, in analytical approach (voxel-based morphometry vs. ROI), and in sample characteristics. Differences in age of onset, disease stage (early onset, first-episode vs. subacute), symptom severity, as well as the age of onset (before and after age of 17), frequency of cannabis use, type/strength of cannabis and other drug use complicate the search 
for the underlying etiology of WM abnormalities in cannabis using patients with psychotic disorder. Nevertheless, from the findings in this thesis, one may hypothesize that cannabis exposure in a critical age-period may alter (accelerate) the normal trajectory of WM brain maturation in genetically vulnerable individuals. Interaction with illness-related factors may subsequently cause additional reduction in WM FA later in life. Whether this has clinical implications or influences long-term prognosis needs further investigation.

In conclusion, the patient-specific childhood trauma and cannabis associated FA decline provide further evidence for the role of environmental factors in cerebral white matter alterations associated with psychotic disorder, possibly in interaction with illness-related factors. In theory, altered connectivity may be an epiphenomenon, the resultant of developmentally altered wiring and / or aberrant synaptic plasticity 59 . The findings from the present thesis comply with aberrant WM connectivity that may have existed from or even before the early stages of the illness as well as with WM changes over time in a later phase of the illness, and depending on the level of environmental exposures. These environmental risk factors, along with other potential disease related factors (e.g. antipsychotic medication, reduced physical activity, social deprivation, smoking) may have led to an exceedance of the resilience capacity, thereby inducing an additional compromising effect on WM trajectory upon genetic vulnerability.

\section{Antipsychotic medication}

There is an ongoing debate on the effects of antipsychotic (AP) medication on brain tissue. Although results from animal studies and in-vivo studies suggest a protective effect of antipsychotics as they may promote (re)myelination ${ }^{60}$ and influence cell proliferation by modulating brain growth factors and cellular morphology ${ }^{61}$, there is also evidence for lower brain weight and volume associated with antipsychotic medication ${ }^{62}$. A recent review on the effect of AP medication on progressive brain changes in schizophrenia, based on 41 longitudinal structural imaging studies (1391 patients vs. 1135 controls), concluded that with regard to $\mathrm{CT}, \mathrm{GM}$ and WM volume, that results are still inconclusive ${ }^{63}$.

Although it was not one of the primary targets of this thesis, the effect of AP medication on brain structure (fractional anisotropy) was investigated in the patient sample. The cross-sectional results in chapter $\mathbf{2}$ did not yield a significant association between cumulative lifetime AP exposure and FA. In the longitudinal analysis, presented in chapter 3, a close to significant negative association was found between lifetime AP use and whole-brain mean $\triangle F A$, as well as a significant negative association between cumulative AP exposure over a 3-year interval and whole brain mean $\triangle F A$ over those 3 years. Especially in patients with the highest level of AP exposure, compared to 
controls, there was a significant decrease in FA over time that was not seen in the low and moderate AP exposure groups.

These results are in line with two other studies on this matter, showing FA decrease in the bilateral anterior cingulate gyrus and the right anterior corona radiata in firstepisode drug-naïve psychotic patients $(n=35)$ after 6 weeks of AP treatment ${ }^{64}$, and FA reductions in the parietal and occipital WM regions after 12 weeks of treatment with a second-generation antipsychotic in first-episode psychotic patients $(n=35)^{65}$. Four other studies showed opposite results: i) a positive association between amisulpride dosage and FA change in the right corticospinal tract in 28 first-episode psychotic patients after a 6 -week follow-up ${ }^{66}$, ii) a reduction in mean diffusivity within the right pyramidal tract, left temporal lobe, and cingulate gyrus after 28 days of antipsychotic use in 8 drug-free acutely psychotic patients who responded to the treatment ${ }^{67}$, iii) FA increase in mainly the uncinate, cingulum and corpus callosum in first-episode psychotic patients $(n=42)$ after 12 weeks of antipsychotic treatment, whereas FA also positively correlated with AP exposure ${ }^{68}$ and iv) widespread FA increase after 12 weeks on clozapine treatment in 16 patients with psychotic disorder ${ }^{69}$. In summary, half of these longitudinal DTI studies, using a pre-post treatment measurement, found a short-term (4-12 weeks) positive effect (FA increase) of AP medication on WM integrity in first-episode patients with psychotic disorder. This could fit the hypothesis that dopamine D2 blockers may reduce neurotoxic glutamate levels ${ }^{70}$ or have pro-myelinating effects, boosting myelin repair and oligodendrocyte differentiation ${ }^{71}$. The other half of the studies, including the one presented in chapter 3 and 4 with the longest between-scan interval ( 3 years), found FA reductions in various brain regions associated with (long term) AP exposure and a minor confounding effect of AP use on some of the interactions with environmental risk factors.

Overall, the evidence suggests that AP medication, like other environmental and illness-related factors, may contribute to microstructural WM alterations (increase and decrease),

The inconsistencies between the studies to date may have resulted from heterogeneous patient samples and differences in illness duration, AP type (first and second generation antipsychotics), cumulative lifetime AP dose assessment, and DTI processing techniques. Longitudinal (short, medium and long-term) studies using advanced diffusion imaging techniques, such as 'diffusion kurtosis imaging' or 'free water imaging' to examine respectively tissue characterization and putative signs for neuro-inflammation in more detail ${ }^{72}$, could help to further elucidate the impact of AP medication on neuronal (white matter) integrity. 


\section{Methodological considerations \& directions for future research}

The present thesis provides important new insights on WM structure in patients with a psychotic disorder and their unaffected siblings. However, one has to keep in mind that MRI only yields an indirect measure of brain structure and that diffusion imaging is influenced by several non-anatomical factors, requiring complex imaging processing and analysing techniques. Jones \& Cercignani (2010) have described the numerous steps in the processing pipeline with associated sources of bias that may lead to substantial errors ${ }^{73}$. There are also various confounders (e.g. head motion, changes in body weight, blood lipid levels, nicotine use, exercise, cortisol levels), often not taken into account, resulting in inconclusive findings ${ }^{74}$. Abovementioned sources of processing bias and confounders also apply to the DTI studies presented in this thesis, thus the results and conclusions in particular should be regarded with the necessary caution. Some (other) shortcomings of the presented studies in this thesis are discussed below with recommendations for future research.

\section{DTI measure}

The significance of the frequently reported cross-sectional and longitudinal findings of FA decreases is still unclear. FA is a rather non-specific indirect diffusion measure on 'neuronal integrity', not necessarily referring to a damaging or degenerative process, when applied in other contexts (e.g. intelligence, creativity) ${ }^{75}$. Secondary measures as radial diffusivity for the degree of myelination and axial diffusivity for axonal organization are more informative on the etiology of WM alterations in terms of myelin loss or axonal incoherence but as yet, less frequently reported. Lately, as it is apparent that the structure of neural tissue may be too complex to comprehend through a single diffusion tensor, the focus has shifted to more advanced WM measures derived from 'Free Water Imaging (FWI)' and the 'Permeability Diffusivity Index (PDI) ${ }^{72}$. Future studies examining WM integrity should implement these new diffusion methods to investigate the nature of the alterations in WM microstructure in more detail.

\section{Sample size}

Sample size and sample characteristics in neuroimaging studies are an important issue to take into account. Despite a relatively large sample size of the presented studies in this thesis compared to other diffusion studies, false positive results may be at hand. A recent study by Melicher and colleagues (2015) showed that detected regional FA differences strongly depend on the size of the studied sample. Studies with small samples are at risk for falsely suggesting WM abnormalities in certain re- 
gions ${ }^{76}$. Therefore, a minimum sample size for diffusion imaging should be imposed, especially if subgroups or statistical interactions are analysed.

\section{Sample characteristics}

Diffusion imaging studies have reported WM abnormalities in a variety of brain regions ${ }^{77}$ that have considerable overlap with regions detected in other psychiatric disorders, such as autism ${ }^{78}$ and bipolar disorder ${ }^{79}$. This may indicate that these WM alterations may not be disorder-specific, but rather reflecting trans-diagnostic symptom dimensions. The majority of the DTI studies included first-episode or chronic patients with psychotic disorder, based on DSM-IV criteria, not accounting for individual fluctuations or presence of specific psychotic symptoms, subgroup-variations in illness severity, environmental (e.g. birth-complications, childhood trauma, cannabis use) and lifestyle factors (BMI, smoking, physical activity). More consistent findings are expected if future patient samples are compiled based on symptoms profiles and aforementioned patient-specific factors, and not solemnly on arbitrary (DSM-IV or DSM-5 based) diagnostic categories ${ }^{80}$. The National Institute of Mental Health already adopted this approach defining the Research Domain Criteria (RDoC) framework, studying underlying functional constructs across different psychiatric disorders ${ }^{81}$.

\section{Combining DTI with resting state fMRI}

The frequently reported microstructural WM alterations in patients with a psychotic disorder ${ }^{82}$ correspond to various findings from functional connectivity studies, mainly showing alterations in the fronto-temporal brain region ${ }^{83}$, the fronto-striatal circuit ${ }^{84}$ and the cingulo-opercular system, which is involved in interoception and salience processing ${ }^{85}$. In order to investigate whether altered diffusivity ('damage') within relevant WM networks may be associated with abnormal neural activation ('dysfunction'), studies combining diffusion imaging with resting-state functional MRI have been performed. The results showed associations between functional and structural connectivity ${ }^{87}$, in particular for the cingulum tract, interconnecting the key regions of the default mode network ${ }^{88}$. As resting-state scans were acquired and analysed of the current sample ${ }^{86}$, it may be worthwhile to further explore such structure-functions associations in the near future.

\section{Conclusion}

This thesis investigated microstructural white matter alterations in patients with a psychotic disorder, unaffected siblings of patients with psychotic disorder and healthy controls. Patients showed: i) widespread reduced FA compared to siblings and con- 
trols, ii) a relatively stable whole brain FA over the 3-year follow-up period, and iii) a significant steeper decline in FA associated with higher levels of cannabis exposure and childhood trauma, as compared to siblings and controls. Siblings showed: i) no significant FA differences at baseline, ii) a significant mean FA decline (whole brain, in the right cingulum and in the left PTR) and a smaller FA increase in the right RPIC over the 3-year follow-up period, iii) a significant difference with controls in whole brain mean FA at follow-up, and iv) absence of cannabis and childhood trauma associated FA change over time. These findings partly comply with neurodevelopmental global and regional white matter alterations compromising connectivity in the early stage of psychotic disorder and in subjects at higher than average genetic risk. Genes and environment may not only influence neurodevelopmental processes, but also impact neuroplasticity later in life, possibly in interaction with illness-related factors. 


\section{References}

1. van Os J, Kenis G, Rutten BP. The environment and schizophrenia. Nature Nov 11 2010;468(7321):203-212.

2. Gottesman, II, Gould TD. The endophenotype concept in psychiatry: etymology and strategic intentions. The American journal of psychiatry Apr 2003;160(4):636-645.

3. Kendler KS, Neale MC. Endophenotype: a conceptual analysis. Molecular psychiatry Aug 2010;15(8):789-797.

4. Pantelis C, Wood SJ, Velakoulis D, Testa R, Fontenelle LF, Yucel M. Should we redefine the concept of endophenotype in schizophrenia? Rev Bras Psiquiatr Jun 2010;32(2):106-107.

5. Arat HE, Chouinard VA, Cohen BM, Lewandowski KE, Ongur D. Diffusion tensor imaging in first degree relatives of schizophrenia and bipolar disorder patients. Schizophrenia research Feb 2015;161(2-3):329-339.

6. Boos HB, Mandl RC, van Haren NE, Cahn W, van Baal GC, Kahn RS, Hulshoff Pol HE. Tract-based diffusion tensor imaging in patients with schizophrenia and their non-psychotic siblings. Eur Neuropsychopharmacol Apr 2013;23(4):295-304.

7. Camchong J, Lim KO, Sponheim SR, Macdonald AW. Frontal white matter integrity as an endophenotype for schizophrenia: diffusion tensor imaging in monozygotic twins and patients' nonpsychotic relatives. Front Hum Neurosci 2009;3:35.

8. Domen PA, Michielse S, Gronenschild E, Habets P, Roebroeck A, Schruers K, van Os J, Marcelis M. Microstructural white matter alterations in psychotic disorder: A family-based diffusion tensor imaging study. Schizophrenia research May 2013;146(1-3):291-300.

9. Goghari VM, Billiet T, Sunaert S, Emsell L. A diffusion tensor imaging family study of the fornix in schizophrenia. Schizophrenia research Nov 2014;159(2-3):435-440.

10. Knochel C, O'Dwyer L, Alves G, et al. Association between white matter fiber integrity and subclinical psychotic symptoms in schizophrenia patients and unaffected relatives. Schizophrenia research Sep 2012;140(1-3):129-135.

11. Munoz Maniega S, Lymer GK, Bastin ME, et al. A diffusion tensor MRI study of white matter integrity in subjects at high genetic risk of schizophrenia. Schizophrenia research Dec 2008;106(2-3):132-139.

12. Phillips $\mathrm{OR}$, Nuechterlein $\mathrm{KH}$, Asarnow RF, et al. Mapping corticocortical structural integrity in schizophrenia and effects of genetic liability. Biological psychiatry Oct 1 2011;70(7):680-689.

13. Prasad KM, Upton CH, Schirda CS, Nimgaonkar VL, Keshavan MS. White matter diffusivity and microarchitecture among schizophrenia subjects and first-degree relatives. Schizophrenia research Jan 2015;161(1):70-75.

14. Hao Y, Yan Q, Liu H, et al. Schizophrenia patients and their healthy siblings share disruption of white matter integrity in the left prefrontal cortex and the hippocampus but not the anterior cingulate cortex. Schizophrenia research Oct 2009;114(1-3):128-135.

15. Hoptman MJ, Nierenberg J, Bertisch HC, Catalano D, Ardekani BA, Branch CA, Delisi LE. A DTI study of white matter microstructure in individuals at high genetic risk for schizophrenia. Schizophrenia research Dec 2008;106(2-3):115-124.

16. Clark KA, Nuechterlein KH, Asarnow RF, et al. Mean diffusivity and fractional anisotropy as indicators of disease and genetic liability to schizophrenia. J Psychiatr Res Jul 2011;45(7):980988. 
17. Knochel C, Oertel-Knochel V, Schonmeyer R, et al. Interhemispheric hypoconnectivity in schizophrenia: fiber integrity and volume differences of the corpus callosum in patients and unaffected relatives. Neurolmage Jan 16 2012;59(2):926-934.

18. Imperati D, Colcombe S, Kelly C, Di Martino A, Zhou J, Castellanos FX, Milham MP. Differential development of human brain white matter tracts. PloS one 2011;6(8):e23437.

19. van den Heuvel MP, Fornito A. Brain networks in schizophrenia. Neuropsychology review Mar 2014;24(1):32-48.

20. Zalesky A, Fornito A, Seal ML, Cocchi L, Westin CF, Bullmore ET, Egan GF, Pantelis C. Disrupted axonal fiber connectivity in schizophrenia. Biological psychiatry Jan 01 2011;69(1):80-89.

21. Segal D, Haznedar MM, Hazlett EA, et al. Diffusion tensor anisotropy in the cingulate gyrus in schizophrenia. Neurolmage Apr 1 2010;50(2):357-365.

22. Abdul-Rahman MF, Qiu A, Sim K. Regionally specific white matter disruptions of fornix and cingulum in schizophrenia. PloS one 2011;6(4):e18652.

23. Sun Z, Wang F, Cui L, et al. Abnormal anterior cingulum in patients with schizophrenia: a diffusion tensor imaging study. Neuroreport Oct 6 2003;14(14):1833-1836.

24. Wang F, Sun Z, Cui L, et al. Anterior cingulum abnormalities in male patients with schizophrenia determined through diffusion tensor imaging. The American journal of psychiatry Mar 2004;161(3):573-575.

25. Wheeler AL, Voineskos AN. A review of structural neuroimaging in schizophrenia: from connectivity to connectomics. Front Hum Neurosci 2014;8:653.

26. Mitelman SA, Canfield EL, Newmark RE, et al. Longitudinal Assessment of Gray and White Matter in Chronic Schizophrenia: A Combined Diffusion-Tensor and Structural Magnetic Resonance Imaging Study. The open neuroimaging journal 2009;3:31-47.

27. Tang J, Liao Y, Zhou B, Tan C, Liu T, Hao W, Hu D, Chen X. Abnormal anterior cingulum integrity in first episode, early-onset schizophrenia: a diffusion tensor imaging study. Brain research Jul 9 2010;1343:199-205.

28. Hoptman MJ, Ardekani BA, Butler PD, Nierenberg J, Javitt DC, Lim KO. DTI and impulsivity in schizophrenia: a first voxelwise correlational analysis. Neuroreport Nov 15 2004;15(16):24672470.

29. Fujiwara H, Namiki C, Hirao K, et al. Anterior and posterior cingulum abnormalities and their association with psychopathology in schizophrenia: a diffusion tensor imaging study. Schizophrenia research Sep 2007;95(1-3):215-222.

30. Peters BD, de Haan L, Dekker $\mathrm{N}$, et al. White matter fibertracking in first-episode schizophrenia, schizoaffective patients and subjects at ultra-high risk of psychosis. Neuropsychobiology 2008;58(1):19-28.

31. Vogt BA. Pain and emotion interactions in subregions of the cingulate gyrus. Nature reviews Neuroscience Jul 2005;6(7):533-544.

32. Smith MJ, Barch DM, Thompson PA, Csernansky JG. Subclinical expression of schizophrenialike symptoms in non-psychotic siblings of individuals with schizophrenia. Schizophrenia research Aug 2008;103(1-3):324-325.

33. Ohi K, Hashimoto R, Yasuda $Y$, et al. Impact of the genome wide supported NRGN gene on anterior cingulate morphology in schizophrenia. PloS one 2012;7(1):e29780.

34. Kuswanto CN, Woon PS, Zheng XB, et al. Genome-wide supported psychosis risk variant in ZNF804A gene and impact on cortico-limbic WM integrity in schizophrenia. American journal of medical genetics Part B, Neuropsychiatric genetics : the official publication of the International Society of Psychiatric Genetics Apr 2012;159B(3):255-262. 
35. van den Heuvel MP, Hulshoff Pol HE. Exploring the brain network: a review on resting-state fMRI functional connectivity. Eur Neuropsychopharmacol Aug 2010;20(8):519-534.

36. Chiang MC, McMahon KL, de Zubicaray GI, Martin NG, Hickie I, Toga AW, Wright MJ, Thompson PM. Genetics of white matter development: a DTI study of 705 twins and their siblings aged 12 to 29. Neurolmage Feb 1 2011;54(3):2308-2317.

37. Teicher $\mathrm{MH}$, Samson JA, Anderson CM, Ohashi K. The effects of childhood maltreatment on brain structure, function and connectivity. Nature reviews Neuroscience Sep 19 2016;17(10):652-666.

38. van der Werff SJ, van den Berg SM, Pannekoek JN, Elzinga BM, van der Wee NJ. Neuroimaging resilience to stress: a review. Frontiers in behavioral neuroscience 2013;7:39.

39. Daniels JK, Lamke JP, Gaebler M, Walter H, Scheel M. White matter integrity and its relationship to PTSD and childhood trauma--a systematic review and meta-analysis. Depression and anxiety Mar 2013;30(3):207-216.

40. Dedovic K, D’Aguiar C, Pruessner JC. What stress does to your brain: a review of neuroimaging studies. Canadian journal of psychiatry Revue canadienne de psychiatrie Jan 2009;54(1):6-15.

41. Bremner JD. Functional neuroimaging in post-traumatic stress disorder. Expert review of neurotherapeutics Apr 2007;7(4):393-405.

42. Catani M, Jones DK, Donato R, Ffytche DH. Occipito-temporal connections in the human brain. Brain : a journal of neurology Sep 2003;126(Pt 9):2093-2107.

43. Bauer IE, Ouyang A, Mwangi B, Sanches M, Zunta-Soares GB, Keefe RS, Huang H, Soares JC. Reduced white matter integrity and verbal fluency impairment in young adults with bipolar disorder: a diffusion tensor imaging study. J Psychiatr Res Mar 2015;62:115-122.

44. Ameis SH, Lerch JP, Taylor MJ, et al. A Diffusion Tensor Imaging Study in Children With ADHD, Autism Spectrum Disorder, OCD, and Matched Controls: Distinct and Non-Distinct White Matter Disruption and Dimensional Brain-Behavior Relationships. The American journal of psychiatry Jul 1 2016;173(12), 1213-1222.

45. Arseneault L, Cannon M, Witton J, Murray RM. Causal association between cannabis and psychosis: examination of the evidence. Br J Psychiatry Feb 2004;184:110-117.

46. Henquet C, Krabbendam L, Spauwen J, Kaplan C, Lieb R, Wittchen HU, van Os J. Prospective cohort study of cannabis use, predisposition for psychosis, and psychotic symptoms in young people. Bmj Jan 1 2005;330(7481):11.

47. Molina-Holgado E, Vela JM, Arevalo-Martin A, Almazan G, Molina-Holgado F, Borrell J, Guaza C. Cannabinoids promote oligodendrocyte progenitor survival: involvement of cannabinoid receptors and phosphatidylinositol-3 kinase/Akt signaling. The Journal of neuroscience : the official journal of the Society for Neuroscience Nov 15 2002;22(22):9742-9753.

48. Szeszko PR, Robinson DG, Sevy S, et al. Anterior cingulate grey-matter deficits and cannabis use in first-episode schizophrenia. Br J Psychiatry Mar 2007;190:230-236.

49. Habets $\mathrm{P}$, Marcelis M, Gronenschild E, Drukker M, van Os J. Reduced cortical thickness as an outcome of differential sensitivity to environmental risks in schizophrenia. Biological psychiatry Mar 1 2010;69(5):487-494.

50. Cahn W, Hulshoff Pol HE, Caspers E, van Haren NE, Schnack HG, Kahn RS. Cannabis and brain morphology in recent-onset schizophrenia. Schizophrenia research Apr 1 2004;67(2-3):305307.

51. Wobrock T, Sittinger H, Behrendt B, D'Amelio R, Falkai P. Comorbid substance abuse and brain morphology in recent-onset psychosis. European archives of psychiatry and clinical neuroscience Feb 2009;259(1):28-36. 
52. Rais M, van Haren NE, Cahn W, et al. Cannabis use and progressive cortical thickness loss in areas rich in CB1 receptors during the first five years of schizophrenia. Eur Neuropsychopharmacol Dec 2010;20(12):855-865.

53. Baker ST, Yucel M, Fornito A, Allen NB, Lubman DI. A systematic review of diffusion weighted MRI studies of white matter microstructure in adolescent substance users. Neuroscience and biobehavioral reviews Sep 2013;37(8):1713-1723.

54. Delisi LE, Bertisch HC, Szulc KU, Majcher M, Brown K, Bappal A, Ardekani BA. A preliminary DTI study showing no brain structural change associated with adolescent cannabis use. Harm Reduct J 2006;3:17.

55. Bava S, Jacobus J, Thayer RE, Tapert SF. Longitudinal changes in white matter integrity among adolescent substance users. Alcoholism, clinical and experimental research Jan 2013;37 Suppl 1:E181-189.

56. Epstein KA, Kumra S. White matter fractional anisotropy over two time points in early onset schizophrenia and adolescent cannabis use disorder: A naturalistic diffusion tensor imaging study. Psychiatry research Apr 30 2015;232(1):34-41.

57. James A, Hough M, James S, Winmill L, Burge L, Nijhawan S, Matthews PM, Zarei M. Greater white and grey matter changes associated with early cannabis use in adolescent-onset schizophrenia (AOS). Schizophrenia research May 2011;128(1-3):91-97.

58. Peters BD, de Haan L, Vlieger EJ, Majoie CB, den Heeten GJ, Linszen DH. Recent-onset schizophrenia and adolescent cannabis use: MRI evidence for structural hyperconnectivity? Psychopharmacol Bull 2009;42(2):75-88.

59. Stephan KE, Friston KJ, Frith CD. Dysconnection in schizophrenia: from abnormal synaptic plasticity to failures of self-monitoring. Schizophrenia bulletin May 2009;35(3):509-527.

60. Walterfang M, Velakoulis D, Whitford TJ, Pantelis C. Understanding aberrant white matter development in schizophrenia: an avenue for therapy? Expert review of neurotherapeutics Jul 2011;11(7):971-987.

61. Crespo-Facorro B, Roiz-Santianez R, Perez-Iglesias R, et al. Effect of antipsychotic drugs on brain morphometry. A randomized controlled one-year follow-up study of haloperidol, risperidone and olanzapine. Prog Neuropsychopharmacol Biol Psychiatry Dec 12 2008;32(8):19361943.

62. Dorph-Petersen KA, Pierri JN, Perel JM, Sun Z, Sampson AR, Lewis DA. The influence of chronic exposure to antipsychotic medications on brain size before and after tissue fixation: a comparison of haloperidol and olanzapine in macaque monkeys. Neuropsychopharmacology Sep 2005;30(9):1649-1661.

63. Roiz-Santianez R, Suarez-Pinilla P, Crespo-Facorro B. Brain Structural Effects of Antipsychotic Treatment in Schizophrenia: A Systematic Review. Curr Neuropharmacol 2015;13(4):422-434.

64. Wang $Q$, Cheung $C$, Deng $W$, et al. White-matter microstructure in previously drug-naive patients with schizophrenia after 6 weeks of treatment. Psychol Med Nov 2013;43(11):23012309.

65. Szeszko PR, Robinson DG, Ikuta T, Peters BD, Gallego JA, Kane J, Malhotra AK. White matter changes associated with antipsychotic treatment in first-episode psychosis. Neuropsychopharmacology May 2014;39(6):1324-1331.

66. Ebdrup BH, Raghava JM, Nielsen MO, Rostrup E, Glenthoj B. Frontal fasciculi and psychotic symptoms in antipsychotic-naive patients with schizophrenia before and after 6 weeks of selective dopamine D2/3 receptor blockade. Journal of psychiatry \& neuroscience : JPN Mar 2016;41(2):133-141. 
67. Garver DL, Holcomb JA, Christensen JD. Compromised myelin integrity during psychosis with repair during remission in drug-responding schizophrenia. Int J Neuropsychopharmacol Feb 2008;11(1):49-61.

68. Reis Marques $\mathrm{T}$, Taylor $\mathrm{H}$, Chaddock $\mathrm{C}$, et al. White matter integrity as a predictor of response to treatment in first episode psychosis. Brain : a journal of neurology Jan 2014;137(Pt 1):172182.

69. Ozcelik-Eroglu E, Ertugrul A, Oguz KK, Has AC, Karahan S, Yazici MK. Effect of clozapine on white matter integrity in patients with schizophrenia: a diffusion tensor imaging study. Psychiatry research Sep 30 2014;223(3):226-235.

70. Paz RD, Tardito S, Atzori M, Tseng KY. Glutamatergic dysfunction in schizophrenia: from basic neuroscience to clinical psychopharmacology. Eur Neuropsychopharmacol Nov 2008;18(11):773-786.

71. Bartzokis G. Neuroglialpharmacology: myelination as a shared mechanism of action of psychotropic treatments. Neuropharmacology Jun 2012;62(7):2137-2153.

72. Karlsgodt KH. Diffusion Imaging of White Matter In Schizophrenia: Progress and Future Directions. Biol Psychiatry Cogn Neurosci Neuroimaging May 2016;1(3):209-217.

73. Jones DK, Cercignani M. Twenty-five pitfalls in the analysis of diffusion MRI data. NMR in biomedicine Aug 2010;23(7):803-820.

74. Weinberger DR, Radulescu E. Finding the Elusive Psychiatric "Lesion" With 21st-Century Neuroanatomy: A Note of Caution. The American journal of psychiatry Jan 2016;173(1):27-33.

75. Jones DK, Knosche TR, Turner R. White matter integrity, fiber count, and other fallacies: the do's and don'ts of diffusion MRI. Neurolmage Jun 2013;73:239-254.

76. Melicher T, Horacek J, Hlinka J, et al. White matter changes in first episode psychosis and their relation to the size of sample studied: a DTI study. Schizophrenia research Mar 2015;162(13):22-28.

77. Samartzis L, Dima D, Fusar-Poli P, Kyriakopoulos M. White Matter Alterations in Early Stages of Schizophrenia: A Systematic Review of Diffusion Tensor Imaging Studies. J Neuroimaging 2014 Mar-Apr;24(2):101-10.

78. Travers BG, Adluru N, Ennis C, et al. Diffusion tensor imaging in autism spectrum disorder: a review. Autism research : official journal of the International Society for Autism Research Oct 2012;5(5):289-313.

79. Nortje G, Stein DJ, Radua J, Mataix-Cols D, Horn N. Systematic review and voxel-based metaanalysis of diffusion tensor imaging studies in bipolar disorder. Journal of affective disorders Sep 5 2013;150(2):192-200.

80. Linden DE. The challenges and promise of neuroimaging in psychiatry. Neuron Jan 12 2012;73(1):8-22.

81. Insel T, Cuthbert B, Garvey M, Heinssen R, Pine DS, Quinn K, Sanislow C, Wang P. Research domain criteria (RDoC): toward a new classification framework for research on mental disorders. The American journal of psychiatry Jul 2010;167(7):748-751.

82. Ellison-Wright I, Bullmore E. Meta-analysis of diffusion tensor imaging studies in schizophrenia. Schizophrenia research Mar 2009;108(1-3):3-10.

83. Hoffman RE, Fernandez T, Pittman B, Hampson M. Elevated functional connectivity along a corticostriatal loop and the mechanism of auditory/verbal hallucinations in patients with schizophrenia. Biological psychiatry Mar 1 2011;69(5):407-414.

84. Fornito A, Harrison BJ, Goodby E, et al. Functional dysconnectivity of corticostriatal circuitry as a risk phenotype for psychosis. JAMA psychiatry Nov 2013;70(11):1143-1151. 
85. Palaniyappan L, Simmonite M, White TP, Liddle EB, Liddle PF. Neural primacy of the salience processing system in schizophrenia. Neuron Aug 21 2013;79(4):814-828.

86. Peeters SC, Gronenschild EH, van de Ven V, et al. Altered mesocorticolimbic functional connectivity in psychotic disorder: an analysis of proxy genetic and environmental effects. Psychol Med Jul 2015;45(10):2157-2169.

87. Koch MA, Norris DG, Hund-Georgiadis M. An investigation of functional and anatomical connectivity using magnetic resonance imaging. Neurolmage May 2002;16(1):241-250.

88. van den Heuvel M, Mandl R, Luigjes J, Hulshoff Pol H. Microstructural organization of the cingulum tract and the level of default mode functional connectivity. The Journal of neuroscience : the official journal of the Society for Neuroscience Oct 22 2008;28(43):10844-10851. 



\title{
Summary
}

\section{Samenvatting}

\author{
Valorisation
}

Dankwoord

Curriculum Vitae

List of Publications 

Cerebral vulnerability, characterized by altered neural communication between various brain regions, is assumed to be one of the factors that contribute to the etiology of psychotic disorder. This structural dysconnectivity, possibly already occurring before illness-onset, may be caused by a complex interplay between genetic vulnerability and environmental risk factors during different stages of life. Diffusion tensor imaging (DTI) is a commonly applied method for investigating white matter (WM) structural connectivity in vivo. DTI is used in this thesis to investigate a potential WM intermediate phenotype for psychosis, to examine the course of WM development over time, and to explore the association between environmental stressors and WM change over time.

In chapter 1, a general introduction on the themes of this thesis, as well as the outline and aims of the thesis are described.

In chapter $\mathbf{2}$ a WM intermediate phenotype for psychosis is investigated. Although previous DTI studies in patients with a psychotic disorder have undisputedly shown a decrease in FA in fronto-temporal and fronto-occipital connections, a limited number of DTI studies, often with smaller sample sizes, have been performed on individuals at higher-than-average genetic risk for psychosis. In the current study, significantly lower mean FA in patients with a psychotic disorder compared to healthy controls was found throughout the brain, especially in the corpus callosum, forceps major and minor and several parts of the corona radiata. Intermediate values were found in healthy siblings with respect to patients and controls, yet no significant differences. Additional analyses failed to show a clear association between microstructural WM and antipsychotic medication. No confounding effect of cannabis, and only a minor confounding effect of other drug use, on microstructural WM was found. Furthermore, it was examined whether the WM group differences were sex dependent. Results of this analysis showed that the group differences between patients and controls may be partly due to lower FA values in the female participants. In conclusion, the finding of widespread WM abnormalities in patients with a psychotic disorder may reflect illness-related brain pathology, not providing clear evidence for an intermediate phenotype.

In chapter 3, microstructural white matter is examined over time in patients with a psychotic disorder, unaffected siblings and controls, by analyses of diffusion scans that were acquired at two time-points with, on average, a three year interval. The presented longitudinal study showed a relatively stable whole-brain mean FA in patients with psychotic disorder, opposed to a significant FA decline in unaffected siblings with respect to controls. WM tract-specific analyses revealed more tracts with a decrease than an increase over time in both patients and siblings. In particular, a smaller mean 
FA increase in the retrolenticular part of the internal capsule (RPIC), and a mean FA decrease in the posterior corona radiata ( $P C R$ ) and the cingulum (all right-sided) was found in patients with respect to controls. In siblings, a significant smaller mean FA increase in the right RPIC and a mean FA decrease in the right cingulum was found with respect to controls. Thus, in contrast to baseline, at follow-up both patients and siblings had a lower whole-brain mean FA with respect to controls. The results provide evidence for a process of WM alterations in individuals with liability for psychotic disorder. Moreover, decreased FA in patients with respect to controls may stabilize over time, without (further) WM decline, at least over a certain period during the course of the illness.

Numerous epidemiological studies point to environmental risk factors such as adolescent cannabis use and childhood trauma as the most common risk factors for psychotic disorder. In chapter 4 a cross-sectional as well as a longitudinal design was applied to examine the association between these environmental exposures and white matter alterations in patients with a psychotic disorder, their healthy siblings and a healthy control group. In contrast with the cross-sectional analysis at baseline, the follow-up analysis showed a significant negative association between cannabis use / childhood trauma and FA in patients with a psychotic disorder. This concurred with the longitudinal analysis, in which a larger whole-brain mean FA decline over 3 years was associated with higher levels of cannabis exposure and higher levels of childhood trauma in patients with a psychotic disorder. This pattern of results was not seen in the siblings and controls. In additional analyses on specific WM tracts, it was found that the childhood trauma exposure was associated with white matter alteration in the right cingulum, a stress-processing related region. This is the first longitudinal DTI study showing that the extent of microstructural WM alterations in patients with a psychotic disorder (but not in siblings and controls) is conditional on the level of environmental risk exposure, suggesting interactions between the environment and illness-related factors.

In chapter 5, the findings presented in the previous chapters are discussed into more detail and integrated from a wider perspective. In addition, strengths and weaknesses of the presented studies in this thesis are highlighted, providing directions for future DTI studies. 
Summary

Samenvatting

\author{
Valorisation \\ Dankwoord
}

Curriculum Vitae

List of Publications 

Cerebrale kwetsbaarheid, gekenmerkt door een veranderde neuronale communicatie tussen verschillende hersengebieden, wordt verondersteld een van de factoren te zijn die bijdraagt aan het ontstaan van een psychotische stoornis. Deze structurele disconnectiviteit, mogelijk al aanwezig voor aanvang van de aandoening, is het gevolg van een samenspel van genetische kwetsbaarheid en omgevingsrisicofactoren die zich door verschillende levensfasen heen voordoen. Diffusion Tensor Imaging (DTI) is een veelvuldig toegepaste in vivo methode om de mate van structurele connectiviteit van de witte stof te onderzoeken. DTI wordt in dit proefschrift toegepast om een mogelijk witte stof endofenotype voor psychose op te sporen, witte stof ontwikkeling over een bepaalde tijdsinterval te volgen, en te onderzoeken of er een associatie is tussen omgevingsstressoren en witte stof afwijkingen over de tijd.

In hoofdstuk 1 wordt na een algemene inleiding over de thema's in dit proefschrift, de opbouw en doelstellingen van het proefschrift besproken.

In hoofdstuk 2 wordt een witte stof endofenotype voor psychose onderzocht. Hoewel tal van DTI studies in patiënten met een psychotische stoornis een fractionele anisotropie (FA) daling in fronto-temporale en fronto-occipitale witte stof verbindingen hebben aangetoond, zijn er slechts een beperkt aantal DTI studies uitgevoerd, vaak met kleinere aantallen proefpersonen, bij gezonde eerstegraads familieleden. In het gepresenteerde onderzoek worden er significant lagere FA waarden gevonden, verspreid in het brein, vooral in het corpus callosum, forceps major en minor en verschillende onderdelen van de corona radiata, bij patiënten met een psychotische stoornis vergeleken met gezonde controle personen. Intermediaire afwijkingen, weliswaar zonder significante verschillen, worden gevonden bij gezonde broers/zussen, in vergelijking met de patiënten en de controles. Aanvullende analyses laten geen duidelijk associatie zien tussen witte stof veranderingen en antipsychotica gebruik en wordt er geen confounding effect van cannabis en slechts een gering confounding effect van andere (hard)drugs op de witte stof waargenomen.

Daarnaast is onderzocht of de gevonden groepsverschillen in witte stof afhankelijk zijn van geslacht. Resultaten laten zien dat het groepsverschil tussen controles en patiënten mogelijk deels wordt veroorzaakt door lagere FA waarden van de vrouwelijke proefpersonen. Kortom, de bevinding van wijdverspreide witte stof afwijkingen bij patiënten met een psychotische stoornis weerspiegelen mogelijke ziekte-gerelateerde breinpathologie, waarbij er geen duidelijke aanwijzingen zijn voor een endofenotype.

In hoofdstuk 3 wordt de witte stof ontwikkeling over een bepaalde tijdsinterval onderzocht bij patiënten met een psychotische stoornis, hun gezonde broers/zussen en een gezonde controlegroep. De gepresenteerde longitudinale studie toont over een 
periode van ongeveer 3 jaar, een relatief stabiele FA van het gehele brein bij patiënten met een psychotische stoornis, in tegenstelling tot een significante FA daling bij de broers/zussen, vergeleken met de gezonde controle groep. Een analyse gericht op de individuele witte stof vezelbundels toont aan dat meer bundels een FA daling laten zien i.p.v. een stijging bij zowel patiënten als hun gezonde eerstegraads familieleden, vergeleken met de gezonde controle personen. Er wordt een significant kleinere FA stijging gevonden van het retrolenticulaire deel van de capsula interna (RPIC), en een significante FA daling van de corona radiata posterior en het cingulum (allen rechtszijdig) bij de patiënten vergeleken met de controles. Bij de gezonde broers/zussen wordt ook een significant kleinere FA stijging gevonden van de rechter RPIC en FA daling van het rechter cingulum, wederom vergeleken met gezonde controles. In tegenstelling tot bij de nulmeting (hoofdstuk 2), wordt nu bij de follow-up een lagere FA van het gehele brein gevonden bij zowel patiënten met een psychotische stoornis als hun broers/zussen, vergeleken met de controle groep. Dit resultaat duidt niet alleen op een verstoorde ontwikkeling van de witte stof bij mensen met een kwetsbaarheid voor een psychotische stoornis, maar suggereert ook een stabilisatie van witte stof achteruitgang na ontstaan van de psychotische stoornis, althans gedurende een bepaalde periode in het ziektebeloop.

Talrijke epidemiologische studies hebben aangetoond dat omgevingsrisicofactoren, zoals trauma in de kindertijd en cannabisgebruik in de adolescentie, veel voorkomende risicofactoren voor psychose zijn. In hoofdstuk 4 wordt door middel van zowel een cross-sectionele als een longitudinale analyse, de associatie onderzocht tussen deze omgevingsstressoren en microstructurele witte stof veranderingen in patiënten met een psychotische stoornis, hun gezonde broers/zussen en een gezonde controle groep. In tegenstelling tot bij de nulmeting, worden bij de cross-sectionele vervolgmeting significante associaties gevonden tussen blootstelling aan cannabis / jeugdtrauma en FA bij patiënten met een psychotische stoornis. Dit komt overeen met de resultaten van de longitudinale analyse, waaruit blijkt dat een grotere FA daling in 3 jaar (gemiddeld over het gehele brein) is geassocieerd met een hogere blootstelling aan cannabis en jeugdtrauma bij patiënten met een psychotische stoornis. Dit patroon is afwezig bij de eerstegraads familieleden van deze patiënten en gezonde controles. In een aanvullende analyse gericht op veranderingen in specifieke witte stof vezelbundels wordt gevonden dat blootstelling aan trauma in de kindertijd geassocieerd blijkt te zijn met witte stof afwijkingen in een gebied betrokken bij de stress-verwerking; het rechter cingulum. Dit is de eerste longitudinale DTI studie waaruit blijkt dat de omvang van de microstructurele witte stof veranderingen bij patiënten met een psychotische stoornis (maar niet bij broers/ zussen en controles) 
afhankelijk is van de mate van blootstelling aan omgevingsrisicofactoren, en daarmee een interactie suggereren met omgeving en ziekte-gerelateerde factoren.

In hoofdstuk $\mathbf{5}$ worden de bevindingen uit de voorgaande hoofdstukken meer in detail besproken en geïntegreerd vanuit een breder perspectief. Daarnaast worden de sterke en zwakke punten van de gepresenteerde studies in dit proefschrift belicht, waarbij aangrijpingspunten voor toekomstig DTI studies worden benoemd. 

Summary

Samenvatting

Valorisation

Dankwoord

Curriculum Vitae

List of Publications 

Psychotic disorders are one of the leading causes worldwide of long-term disability. It is a psychiatric syndrome consisting of perceptual, cognitive, emotional and behavioural symptoms. The prevalence rate is relatively low $( \pm 3 \%$ for the broader psychosis spectrum) and remission can be achieved for the majority of the patients. However, a reasonable proportion of patients with a psychotic disorder experience persisting social and professional impairments in daily life. The cost for psychotic disorders is still enormous, estimated almost 94 billion euros in 2010 in Europe, although the ratio of societal cost per patient to 2015 gross domestic product per capita varies from $37 \%$ in Switzerland to $214 \%$ in the UK ${ }^{1}$. Expenses are related to long-term unemployment, and high costs of chronic use of treatment and support. The etiology of the disease is still largely unknown and the current treatment is not curative, merely focused on attenuating the primary symptoms ${ }^{2,3}$.

\section{Science: new diffusion parameters, replication and other environmental risk factors}

The presented studies in this thesis yielded several insights on the course of white matter (WM) integrity in patients with a psychotic disorder and their genetically related healthy siblings. One of the studies of this thesis showed widespread crosssectional patient-control differences in fractional anisotropy (FA), while siblings of patients revealed intermediate FA values, although not significant from the control group. These findings teach us that these WM alterations may reflect illness, or treatment related effects rather than a genetic risk. Future studies examining WM integrity should implement new diffusion methods as 'Free Water Imaging (FWI)' and the 'Permeability Diffusivity Index (PDI) to investigate the nature of these alterations in WM microstructure in more detail ${ }^{4}$.

For the first time, a small, but statistically significant FA decline in the right cingulum over a 3-year time period was found in healthy siblings compared to controls. This earlier-than-expected by age WM decline in siblings without any (subclinical) psychotic symptoms may point to an aberrant WM development curvature and a potential WM intermediate phenotype. Replication of these results is needed, preferably including siblings at different age stages to acquire a more complete WM age-trajectory of this higher-than-average risk group.

The WM trajectory in the 3-year timeframe in patients with a psychotic disorder was rather stable, but appeared conditional on the level of cannabis use, the degree of childhood trauma experiences and exposure to antipsychotic (AP) medication. These findings provide evidence for an early neurodevelopmental cause of WM alterations in the pathology of psychotic disorder with a potential influence of (early) environ- 
mental factors and AP medication on WM development later in life. It is worthwhile to examine other environmental risk factors for psychotic disorder, such as pre-or perinatal birth defects, urban upbringing, and social exclusion, in relation to WM structure. This may help us to identify children at a young age with an increased risk of developing a psychotic disorder. Medical, psychological as well as societal precautions can then be taken to minimize the risk for psychosis as much as possible.

\section{Clinic: prevention, diagnostic and prognostic marker}

Diffusion tensor imaging has, despite numerous pitfalls ${ }^{5}$, proven to be a useful research tool for in vivo investigation of structural brain abnormalities in psychotic disorder. However, the above-mentioned findings are all based upon group differences and are not applicable to the individual patient in daily clinical practice. The presented data in this thesis may entail however promising new insights for daily and future clinical practice.

\section{Present tense}

(i) Although a clear causal relation between cannabis and trauma associated WM alterations over time and long-term prognosis in patients with psychotic disorder has not been established, physicians as well as patients should be aware of the potential impact of these environmental factors on cerebral vulnerability. This should not lead to negativism and determinism but to realistic information on resiliency and plasticity, thereby contributing to empowerment of patients and preventing professional stigmatization.

(ii) Society must be informed about adolescent cannabis use and childhood trauma as risk factors for the development of psychotic symptoms and the potential adverse effect on long-term WM brain development. Prevention campaigns should warn young adolescents not to experiment with or use cannabis, at least until their brains are fully matured. Evidence-based treatment programs for cannabis addiction (e.g. motivational interviewing) should be applied at an early phase in the treatment of first-episode patients with comorbid cannabis abuse.

(iii) Neighbours, primary school teachers, general practitioners and mental health workers should signal family problems at an earlier stage to prevent cases of childhood abuse. Youth services should be sufficiently funded to support dysfunctional families or placing children into foster families if necessary, to prevent potential childhood traumas. 


\section{(near) Future}

(iv) There is still a considerable diagnostic delay for the diagnosis of psychotic disorder, which has serious consequences for the patient and his recovery. Patients may drop out of school, alienate from their families and friends, become vulnerable for drug abuse, and have an increased suicide risk ${ }^{3}$. This delay is partly the result of the fact that patients with a psychiatric syndrome are diagnosed by thorough anamnesis and that in the prodromal or pre-psychotic phase the main symptoms can be vague and non-specific. An objective biological (imaging) marker is still missing, but would be very useful for diagnostic purposes, classification, staging of the disease, or prediction of the outcome ${ }^{6}$. The present thesis showed that longitudinal change in potential WM biomarkers might be relevant to further exploration, since the degree of exposure to an environmental stressor as well as the level of exposure to antipsychotic medication revealed different slopes of FA decline over time in patients with psychotic disorder. However, more specific and reliable WM measures are warranted in view of the disadvantages of the common applied diffusion indices (see below). Combining the clinical presentation of a patient with fixed interval WM measurements of specific regional WM tracts may provide us with information on the (positive or negative) effects of medication or drug use and finally, a long-term prognosis of the disease. However, whether a negative or positive WM slope is associated with worsening or amelioration of symptoms or a worse or better long-term outcome has not yet been established and needs further investigation.

(v) Given the overlapping FA findings in a various psychiatric disorders and the fact that diffusion data appears to be highly sensitive to common artifacts ${ }^{7}$, diffusion imaging falls short when applied for etiological, diagnostic and prognostic purposes in psychiatry. However, a network analysis in which the patients' symptoms, his functional and structural brain abnormalities, and his predisposed and current environmental stressors are combined in a mathematical algorithm, may be a more promising diagnostic tool in the near future ${ }^{8,9}$.

\section{Summary}

In conclusion, the presented results in this thesis have several implications, on a scientific level, as well as on clinical reasoning and intervention level. The longitudinal results on the WM course in healthy siblings and the childhood trauma and cannabis associated WM alterations in patients with a psychotic disorder warrant further replication. Understanding the timing and effect of environmental risk factors on neuronal brain matter is crucial, as well as the age-related trajectory of brain tissue. Whether it is static, worsens over time or ameliorates throughout a recovery process is essen- 
tial in the improvement of knowledge of psychosis outcome and for planning future therapeutic strategies. Therefore, these presented results should be disseminated through peer reviewed journals and presentations on national and international (schizophrenia) conferences, and also made accessible to patients and family members. Although the clinical implications of this work are hitherto limited, the presented results in this thesis may play a supporting role in the implementation of public prevention programs (through e.g. television, social media). The general public must be aware of the detrimental effects of childhood trauma exposure and adolescent cannabis use on the developing brain, resulting in an increased risk for psychiatric disorders. 


\section{References}

1. Jin H, Mosweu I. The Societal Cost of Schizophrenia: A Systematic Review. Pharmacoeconomics Aug 242016.

2. Millier A, Schmidt U, Angermeyer MC, Chauhan D, Murthy V, Toumi M, Cadi-Soussi N. Humanistic burden in schizophrenia: a literature review. Journal of psychiatric research Jul 2014;54:85-93.

3. Sommer IE, Bearden CE, van Dellen E, et al. Early interventions in risk groups for schizophrenia: what are we waiting for? NPJ Schizophr 2016;2:16003.

4. Karlsgodt KH. Diffusion Imaging of White Matter In Schizophrenia: Progress and Future Directions. Biol Psychiatry Cogn Neurosci Neuroimaging May 2016;1(3):209-217.

5. Jones DK, Knosche TR, Turner R. White matter integrity, fiber count, and other fallacies: the do's and don'ts of diffusion MRI. Neuroimage Jun 2013;73:239-254.

6. Linden DE. The challenges and promise of neuroimaging in psychiatry. Neuron Jan 12 2012;73(1):8-22.

7. Weinberger DR, Radulescu E. Finding the Elusive Psychiatric "Lesion" With 21st-Century Neuroanatomy: A Note of Caution. Am J Psychiatry Jan 2016;173(1):27-33.

8. Isvoranu AM, Borsboom D, van Os J, Guloksuz S. A Network Approach to Environmental Impact in Psychotic Disorder: Brief Theoretical Framework. Schizophr Bull Jul 2016;42(4):870-873.

9. van den Heuvel MP, Fornito A. Brain networks in schizophrenia. Neuropsychol Rev Mar $2014 ; 24(1): 32-48$. 

Summary

Samenvatting

\author{
Valorisation
}

Dankwoord

Curriculum Vitae

List of Publications 

"Weet je ... Ik vind het meestal gewoon leuk om dingen te maken die lekker klinken en lekker bekken zonder dat het ook maar iets om het lijf heeft. Weet je wel? Dat is al moeilijk genoeg. Ik doe er ook nooit langer dan twee dagen over. Als je er langer over doet dan twee dagen is het meestal gewoon shit, weet je wel. Het is geen relativiteitstheorie".

(Quote van Barry Hay in 'Donderweg - Mijn leven in de fast lane van de popmuziek' van Jaap Boots)

Het schrijven van dit proefschrift heeft ietsje langer geduurd dan twee dagen. Dat is je vast niet ontgaan. Maar het gaat dan ook niet om iets 'simpels' als het schrijven van het ultieme popliedje. Toch zie ik dit proefschrift ook wel een beetje als een eerste EP. Ooit onbesuisd eraan begonnen met haperende techniek (lees: software) en veel ongepolijst materiaal dat nog erg bijgeschaafd moest worden door de co-writers. De teksten barsten waarschijnlijk nog van de schoonheidsfoutjes en een echt eigen 'geluid' moet ik nog ontdekken, maar het (al niet meer) jeugdig enthousiasme en uithoudingsvermogen is er hopelijk uit af te lezen. Net zoals bij een EP is ook dit proefschrift slechts tot stand gekomen met veel hulp van belangrijke anderen. Dit productie- en schrijversteam, de band, techniek en uitzinnige groupies wil ik graag in min of meer willekeurige volgorde bedanken.

Special thanks to: Jim, Machteld, Ed, Stijn, Petra, Sanne, Christine, Truda, Inge, Wolfgang, alle proefpersonen, Alard, Vincent, Ron, Siemens, FSL, Maarten, Frank, Rob, Tineke, Nienke, Apple, Cécile, Philippe, Charlie, Manon, BrainVoyager, Trees, Johan, Dennis, Marjan, Lies, Koen, Debora, Kim, Rob, Marcel, Peter, Robert, Chris, Ritsaert, Irene, Mariëlle, Bart, Ruud, Gunter, Uli, Inez, Lydia, Ballorig, Richard, Rikus, Therese, Jolanda, Ine, Richel, Marijntje, Frans, Carsten, Albert, Jacqueline, Carla, Baer, Jan, Frenk, Ivo, Heidi, Freesurfer, Monique, Huib, Carin, Ingrid, Ruud, Harrie, Aafje, Nanda, Nina, HIC-team, K-Rock, Mia, Els, Herman, Loek, Vlieland, Benjamin, Min, René, Mondriaan, Spotify, Thomas, Jan Willem, Arno, David, Ivar, Pierre, Germaine, pap, mam, broertje, Ruben, Milan en mijn liefje...

... muito obrigado a todos!

Bij voorbaat mijn excuses aan de mensen die ik had moeten bedanken, maar vergeten ben in dit lijstje op te nemen. Oh, en die debuut LP laat nog even op zich wachten... 

Summary

Samenvatting

\author{
Valorisation
}

Dankwoord

\title{
Curriculum Vitae
}

\section{List of Publications}





\section{Curriculum Vitae}

Patrick Domen was born in Uden (the Netherlands) on December 20 ${ }^{\text {th }}, 1976$. In 1995 he graduated from secondary school (Atheneum) at the Mollerlyceum in Bergen op Zoom. Thereafter, he studied psychology at Maastricht University, which he substituted for medicine, after earning his first-year diploma in 1996. In 2002 he graduated at the Faculty of Medicine. Then, for seven months he worked as a resident psychiatry (not in training) at the department of addiction care at Mondriaan (Heerlen), after which he began his psychiatric training at the Universitaire Opleiding Psychiatrie in Maastricht (Vijverdal) in April 2003. Internships were followed at the closed ward, outpatient clinic for mood disorders, the psychiatric ward at the Maastricht University Medical Centre and the Riagg Maastricht. His last year was a combined internship of patient care in a Flexible-Assertive Community Treatment (F-ACT) team, a research project and teaching at the Faculty of Health, Medicine and Life sciences at Maastricht University. After completing the psychiatry training in October 2007, he started a PhD program at that same university, being also part-time seconded to Mondriaan, continuing his work in the F-ACT team. In April 2015 he replaced the F-ACT team for a position at the High \& Intensive Care (HIC) unit. Over the past years he fulfilled several teaching roles in the medicine curriculum and obtained the University Teaching Qualification (UTQ) certificate in 2013. As of April $1^{\text {st }} 2017$ he will be head of the Universitaire Opleiding Psychiatrie Zuid, Mondriaan.

$\mathrm{He}$ is married to Birgit and father of Ruben and Milan. 

Summary

Samenvatting

\author{
Valorisation
}

Dankwoord

Curriculum Vitae

List of Publications 



\section{Scientific publications in peer-reviewed journals}

Domen P, Peeters S, Michielse S, Gronenschild E, Viechtbauer W, Roebroeck A, Van Os J, Marcelis M for G.R.O.U.P. (2017). Differential Time Course of Microstructural White Matter in Patients With Psychotic Disorder and Individuals at Risk: A 3-Year Follow-up Study. Schizophrenia Bulletin, 43, 160-170.

Domen PA, Michielse S, Gronenschild E, Habets P, Roebroeck A, Schruers K, Van Os J, Marcelis M for G.R.O.U.P. (2013). Microstructural white matter alterations in psychotic disorder: A family-based diffusion tensor imaging study. Schizophrenia Research, 146, 291-300.

van der Leeuw C, Peeters S, Domen P, van Kroonenburgh M, van Os J, Marcelis M, Genetic Risk and Outcome in Psychosis (G.R.O.U.P.). (2015). Bone mineral density as a marker of cumulative estrogen exposure in psychotic disorder: A 3-year follow-up study. PLoS One, 26;10(8):e0136320.

van der Leeuw C, Habets P, Gronenschild E, Domen P, Michielse S, van Kroonenburgh M, van Os J, Marcelis M, for G.R.O.U.P. (2013). Testing the estrogen hypothesis of schizophrenia: associations between cumulative estrogen exposure and cerebral structural measures. Schizophrenia Research, 150, 114-20.

van der Leeuw $C$, Habets $P$, Domen $P$, van Kroonenburgh $M$, van Os J, Marcelis $M$, for G.R.O.U.P. (2013). Bone mineral density as a marker of cumulative endogenous estrogen exposure: relationship to background genetic risk of psychotic disorder. Schizophrenia Research, 143, 25-31.

\section{Book}

Bak M, Domen P, van Os (red). Innovatief leerboek Persoonlijke Psychiatrie. 2017. Leusden: Diagnosis uitgevers.

\section{Submitted for publication}

Domen P, Michielse S, Peeters S, Viechtbauer W, Van Os J, Marcelis M for G.R.O.U.P. (2017). Trauma- and cannabis-associated microstructural white matter changes in patients with psychotic disorder: A longitudinal family-based diffusion imaging study.

\section{Conference presentations}

Domen P, Michielse S, Gronenschild E, Habets P, Roebroeck A, Van Os J, Marcelis M for G.R.O.U.P. (2014). Witte stof veranderingen bij patienten met schizofrenie: de rol van omgevingsvariabelen. Voorjaarscongres NVvP, Maastricht.

Domen P, Peeters S, Michielse S, Gronenschild E, Van Os J, Marcelis M (2014). Reduced microstructural white matter alterations in psychotic disorder; stable or progressive? Schizophrenia Research, 153, S55-S56. 
Domen P, Gronenschild E, Michielse S, Habets P, Roebroeck A, Van Os J, Marcelis $M$ for G.R.O.U.P. (2013). Altered white matter integrity in schizophrenia: the role of environmental and familial risks. Schizophrenia Bulletin, 39, S180.

Domen P, Michielse S, Gronenschild E, Habets P, Roebroeck A, Van Os J, Marcelis M (2012). Microstructural white matter biomarkers for psychotic disorder: a familybased DTI study. Schizophrenia Research, 136, S203.

Marcelis M, Domen P, Michielse S, Gronenschild E, Habets P, Van Os J (2012). Decreased white matter integrity in psychotic disorder: association with childhood trauma? Schizophrenia Research, 136, S87-S88.

Domen P, Michielse S, Gronenschild E, Habets P, Roebroeck A, Schruers K, Van Os J, Marcelis M for G.R.O.U.P. (2012) Cerebrale dysconnectiviteit: biomarkers voor psychotische stoornis of indicator voor genetisch risico? Voorjaarscongres NVvP, Maastricht. Domen P. (2012) 'Cerebraal watermanagement'; met neuro-imaging op zoek naar de oorzaak van een psychotische stoornis. Zuid Limburgs Psychiater Genootschap. Maastricht. 